\title{
Capturing Missing Tuples and Missing Values
}

\author{
TING DENG, RCBD and SKLSDE, Beihang University \\ WENFEI FAN, Informatics, University of Edinburgh, and RCBD and SKLSDE, Beihang University \\ FLORIS GEERTS, Department of Mathematics and Computer Science, University of Antwerp
}

\begin{abstract}
Databases in real life are often neither entirely closed-world nor entirely open-world. Databases in an enterprise are typically partially closed, in which a part of the data is constrained by master data that contains complete information about the enterprise in certain aspects. It has been shown that, despite missing tuples, such a database may turn out to have complete information for answering a query.

This article studies partially closed databases from which both tuples and attribute values may be missing. We specify such a database in terms of conditional tables constrained by master data, referred to as $c$ instances. We first propose three models to characterize whether a $c$-instance $\mathcal{T}$ is complete for a query $Q$ relative to master data. That is, depending on how missing values in $\mathcal{T}$ are instantiated, the answer to $Q$ in $\mathcal{T}$ remains unchanged when new tuples are added. We then investigate three problems, to determine (a) whether a given $c$-instance is complete for a query $Q$, (b) whether there exists a $c$-instance that is complete for $Q$ relative to master data available, and (c) whether a $c$-instance is a minimal-size database that is complete for $Q$. We establish matching lower and upper bounds on these problems for queries expressed in a variety of languages in each of the three models for specifying relative completeness.
\end{abstract}

Categories and Subject Descriptors: H.2.3 [Database Management]: Languages

General Terms: Design, Algorithms, Theory

Additional Key Words and Phrases: Incomplete information, relative completeness, master data management, partially closed databases, complexity

ACM Reference Format:

Ting Deng, Wenfei Fan, and Floris Geerts. 2016. Capturing missing tuples and missing values. ACM Trans. Database Syst. 41, 2, Article 10 (May 2016), 47 pages.

DOI: http://dx.doi.org/10.1145/2901737

\section{INTRODUCTION}

Incomplete information has been a long-standing issue. The scale of the problem is such that it is common to find critical information missing from databases. For instance, it is estimated that pieces of information perceived as being needed for clinical decisions were missing $13.6 \%$ to $81 \%$ of the time [Miller Jr. et al. 2005]. Traditionally, the research community adopts either the Closed-World Assumption (CWA) or the Open-World Assumption (OWA). The CWA assumes that a database has collected

T. Deng and W. Fan are supported in part by grants 973 Program 2014CB340302 and NSFC 61421003. W. Fan is also supported in part by grants NSFC 61133002, 973 Program 2012CB316200, ERC 652976, EPSRC EP/J015377/1, and EP/M025268/1, NSF III 1302212, Shenzhen Peacock Program 1105100030834361, Guangdong Innovative Research Team Program 2011D005, Shenzhen Science and Technology Fund JCYJ20150529164656096, and Guangdong Applied R\&D Program 2015B010131006.

Authors' addresses: T. Deng, School of Computer Science and Engineering, Beihang University; W. Fan, School of Informatics, University of Edinburgh; F. Geerts, Department of Mathematics and Computer Science, University of Antwerp.

Permission to make digital or hard copies of part or all of this work for personal or classroom use is granted without fee provided that copies are not made or distributed for profit or commercial advantage and that copies show this notice on the first page or initial screen of a display along with the full citation. Copyrights for components of this work owned by others than ACM must be honored. Abstracting with credit is permitted. To copy otherwise, to republish, to post on servers, to redistribute to lists, or to use any component of this work in other works requires prior specific permission and/or a fee. Permissions may be requested from Publications Dept., ACM, Inc., 2 Penn Plaza, Suite 701, New York, NY 10121-0701 USA, fax +1 (212) 869-0481, or permissions@acm.org.

(c) 2016 ACM 0362-5915/2016/05-ART10 $\$ 15.00$

DOI: http://dx.doi.org/10.1145/2901737 
all the tuples representing real-world entities, but the values of some attributes in those tuples are possibly missing. The OWA assumes that some tuples representing real-world entities may also be missing (see Abiteboul et al. [1995] and van der Meyden [1998] for surveys).

Real-life databases are, however, often neither entirely closed-world nor entirely open-world. This is particularly evident in Master Data Management (MDM), one of the fastest growing software markets [Microsoft 2008; Radcliffe and White 2008]. Master data is a single repository of high-quality data that provides various applications with a synchronized, consistent view of the core business entities of an enterprise [Loshin 2008]. It is a closed-world database about the enterprise in certain aspects, for example, employees and customers. In the presence of master data, databases of the enterprise are typically partially closed [Fan and Geerts 2009, 2010b]. Whereas some parts of their data are constrained by the master data, for example, employees and customers, other parts of the databases are open-world, for example, sales transactions and service records.

Partially closed databases have recently been studied in Fan and Geerts [2009, $2010 \mathrm{~b}$, in the absence of missing values. Certain information in a partially closed database $\mathcal{I}$ is bounded by master data $D_{m}$, specified by a set $V$ of containment constraints $(\mathrm{CCs})$ from $\mathcal{I}$ to $D_{m}$. Relative to the master data $D_{m}$, the database $\mathcal{I}$ is then said to be complete for a query $Q$ if $Q(\mathcal{I})=Q\left(\mathcal{I}^{\prime}\right)$ for every partially closed extension $\mathcal{I}^{\prime}$ of $\mathcal{I}$, that is, for every $\mathcal{I}^{\prime}$ such that $\mathcal{I}^{\prime} \supsetneq \mathcal{I}$ and $\left(\mathcal{I}^{\prime}, D_{m}\right)$ satisfies $V$. That is, adding new tuples to $\mathcal{I}$ either does not change the query answer or violates the CCs. It is shown in Fan and Geerts [2009, 2010b] that, despite missing tuples, a partially closed database may still have complete information for answering queries.

The work of Fan and Geerts [2009, 2010b] has focused on ground instances, namely, database instances from which tuples are possibly missing, but all the values of the existing tuples are in place. In practice, however, both tuples and values are commonly found missing from a database. This introduces new challenges to characterizing and determining whether a database is complete for a query relative to master data.

Example 1.1. Let us first recall the setting in which only tuples may be missing from a database. Consider a database $D$ of uk patients, specified by the schema

\section{MVisit(NHS, name, city, yob, GD, Date, Diag, DrID),}

of which each tuple records the National Health Service (NHS) number (NHS), name, address (city), year of birthday (yob) and gender (GD) of a uK patient, as well as the date of visit to a doctor specified with ID (DrID) and the diagnosis given by the doctor. Consider a query $Q_{1}$ to find the names of those patients who were born in 2000 with NHS number '915-15-335' and live in Edinburgh. One can hardly trust the answer $Q_{1}(D)$ since tuples may be missing from $D$ even when no attribute values of the tuples in $D$ are missing.

Not all is lost. Suppose that there is master data $D_{m}$ available, specified by schema Patient $_{m}$ (NHS, name, yob, zip, GD), which provides a complete record of those patients living in Edinburgh and born after 1990. Then, we can conclude that $Q_{1}$ finds a complete answer in $D$ provided that $Q_{1}(D)$ returns all the patients $p$ in $D_{m}$ with $p$ [NHS]='915$15-335$ ' and $p[\mathrm{yob}]=2000$. In this case, there is no need to add new tuples to $D$ in order to find complete answers to query $Q_{1}$ in database $D$. Relative to master data $D_{m}$, the seemingly incomplete $D$ turns out to be complete for $Q_{1}$.

In practice, attribute values may also be missing. Following Grahne [1991] and Imieliński and Lipski Jr. [1984], we use a conditional table (c-table) $T$ to represent such a database, as shown in Figure 1 . In "tuple" $t_{2}$ of $T$, the values of $t_{2}$ [name] and $t_{2}$ [yob] are missing, and the condition $t_{2}$ [cond] tells us that $t_{2}$ [yob] is not 2001; similarly, the 


\begin{tabular}{|c|c|c|c|c|c|c|c|c|c|}
\cline { 2 - 9 } & NHS & name & city & yob & GD & Date & Diag & DrID & cond \\
$t_{1}:$ & $915-15-335$ & John & EDI & 2000 & M & $15 / 03 / 2015$ & Flu & 01 & \\
$t_{2}:$ & $915-15-356$ & $x$ & EDI & $z$ & F & $15 / 03 / 2015$ & Diabetes & 01 & $(z \neq 2001)$ \\
$t_{3}:$ & $915-15-357$ & Mary & $w$ & 2000 & F & $15 / 03 / 2015$ & Influenza & u & $(w \neq$ Edi $)$ \\
$t_{4}:$ & $915-15-358$ & Jack & LON & 2000 & M & $15 / 03 / 2015$ & Influenza & 02 & \\
$t_{5}:$ & $915-15-359$ & Louis & LON & 2000 & M & $15 / 03 / 2015$ & Diabetes & 03 & \\
\cline { 2 - 9 } & & &
\end{tabular}

Fig. 1. A $c$-table of a Patient.

condition $t_{3}$ [cond] tells us that $t_{3}$ [city] is not Edinburgh (Edi). Missing values introduce additional challenges. To characterize whether $T$ is complete for $Q_{1}$, we have to decide how to fill in the missing values in $T$ in addition to missing tuples.

These suggest that relatively complete databases have to accommodate not only missing tuples but also missing values. In addition, there are several fundamental questions that are not only of theoretical interest, but are also important to database users and developers. For instance, a user may be eager to know whether a database in use is complete for a query relative to master data. Furthermore, a developer may want to know what is a minimal amount of information that one has to collect to build a relatively complete database. These practical needs call for a full treatment of relative information completeness.

Relative information completeness. To capture missing values and missing tuples, we extend the notion of partially closed databases [Fan and Geerts 2009, 2010b] to $c$-instances. A $c$-instance is a collection of $c$-tables [Grahne 1991; Imieliński and Lipski Jr. 1984] in which certain parts are bounded by master data via a set of CCs [Fan and Geerts 2009] (see Section 2.1 for their formal definition).

Models. We propose three models to specify whether a $c$-instance $\mathcal{T}$ is complete for a query $Q$ relative to master data $D_{m}: \mathcal{T}$ is (1) strongly complete if each valuation of $\mathcal{T}$ yields a ground instance that is complete for $Q$ relative to $D_{m} ;(2)$ weakly complete if one can find in $\mathcal{T}$ the certain answers to $Q$ over all partially closed extensions of valuations of $\mathcal{T}$; and (3) viably complete if there exists a valuation of $\mathcal{T}$ that is a relatively complete database for $Q$. A user may choose a model that best serves one's needs.

Data consistency. We are interested in databases that are both relatively complete and consistent. The consistency of data is typically specified by integrity constraints, such that errors and conflicts in the data can be detected as violations of the constraints [Arenas et al. 1999; Chomicki 2007] (see Fan and Geerts [2012] for a recent survey). We investigate the impact of integrity constraints on the analysis of relative completeness. In addition, instead of using a separate language of integrity constraints, we adopt a class of CCs that is also capable of expressing constraints commonly used in data cleaning. More specifically, we consider CCs that can be expressed in terms of conjunctive queries.

Analysis of $c$-instances. We provide complexity bounds on basic issues in connection with $c$-instances. These problems are to decide, given a $c$-instance $\mathcal{T}$, whether $\mathcal{T}$ is (a) consistent, that is, whether there is any partially closed database represented by $\mathcal{T}$; and (b) extensible, that is, whether there exists any partially closed extension of $\mathcal{T}$.

Main complexity results. We identify three fundamental problems associated with relative information completeness. Given a query $Q$ and master data $D_{m}$,

- the relatively complete database problem (denoted by RCDP) is to decide whether a given database is complete for $Q$ relative to $D_{m}$; 
Table I. Complexity Results in Connection with Relative Completeness

\begin{tabular}{|c|c|c|c|}
\hline $\mathcal{L}_{Q}$ & RCDP & RCQP & MINP \\
\hline Strong completeness & Theorem 4.1 & Corollary 4.5 & Theorem 4.8 \\
\hline $\begin{array}{c}\mathrm{FO}, \mathrm{FP} \\
\mathrm{UCQ}, \exists \mathrm{FO}^{+}, \mathrm{CQ}\end{array}$ & $\begin{array}{l}\text { undecidable } \\
\Pi_{2}^{p} \text {-complete }\end{array}$ & $\begin{array}{l}\text { undecidable } \\
\text { NEXPTIME-c }\end{array}$ & $\begin{array}{c}\text { undecidable } \\
\Pi_{3}^{p} \text {-complete }\left(\mathrm{D}_{2}^{p} \text {-c) }\right.\end{array}$ \\
\hline Weak completeness & Theorem 5.1 & Theorem 5.4 & Theorem 5.6 \\
\hline $\begin{array}{c}\mathrm{FO} \\
\mathrm{FP} \\
\mathrm{UCQ}^{\exists}, \exists \mathrm{FO}^{+} \\
\mathrm{CQ}\end{array}$ & $\begin{array}{l}\text { undecidable } \\
\text { coneXPTIME-c } \\
\Pi_{3}^{p} \text {-complete } \\
\Pi_{3}^{p} \text {-complete }\end{array}$ & $\begin{array}{c}\text { ? (undecidable) } \\
O(1) \\
O(1) \\
O(1)\end{array}$ & $\begin{array}{c}\text { undecidable } \\
\text { conEXPTIME-c } \\
\Pi_{4}^{p} \text {-complete } \\
\text { coDP-complete }\end{array}$ \\
\hline Viable completeness & Theorem 6.1 & Corollary 6.2 & Corollary 6.3 \\
\hline $\begin{array}{c}\mathrm{FO}, \mathrm{FP} \\
\mathrm{UCQ}, \exists \mathrm{FO}^{+}, \mathrm{CQ}\end{array}$ & $\begin{array}{c}\text { undecidable } \\
\Sigma_{3}^{p} \text {-complete }\left(\Pi_{2}^{p} \text {-c) }\right.\end{array}$ & $\begin{array}{l}\text { undecidable } \\
\text { NEXPTIME-c }\end{array}$ & $\begin{array}{c}\text { undecidable } \\
\Sigma_{3}^{p} \text {-complete }\left(\mathrm{D}_{2}^{p} \text {-c) }\right.\end{array}$ \\
\hline
\end{tabular}

Note: Here, NeXPTIME-c, coneXPTIME-c, $D_{2}^{p}$-c and $\Pi_{2}^{p}$-c are abbreviations for NEXPTIMEcomplete, coNEXPTIME-complete, $\mathrm{D}_{2}^{p}$-complete and $\Pi_{2}^{p}$-complete, respectively.

- the relatively complete query problem (RCQP) asks whether it is possible to build a database complete for $Q$ relative to $D_{m}$; and

-the minimality problem (MINP) is to determine whether a database has a minimal size among those that are complete for $Q$ relative to $D_{m}$.

We investigate these problems with regard to several parameters:

$-\mathcal{L}_{Q}$ : the query language in which $Q$ is expressed, ranging over conjunctive queries, $(\mathrm{CQ})$, union of conjunctive queries (UCQ), positive existential $\mathrm{FO}$ queries $\left(\exists \mathrm{FO}^{+}\right)$, first-order queries (FO), and $\mathrm{FP}$, an extension of $\exists \mathrm{FO}^{+}$with an inflational fix-point operator;

- $c$-instances versus ground instances, that is, in the presence or absence of missing values; and

—different models of relative completeness, that is when a $c$-instance is required to be strongly complete, weakly complete, or viably complete for $Q$, relative to $D_{m}$ and $V$.

All these languages allow equality $(=)$ and inequality $(\neq)$, as supported by commercial DBMS; moreover, with $\neq$, we can express CCs and queries in the same query language (see Section 2.1 for CCs).

We provide a comprehensive picture of these problems with different combinations of these parameters. We establish their lower and upper bounds, all matching, ranging over $O(1)$, coDP, $\Pi_{2}^{p}, \Sigma_{2}^{p}, \mathrm{D}_{2}^{p}, \Pi_{3}^{p}, \Sigma_{3}^{p}, \Pi_{4}^{p}$, NEXPTIME, conEXPTIME, and undecidable. We summarize the main complexity results in Table I, in which the complexity bounds for ground instances are also listed (enclosed in parentheses) when they differ from their counterparts for $c$-instances, annotated with their corresponding theorems. In addition, we identify tractable special cases of these problems (Section 7).

Our main conclusions are as follows.

(a) All problems are decidable for $\mathrm{CQ}, \mathrm{UCQ}$, and $\exists \mathrm{FO}^{+}$, but are mostly undecidable for FO and FP. However, they are decidable for FP in the weak completeness model. Moreover, some problems for CQ and UCQ exhibit different behaviors.

(b) The presence of missing values makes our lives harder when RCDP and MINP are concerned. For example, in the strong completeness model, MINP for CQ is $\mathrm{D}_{2}^{p}$-complete for ground instances while it is $\Pi_{3}^{p}$-complete for $c$-instances; in the viable completeness 
model, RCDP for $\mathrm{CQ}$ is $\Pi_{2}^{p}$-complete for ground instances while it is $\Sigma_{3}^{p}$-complete for $c$-instances. In contrast, it does not complicate the analyses of RCQP. That is, the complexity of RCDP remains the same for ground or $c$-instances.

(c) The problems have rather diverse complexities in the three different models of relative completeness. For instance, RCQP for FP is undecidable in the strong completeness model, but is trivially decidable for weakly complete $c$-instances. Moreover, in the strong completeness model, RCQP for $c$-instances is equivalent to RCQP for ground instances, but this is no longer the case in the weak completeness model: the undecidability of RCQP for FO for ground instances cannot show the undecidability for $c$-instances (see Example 5.3; the precise complexity bounds of RCQP for FO and $c$ instances remain an open problem). On the other hand, RCDP for UCQ is $\Pi_{2}^{p}$-complete for the strongly complete $c$-instances, but it becomes $\Pi_{3}^{p}$-complete in the weak model.

(d) Master data and CCs do not substantially complicate the analyses of these problems. From the proofs given in Sections 4 to 6 , we can see that all lower bounds of RCDP, RCQP, and MINP hold when master data and CCs are fixed, except for RCDP $(C Q)$ and $\operatorname{MINP}(C Q)$ in the weak completeness model.

To the best of our knowledge, apart from the conference version of this article [Fan and Geerts 2010a], this work is a first treatment of relatively complete databases in the presence of both missing values and missing tuples. We identify important problems associated with partially closed $c$-instances, and provide matching complexity bounds on these problems. A variety of techniques are used to prove these results, including finite-model theoretic constructions, characterizations of relatively complete databases, and a wide range of reductions.

Related work. This work extends Fan and Geerts [2009] and Fan and Geerts [2010b] by dealing with missing tuples and missing values. We propose three models for relatively complete $c$-instances, which were not considered in these earlier works. For ground instances in the strong model, RCDP and RCQP have been studied in Fan and Geerts [2009, 2010b] with several cases left open there, but neither $c$-instances nor ground instances and $c$-instances for MINP have been considered there. The data complexity of RCDP and MINP for ground instances has been studied in Cao et al. [2014]. While we mostly focus on combined complexity in this article, we identify tractable cases of the three problems for data complexity.

This work is an extended version of the conference version [Fan and Geerts 2010a] by including the detailed proofs of all results, which were not presented in Fan and Geerts [2010a], and a variety of tractable cases (data complexity) in Section 7. To keep the article within a reasonable page limit, we do not consider the boundedness problem, which is studied in Fan and Geerts [2010a]. Moreover, we set the record straight by providing correct lower and upper bounds: (a) in the strong completeness model, $\operatorname{RCDP}\left(\mathcal{L}_{Q}\right)$ for $\mathrm{CQ}$ is $\Pi_{2}^{p}$-complete instead of $\Pi_{3}^{p}$-complete for $c$-instances (see Theorem 4.1); and (b) in the strong completeness or viable completeness model, $\operatorname{MINP}\left(\mathcal{L}_{Q}\right)$ for $\mathrm{CQ}$ is $\mathrm{D}_{2}^{p}$-complete instead of $\Delta_{3}^{p}$-complete for ground instances (see Theorem 4.8).

There has been a host of work on incomplete information, notably, representation systems (see Abiteboul et al. [1995] and van der Meyden [1998] for surveys, and more recently, Olteanu et al. [2008]). This work adopts c-tables [Grahne 1991; Imieliński and Lipski Jr. 1984] to represent databases with missing values. Our weak model for relative completeness is based on the certain answer semantics [Imieliński and Lipski Jr. 1984], and the strong model has a resemblance to strong representation systems. In contrast, viably complete $c$-instances do not find a counterpart in Grahne [1991] and Imieliński and Lipski Jr. [1984]. The basic issues for $c$-instances (see Section 3) are similar to the problems studied in Abiteboul et al. [1991], but in the presence of master 
data. As opposed to prior work in this area, we aim to model partially closed databases as found in MDM, and to settle their associated decision problems that have not been studied before.

Several approaches have been proposed for modeling databases with missing tuples (e.g., Gottlob and Zicari [1988], Levy [1996], Motro [1989], and Vardi [1986]). A notion of open null was introduced in Gottlob and Zicari [1988] to model locally controlled openworld databases, in which tuples or values can be marked with open null, while the rest of the data is closed-world. Complete and consistent extensions of an incomplete database were studied in Vardi [1986]. There has also been work on modeling negative information via logic programming (see van der Meyden [1998]). Neither master data nor the decision problems studied in this work have been considered there.

Closer to this work are partially complete databases studied in Levy [1996] and Motro [1989], which assume a virtual database $D_{c}$ that contains complete information in all relevant aspects, and assume that any database $D$ either contains or is defined as views of $D_{c}$. A notion of answer completeness was proposed there to decide whether a query posed on $D_{c}$ can be answered in $D$. We assume neither the existence of $D_{c}$ with entire complete information nor views that define $D$ in terms of $D_{c}$. In addition, neither missing values nor the problems studied here were considered in Levy [1996] and Motro [1989].

Certain answers have also been studied in data integration and data exchange. In data integration, for a query $Q$ posed on a global database $D_{G}$, one wants to find the certain answers to $Q$ over all data sources that are consistent with $D_{G}$ with regard to view definitions (e.g., see Abiteboul and Duschka [1998] and Lenzerini [2002]). In data exchange, one wants to find the certain answers to a query over all target databases transformed from data sources via schema mapping (see Kolaitis [2005] and Arenas et al. [2009]). The decision problems studied here are not considered in data exchange or data integration. There has also been work on answering queries using views to decide, for example, whether views determine queries [Segoufin and Vianu 2005]. Our decision problems cannot be reduced to the problems studied there, and vice versa, because in MDM, one often cannot characterize databases as views of master data.

There has also been work on consistent query answering (e.g., Arenas et al. [1999] and Chomicki [2007]) to find certain answers to a query over all repairs of a database. Master data is not considered there, and we do not consider database repairs in this work. For ground instances in the strong model, RCDP is similar to the problem of query independence from updates [Elkan 1990; Levy and Sagiv 1993]. However, none of the results of Elkan [1990] and Levy and Sagiv [1993] carries over to our setting. We refer to Fan and Geerts [2009, 2010b] for a more detailed discussion of related work on RCDP and RCQP for ground instances.

Related to this work is that of Libkin [2014], which proposes a new interpretation of query answers over incomplete data. It treats incomplete databases as logical theories, and query answering as logical implication (rather than certain answers); it defines representation systems under the CWA and OWA with respect to an information ordering. In contrast to Libkin [2014], we study relative information completeness in the presence of master data for databases that are neither entirely closed-world not entirely open-world. In this setting, we define three completeness models (strong, weak, and viable), and investigate associated problems RCDP, RCQP, and MINP for deciding relative completeness, which are not considered in Libkin [2014]. Note that the models of completeness and the decision problems studied here are also meaningful under the new semantics of Libkin [2014], although the complexity bounds may be different.

Complementary to this work is the recent work on assessing partial results, that is, query answers computed with incomplete input due to failures in data access [Lang 
et al. 2014]. With respect to incomplete data sources, it proposes a framework to classify partial results (i.e., cardinality and correctness) and to determine the degree of partial result classification precision. In contrast, we study how to determine whether input data is complete for our queries relative to available master data. The problems studied in this work are not considered in Lang et al. [2014], and vice versa. That said, after the input is found incomplete, the methods of Lang et al. [2014] can be triggered to evaluate the quality of partial answers computed from the input.

Organization. Section 2 presents three models for specifying relatively complete $c$-instances. Section 3 investigates the impact of integrity constraints and basic issues in connection with $c$-instances. Problems RCDP, RCQP, and MINP are studied in Sections 4, 5, and 6 for strongly complete, weakly complete, and viably complete $c$ instances, respectively. Section 7 identifies special cases with tractable data complexity. Section 8 summarizes the main results and identifies open problems.

\section{RELATIVE INFORMATION COMPLETENESS REVISITED}

In Section 2.1, we first review relatively complete ground instances defined in Fan and Geerts $[2009,2010 \mathrm{~b}]$. In Section 2.2, we present three models to characterize relatively complete $c$-instances. Finally, in Section 2.3, we state the decision problems associated with relative information completeness.

\subsection{Relatively Complete Ground Instances}

A database schema $\mathcal{R}$ is a collection $\left(R_{1}, \ldots, R_{n}\right)$ of relation schemas. Each $R_{i}$ is defined over a set of attributes. Its set of attributes is also denoted by $R_{i}$. For each attribute $A$ in $R_{i}$, its finite or infinite domain is a set of constants, denoted by $\operatorname{dom}(A)$.

Ground instances and master data. A ground instance $\mathcal{I}$ of $\mathcal{R}$ is of the form $\left(I_{1}, \ldots, I_{n}\right)$, where for each $i \in[1, n], I_{i}$ is an instance of $R_{i}$ without missing values. That is, for each $t \in I_{i}$ and each $A \in R_{i}, t[A]$ is a constant in $\operatorname{dom}(A)$.

Master data $D_{m}$ is a ground instance of a database schema $\mathcal{R}_{m}$. It is a consistent and closed-world database.

Partially closed databases. We specify the relationship between a database and master data in terms of CCs. A CC $\phi$ is of the form $q(\mathcal{R}) \subseteq p\left(\mathcal{R}_{m}\right)$, where $q$ is a conjunctive query (CQ) defined over schema $\mathcal{R}$, and $p$ is a projection query over schema $\mathcal{R}_{m}$. A ground instance $\mathcal{I}$ of $\mathcal{R}$ and master data $D_{m}$ of $\mathcal{R}_{m}$ satisfy $\phi$, denoted by $\left(\mathcal{I}, D_{m}\right) \models$ $\phi$, if $q(\mathcal{I}) \subseteq p\left(D_{m}\right)$.

Intuitively, the CWA is asserted for $D_{m}$, which imposes an upper bound on the information extracted by $q(\mathcal{I})$ from the database $\mathcal{I}$. On the other hand, the OWA is assumed on the part of $\mathcal{I}$ that is not constrained by CCs.

Example 2.1. Recall the database $D$ and master data $D_{m}$ described in Example 1.1. We specify a set $V$ of CCs such that, for each year $y$ in the range [1991, 2014], $V$ includes the CC $q_{y}$ (MVisit) $\subseteq p$ (Patient $\left.{ }_{m}\right)$, where $q_{y}(n, n a, y, g)=$ $\exists d, d i, i$ (MVisit $(n, n a, c, y, g, d, d i, i) \wedge c=' \mathrm{EDI})$, and $p\left(n, n a, y^{\prime}, g\right)=\exists z\left(\operatorname{Patient}_{m}(n\right.$, $\left.n a, y^{\prime}, z, g\right)$ ), which ensures that $D_{m}$ is an upper bound on the information in $D$ about patients who live in Edinburgh and are born between 1991 and 2014.

Certain integrity constraints can also be expressed as CCs. For example, consider a functional dependency (FD) $\phi:(\mathrm{NHS} \rightarrow$ name, GD), which specifies that, in the UK, the NHS number determines the name and gender of each patient. Furthermore, assume that master data contains an empty relation $D_{\emptyset}$. Then, the FD $\phi$ can be enforced by 
including the following two CCs in $V: q_{\text {name }} \subseteq D_{\emptyset}$ and $q_{\mathrm{GD}} \subseteq D_{\emptyset}$, where

$q_{\text {name }}=\exists n, n a_{1}, n a_{2}, c_{1}, c_{2}, y_{1}, y_{2}, g_{1}, g_{2}, d_{1}, d_{2}, d i_{1}, d i_{1}, i_{1}, i_{2}$

$\left(\operatorname{MVisit}\left(n, n a_{1}, c_{1}, y_{1}, g_{1}, d_{1}, d i_{1}, i_{1}\right) \wedge \operatorname{MVisit}\left(n, n a_{2}, c_{2}, y_{2}, g_{2}, d_{2}, d i_{2}, i_{2}\right) \wedge n_{1} \neq n_{2}\right)$,

which detects violations of the FD NHS $\rightarrow$ name; similarly, $q_{\mathrm{GD}}$ is defined to detect violations of the FD NHS $\rightarrow$ GD. Note that we allow inequalities in $\mathrm{CQ}$, hence also in CCs. It is shown in Fan and Geerts [2009, 2010b] that inclusion dependencies (INDs) can be expressed as $\operatorname{CCs} q(\mathcal{R}) \subseteq p\left(\mathcal{R}_{m}\right)$ when $q$ is in FO, referred to as CCs in FO (see more details about INDs in Section 3 ).

We say that $\left(\mathcal{I}, D_{m}\right)$ satisfies a set $V$ of CCs, denoted by $\left(\mathcal{I}, D_{m}\right) \models V$, if $\left(\mathcal{I}, D_{m}\right) \models \phi$ for each CC $\phi$ in $V$.

A ground instance $\mathcal{I}$ of $\mathcal{R}$ is said to be partially closed relative to $\left(D_{m}, V\right)$ if $\left(\mathcal{I}, D_{m}\right) \models$ $V$. That is, the information in $\mathcal{I}$ is partially bounded by $D_{m}$ via the CCs in $V$.

Relatively complete ground instances. Consider ground instances $\mathcal{I}=\left(I_{1}, \ldots, I_{n}\right)$ and $\mathcal{I}^{\prime}=\left(I_{1}^{\prime}, \ldots, I_{n}^{\prime}\right)$ of $\mathcal{R}$. We say that the instance $\mathcal{I}^{\prime}$ extends $\mathcal{I}$, denoted by $\mathcal{I} \subsetneq \mathcal{I}^{\prime}$, if for all $i \in[1, n], I_{i} \subseteq I_{i}^{\prime}$, and furthermore, there is a $j \in[1, n]$ such that $I_{j} \subsetneq I_{j}^{\prime}$. The set of partially closed extensions of $\mathcal{I}$ is defined as

$$
\operatorname{Ext}\left(\mathcal{I}, D_{m}, V\right)=\left\{\mathcal{I}^{\prime} \mid \mathcal{I} \subsetneq \mathcal{I}^{\prime},\left(\mathcal{I}^{\prime}, D_{m}\right) \models V\right\}
$$

That is, for each $\mathcal{I}^{\prime}$ in the set, (a) $\mathcal{I}^{\prime}$ extends $\mathcal{I}$ by including new tuples, and (b) $\mathcal{I}^{\prime}$ is partially closed relative to $\left(D_{m}, V\right)$. We write $\operatorname{Ext}\left(\mathcal{I}, D_{m}, V\right)$ as $\operatorname{Ext}(\mathcal{I})$ when $D_{m}$ and $V$ are clear from the context.

A ground instance $\mathcal{I}$ is said to be complete for a query $Q$ relative to $\left(D_{m}, V\right)$ if (i) it is partially closed; and (ii) for each $\mathcal{I}^{\prime} \in \operatorname{Ext}(\mathcal{I}), Q(\mathcal{I})=Q\left(\mathcal{I}^{\prime}\right)$. In other words, the answer to $Q$ in $\mathcal{I}$ remains unchanged no matter what new tuples are added to $\mathcal{I}$. Intuitively, $\mathcal{I}$ already has complete information for answering $Q$. The completeness is relative to $\left(D_{m}, V\right)$ : the extensions must satisfy $V$.

Example 2.2. Recall the ground instances $D, D_{m}$, and the query $Q_{1}$ from Example 1.1, and let $V$ be the set of CCs from Example 2.1. Then, as shown in Example 1.1, $D$ is complete for $Q_{1}$ relative to $\left(D_{m}, V\right)$ as long as it returns all relevant tuples in $D_{m}$.

Consider another query $Q_{2}$, which is to find the names of all patients who were born in 2000 and have NHS number 915-15-321. Suppose that there are such patient records in $D_{m}$, but $Q_{2}(D)$ is empty. Then, $D$ is not complete for $Q_{2}$. We can make $D$ complete for $Q_{2}$, however, by adding to $D$ a single tuple $t$ with $t$ [NHS] $=$ '915-15-321'. $V$ includes the CCs encoding FD $\phi$, which ensures that there exists at most one patient with this NHS number. Thus, the extended $D$ is complete for $Q_{2}$ relative to $\left(D_{m}, V\right)$.

In contrast, consider the query $Q_{3}$, which is to find the names of all patients who were diagnosed as diabetics in 2000 , no matter where they live. Then, the master data $D_{m}$ does not help. It has no information about patients living in cities other than Edinburgh. In this case, we cannot make $D$ complete for $Q_{3}$ relative to $\left(D_{m}, V\right)$.

\subsection{Accommodating Missing Values}

To specify databases with missing values, we adopt conditional tables (c-tables) that are specified using variables and local conditions [Grahne 1991; Imieliński and Lipski Jr. 1984]. To define $c$-tables, for each relation schema $R_{i}$ and each attribute $A$ in $R_{i}$, we assume a countably infinite set $\operatorname{var}(A)$ of variables such that $\operatorname{var}(A) \cap \operatorname{dom}(A)=\emptyset$, $\operatorname{var}(A) \cap \operatorname{dom}(B)=\emptyset$, and $\operatorname{var}(A) \cap \operatorname{var}(B)=\emptyset$ for every attribute $B$ distinct from $A$. 
Partially closed $c$-instances. A $c$-table of $R_{i}$ is a pair $(T, \xi)$, where (a) $T$ is a tableau in which for each tuple $t$ and each attribute $A$ in $R_{i}, t[A]$ is either a constant in $\operatorname{dom}(A)$ or a variable in $\operatorname{var}(A)$; and (b) $\xi$ is a mapping that associates a condition $\xi(t)$ with each tuple $t$ in $T$. Here, $\xi(t)$ is built up from atoms $x=y, x \neq y, x=c, x \neq c$, by closing under conjunction $\wedge$, where $x, y$ are variables and $c$ is a constant. Denote by $(T$, true) the $c$-table without any conditions. An example of a $c$-table is shown in Figure 1.

A valuation $\mu$ of $(T, \xi)$ is a mapping such that, for each tuple $t$ in $T$ and each attribute $A$ in $R_{i}, \mu(t[A])$ is a constant in $\operatorname{dom}(A)$ if $t[A]$ is a variable, and $\mu(t[A])=t[A]$ if $t[A]$ is a constant. Let $\mu(t)$ be the tuple of $R_{i}$ obtained by substituting $\mu(x)$ for each occurrence of $x$ in $t$. Then, we define

$$
\mu(T)=\{\mu(t) \mid t \in T \text { and } \xi(\mu(t)) \text { evaluates to true }\} .
$$

Hence, $\mu(T)$ is a ground instance without variables or conditions. More specifically, $(T, \xi)$ represents a set of possible worlds $\mu(T)$ when $\mu$ ranges over all valuations of $(T, \xi)$. We write $(T, \xi)$ simply as $T$ when $\xi$ is clear from the context.

A $c$-instance $\mathcal{T}$ of $\mathcal{R}$ is of the form $\left(T_{1}, \ldots, T_{n}\right)$, where for each $i \in[1, n], T_{i}$ is a $c$-table of $R_{i}$. A valuation $\mu$ of $\mathcal{T}$ is of the form $\left(\mu_{1}, \ldots, \mu_{n}\right)$, where $\mu_{i}$ is a valuation of $T_{i}$. We use $\mu(\mathcal{T})$ to denote the ground instance $\left(\mu_{1}\left(T_{1}\right), \ldots, \mu_{n}\left(T_{n}\right)\right)$ of $\mathcal{R}$. A partially closed $c$-instance $\mathcal{T}$ represents a nonempty set of partially closed ground instances, denoted by $\operatorname{Mod}\left(\mathcal{T}, D_{m}, V\right)$. That is,

$$
\operatorname{Mod}\left(\mathcal{T}, D_{m}, V\right)=\left\{\mu(\mathcal{T}) \mid \mu \text { is a valuation and }\left(\mu(\mathcal{T}), D_{m}\right) \models V\right\}
$$

We write $\operatorname{Mod}\left(\mathcal{T}, D_{m}, V\right)$ as $\operatorname{Mod}(\mathcal{T})$ when $D_{m}$ and $V$ are clear from the context. We say that a $c$-instance $\mathcal{T}$ is partially closed if $\operatorname{Mod}\left(\mathcal{T}, D_{m}, V\right)$ is not empty.

To simplify the discussion, in the sequel, we consider only $c$-instance $\mathcal{T}$ for which $\operatorname{Mod}(\mathcal{T})$ is nonempty. The assumption has no impact on the complexity results of this article. As will be shown by Proposition 3.3 , it is in $\Sigma_{2}^{p}$ to decide whether $\operatorname{Mod}(\mathcal{T})$ is nonempty. As we can see from Table I, all the complexity bounds of this article are higher than $\Sigma_{2}^{p}$-complete except RCDP for $\mathrm{CQ}, \mathrm{UCQ}$, and $\exists \mathrm{FO}^{+}$in the strong completeness model, and MINP(CQ) in the weak completeness model. For these two problems, we will show that their complexity bounds remain intact without the assumption.

Databases under the CWA or the OWA are special cases of partially closed $c$ instances. Recall that the CWA assumes that a database has collected all the tuples representing real-world entities, but the values of some attributes in those tuples are possibly missing; the OWA assumes that some tuples representing real-world entities may also be missing. Thus, a $c$-instance $\mathcal{T}$ is open-world in the absence of master data and CCs and closed-world if the master data is a possible world represented by $\mathcal{T}$.

Relative completeness. We next define various notions of completeness for $c$ instances. We say that, relative to $\left(D_{m}, V\right)$, a partially closed $c$-instance $\mathcal{T}$ is

-strongly complete for $Q$ if for each $\mathcal{I} \in \operatorname{Mod}(\mathcal{I})$ and for each $\mathcal{I}^{\prime} \in \operatorname{Ext}(\mathcal{I}), Q(\mathcal{I})=Q\left(\mathcal{I}^{\prime}\right)$; -weakly complete for $Q$ if

$$
\bigcap_{\mathcal{I} \in \operatorname{Mod}(\mathcal{I})} Q(\mathcal{I})=\bigcap_{\mathcal{I} \in \operatorname{Mod}(\mathcal{T}), \mathcal{I}^{\prime} \in \operatorname{Ext}(\mathcal{I})} Q\left(\mathcal{I}^{\prime}\right),
$$

or for all $\mathcal{I} \in \operatorname{Mod}(\mathcal{T}), \operatorname{Ext}(\mathcal{I})=\emptyset$; and

-viably complete for $Q$ if there exists $\mathcal{I} \in \operatorname{Mod}(\mathcal{T})$ such that for each $\mathcal{I}^{\prime} \in \operatorname{Ext}(\mathcal{I})$, $Q(\mathcal{I})=Q\left(\mathcal{I}^{\prime}\right)$.

Intuitively, (a) $\mathcal{T}$ is strongly complete if, no matter how missing values in $\mathcal{T}$ are filled in, it yields a ground instance relatively complete for $Q$; (b) $\mathcal{T}$ is weakly complete if the certain answer to $Q$ over all partially closed extensions of $\mathcal{T}$ can already be found in 
$\mathcal{T}$; and (c) $\mathcal{T}$ is viably complete if there exists a way to instantiate missing values in $\mathcal{T}$ that results in a ground instance relatively complete for $Q$.

We use $\operatorname{RCQ}^{s}\left(Q, D_{m}, V\right), \mathrm{RCQ}^{w}\left(Q, D_{m}, V\right)$, and $\operatorname{RCQ}^{v}\left(Q, D_{m}, V\right)$ to denote the set of all complete $c$-instances of $\mathcal{R}$ for $Q$ with regard to $\left(D_{m}, V\right)$, in the strong, weak, and viable completeness models, respectively. We simply use $\operatorname{RCQ}\left(Q, D_{m}, V\right)$ when there is no need to distinguish the completeness models.

Example 2.3. Consider the $c$-instance $T$ shown in Figure 1, master data $D_{m}$ and query $Q_{1}$ of Example 1.1, and the set $V$ of CCs of Example 2.1. Then, $T$ is strongly complete for $Q_{1}$ relative to $\left(D_{m}, V\right)$. By the FD $\phi$ encoded as CCs in $V$, we have that, for all valuations $\mu$ of $T, Q_{1}(\mu(T))$ returns a single tuple (name='John'), and the answer to $Q_{1}$ does not change for every partially closed extension in $\operatorname{Ext}(\mu(T))$.

Consider query $Q_{4}$ to find the names of patients in Edinburgh who are born in 2000 and visited doctors on 15/03/2015. Suppose that $t_{m}^{1}$ and $t_{m}^{2}$ are the only patients in $D_{m}$ born in 2000, where $t_{m}^{1}=\left(915-15-335\right.$, John, M, EH8 9AB, 2000) and $t_{m}^{2}=(915-15-336$, Bob, M, EH8 9AB, 2000). Then, relative to $\left(D_{m}, V\right), T$ is viably complete for $Q_{4}$, since there exists a valuation $\mu$ of $T$ such that $\mu(T)$ is complete. For instance, this happens for $\mu(x)=$ Bob and $\mu(z)=2000$. The $c$-instance $T$ is also weakly complete, since the certain answer (name $=$ 'John') can already be found over $\operatorname{Mod}(T)$. However, $T$ is not strongly complete for $Q_{4}$. Consider $\mu^{\prime}(T)$ with $\mu^{\prime}(x)=$ John and $\mu^{\prime}(z)=2000$, and $\mu(T)$ defined as before. Then, clearly, $\mu^{\prime}(T) \subseteq \mu(T)$; moreover, $Q_{4}\left(\mu^{\prime}(T)\right)$ only returns John, whereas $Q_{4}(\mu(T))$ returns both John and Bob.

We observe the following: (a) If $\mathcal{T}$ is strongly complete, then it is both weakly complete and viably complete. (b) A ground instance $\mathcal{I}$ is a $c$-instance without variables and conditions. It is strongly complete and viably complete for a query $Q$ if and only if $\mathcal{I}$ is relatively complete for $Q$, as defined in Section 2.1 . However, $\mathcal{I}$ may be weakly complete but not relatively complete.

Minimal complete databases. To decide what data should be collected in a database to answer a query $Q$, we want to identify a minimal amount of information that is complete for $Q$. For this, we use the following notions of minimality.

A ground instance $\mathcal{I}$ is a minimal instance complete for a query $Q$ relative to $\left(D_{m}, V\right)$ if it is in $\operatorname{RCQ}\left(Q, D_{m}, V\right)$. Moreover, for all $\mathcal{I}^{\prime} \subsetneq \mathcal{I}$, we have that $\mathcal{I}^{\prime}$ is not in $\operatorname{RCQ}\left(Q, D_{m}, V\right)$. A $c$-instance $\mathcal{T}$ is a minimal $c$-instance viably complete (resp. strongly complete) for $Q$ relative to $\left(D_{m}, V\right)$ if there exists $\mathcal{I} \in \operatorname{Mod}(\mathcal{T})(\operatorname{resp}$. for all $\mathcal{I} \in \operatorname{Mod}(\mathcal{T}))$ such that $\mathcal{I}$ is a minimal instance complete for a query $Q$.

To define minimal instances in the weak model, we write $(T, \xi) \subsetneq\left(T^{\prime}, \xi^{\prime}\right)$ if $T \subsetneq T^{\prime}$ and $\xi$ is the restriction of $\xi^{\prime}$ on $T$, that is, if for each valuation $\mu^{\prime}$ of $\left(T^{\prime}, \xi^{\prime}\right), \mu(T) \subsetneq \mu^{\prime}\left(T^{\prime}\right)$, and if $\mu^{\prime}\left(T^{\prime}\right)$ satisfies $\xi^{\prime}$, then $\mu(\mathcal{T})$ must satisfy $\xi$, where $\mu$ is the restriction of $\mu^{\prime}$ on $T$. For $\mathcal{T}=\left(T_{1}, \ldots, T_{n}\right)$ and $\mathcal{T}^{\prime}=\left(T_{1}^{\prime}, \ldots, T_{n}^{\prime}\right)$, we write $\mathcal{T} \subsetneq \mathcal{T}^{\prime}$ if $T_{i} \subseteq T_{i}^{\prime}$ for all $i \in[1, n]$, and $T_{j} \subsetneq T_{j}^{\prime}$ for some $j \in[1, n]$.

A database $\mathcal{T}$ is a minimal instance weakly complete for $Q$ relative to $\left(D_{m}, V\right)$ if $\mathcal{T}$ is in $\operatorname{RCQ}\left(Q, D_{m}, V\right)$ and there exists no $\mathcal{T}^{\prime} \subsetneq \mathcal{T}$ such that $\mathcal{T}^{\prime}$ is in $\operatorname{RCQ}\left(Q, D_{m}, V\right)$. Note that $\mathcal{T}^{\prime}$ can be either a $c$-instance or a ground instance.

Example 2.4. Recall $D_{m}, V$ and $Q_{2}$ from Example 2.2. As argued there, a ground instance $D$ is minimally strongly complete for $Q_{2}$ when $D$ consists of a single tuple $t$ with $t$ [NHS] $=$ '915-15-321.' Hence, minimal complete instances may not be unique. In contrast, $D$ is a minimal instance weakly complete for $Q_{2}$ if $D$ is empty. As shown in Example 2.3, the $c$-instance $T$ of Figure 1 is strongly complete for $Q_{1}$. However, it is not minimal: removing $t_{2}-t_{5}$ from $T$ yields a smaller complete database. 


\subsection{Deciding Relative Completeness}

We study three problems associated with relatively complete databases, parameterized with a query language $\mathcal{L}_{Q}$.

\begin{tabular}{|ll|}
\hline $\operatorname{RCDP}\left(\mathcal{L}_{Q}\right):$ & The relatively complete database problem. \\
INPUT: & A query $Q$ in $\mathcal{L}_{Q}$, master data $D_{m}$, a set $V$ of CCs, and a partially closed \\
& $c$-instance $\mathcal{T}$ with regard to $\left(D_{m}, V\right)$. \\
QUESTION: & Is $\mathcal{T}$ in $\operatorname{RCQ}\left(Q, D_{m}, V\right) ?$
\end{tabular}

That is, does $\mathcal{T}$ have complete information to answer $Q$ ?
$\operatorname{RCQP}\left(\mathcal{L}_{Q}\right)$ :
The relatively complete query problem.
INPUT:
$Q, D_{m}$, and $V$ as in RCDP.
QUESTION:
Is $\operatorname{RCQ}\left(Q, D_{m}, V\right)$ nonempty?

It is to determine whether there exists a $c$-instance with complete information to answer $Q$.

\begin{tabular}{|ll|}
\hline $\operatorname{MINP}\left(\mathcal{L}_{Q}\right):$ & The minimality problem. \\
INPUT: & $Q, D_{m}, V$, and $\mathcal{T}$ as in RCDP. \\
QUESTION: & Is $\mathcal{T}$ a minimal $c$-instance complete for $Q$ relative to $\left(D_{m}, V\right) ?$ \\
\hline
\end{tabular}

This asks whether $\mathcal{T}$ is a minimal-size database complete for $Q$, that is, removing any tuple from $\mathcal{T}$ makes it incomplete.

We study these problems when $\mathcal{L}_{Q}$ ranges over the following query languages (e.g., see Abiteboul et al. [1995], for the details):

- $\mathrm{CQ}$, the class of conjunctive queries built up from atomic formulas, that is, relation atoms in the schema $\mathcal{R}$, equality $(=)$ and inequality $(\neq)$, by closing under conjunction $\wedge$ and existential quantification $\exists$;

-UCQ, union of conjunctive queries of the form $Q_{1} \cup \cdots \cup Q_{k}$, where, for each $i \in[1, k]$, $Q_{i}$ is in $\mathrm{CQ}$;

$-\exists \mathrm{FO}^{+}$, first-order logic (FO) queries built from atomic formulas, by closing under $\wedge$, disjunction $\vee$ and $\exists$;

—FO queries built from atomic formulas using $\wedge, \vee$, negation $\neg, \exists$, and universal quantification $\forall$; and

-FP, an extension of $\exists \mathrm{FO}^{+}$with an inflational fix-point operator, that is, queries defined as a collection of rules $p(\vec{x}) \leftarrow p_{1}\left(\vec{x}_{1}\right), \ldots, p_{m}\left(\vec{x}_{m}\right)$, where each $p_{i}$ is either an atomic formula or an IDB predicate.

We also investigate the special case for ground instances. In this setting, $\operatorname{RCQP}\left(\mathcal{L}_{Q}\right)$ is to decide, given $Q$ in $\mathcal{L}_{Q}, D_{m}$, and $V$, whether there exists a ground instance in $\operatorname{RCQ}\left(Q, D_{m}, V\right)$. Similarly, $\operatorname{RCDP}\left(\mathcal{L}_{Q}\right)$ and $\operatorname{MINP}\left(\mathcal{L}_{Q}\right)$ can be stated for ground instances.

We study these problems when $\operatorname{RCQ}\left(Q, D_{m}, V\right)$ is the set of instances that are strongly, weakly, or viably complete, in Sections 4,5 , and 6 , respectively.

The notations used in this article are summarized in Table II.

\section{ANALYSIS OF PARTIALLY CLOSED DATABASES}

Before we study the decision problems for relative completeness, we investigate some basic problems in connection with integrity constraints and partially closed databases. 
Table II. Notations

\begin{tabular}{|c|c|}
\hline Symbols & Notations \\
\hline$(T, \xi)$ & $c$-table, where $T$ is a tableau and $\xi(t)$ is a condition for $\forall t \in T$ \\
\hline$\mu(T)$ & valuation: $\{\mu(t) \mid t \in T$ and $\xi(\mu(t))$ evaluates to true $\}$ \\
\hline $\mathcal{T}$ & c-instance $\left(T_{1}, \ldots, T_{n}\right)$ of schema $\mathcal{R}$ \\
\hline$\left(\mathcal{I}, D_{m}\right) \models \phi$ & a ground instance $\mathcal{I}$ and master data $D_{m}$ satisfy a $\operatorname{CC} \phi$ \\
\hline $\operatorname{Ext}\left(\mathcal{I}, D_{m}, V\right)$ & partially closed extensions of $\mathcal{I}:\left\{\mathcal{I}^{\prime} \mid \mathcal{I} \subsetneq \mathcal{I}^{\prime},\left(\mathcal{I}^{\prime}, D_{m}\right)=V\right\}$ \\
\hline $\operatorname{Mod}\left(\mathcal{T}, D_{m}, V\right)$ & $\left\{\mu(\mathcal{T}) \mid \mu\right.$ is a valuation and $\left.\left(\mu(\mathcal{T}), D_{m}\right) \models V\right\}$ \\
\hline $\mathrm{RCQ}^{s}\left(Q, D_{m}, V\right)$ & $\begin{array}{l}\text { the set of all strongly complete } c \text {-instances } \mathcal{T} \text { of } \mathcal{R} \text { for } Q \text { with regard to }\left(D_{m}, V\right) \text { : } \\
\qquad\left\{\mathcal{T} \mid \forall \mathcal{I} \in \operatorname{Mod}(\mathcal{T}), \forall \mathcal{I}^{\prime} \in \operatorname{Ext}(\mathcal{I})\left(Q(\mathcal{I})=Q\left(\mathcal{I}^{\prime}\right)\right)\right\}\end{array}$ \\
\hline $\mathrm{RCQ}^{w}\left(Q, D_{m}, V\right)$ & $\begin{array}{l}\text { the set of all weakly complete } c \text {-instances } \mathcal{T} \text { of } \mathcal{R} \text { for } Q \text { with regard to }\left(D_{m}, V\right) \text { : } \\
\qquad \bigcap_{\mathcal{I} \in \operatorname{Mod}(\mathcal{T})} Q(\mathcal{I})=\bigcap_{\mathcal{I} \in \operatorname{Mod}(\mathcal{I}), \mathcal{I}^{\prime} \in \operatorname{Ext}(\mathcal{I})} Q\left(\mathcal{I}^{\prime}\right)\end{array}$ \\
\hline $\operatorname{RCQ}^{v}\left(Q, D_{m}, V\right)$ & $\begin{array}{l}\text { the set of all viably complete } c \text {-instances } \mathcal{T} \text { of } \mathcal{R} \text { for } Q \text { with regard to }\left(D_{m}, V\right) \text { : } \\
\qquad \exists \mathcal{I} \in \operatorname{Mod}(\mathcal{T}) \text { such that for } \forall \mathcal{I}^{\prime} \in \operatorname{Ext}(\mathcal{I}), Q(\mathcal{I})=Q\left(\mathcal{I}^{\prime}\right) .\end{array}$ \\
\hline $\begin{array}{c}\operatorname{RCDP}^{s}\left(\mathcal{L}_{Q}\right) \\
\left(\operatorname{RCDP}^{w}\left(\mathcal{L}_{Q}\right) \text { or } \operatorname{RCDP}^{v}\left(\mathcal{L}_{Q}\right)\right)\end{array}$ & $\begin{array}{l}\text { the relatively complete database problem in strong (weak or viable) } \\
\text { completeness model }\end{array}$ \\
\hline $\begin{array}{c}\operatorname{RCQP}^{s}\left(\mathcal{L}_{Q}\right) \\
\left(\operatorname{RCQP}^{w}\left(\mathcal{L}_{Q}\right) \text { or } \operatorname{RCQP}^{v}\left(\mathcal{L}_{Q}\right)\right) \\
\end{array}$ & $\begin{array}{l}\text { the relatively complete query problem in strong (weak or viable) } \\
\text { completeness model }\end{array}$ \\
\hline $\begin{array}{c}\operatorname{MINP}^{s}\left(\mathcal{L}_{Q}\right) \\
\left(\operatorname{MINP}^{w}\left(\mathcal{L}_{Q}\right) \text { or } \operatorname{MINP}^{v}\left(\mathcal{L}_{Q}\right)\right) \\
\end{array}$ & the minimality problem in strong (weak or viable) completeness model \\
\hline $\mathcal{L}_{Q}$ & $\mathrm{CQ}, \mathrm{UCQ}, \exists \mathrm{FO}^{+}, \mathrm{FO}$ or $\mathrm{FP}$ \\
\hline
\end{tabular}

The impact of integrity constraints. Several classes of constraints have been used to specify data consistency, notably, denial constraints and conditional functional dependencies (CFDs) (see Chomicki [2007] and Fan [2008] for surveys). As shown in Fan and Geerts [2009, 2010b], denial constraints and CFDs can be expressed as CCs defined in Section 2 when $\mathcal{L}_{Q}$ is $\mathrm{CQ}$. Hence, we can enforce both relative information completeness and data consistency using those CCs.

One might want to adopt a class $\mathcal{C}$ of constraints that is more powerful than $\mathrm{CCs}$ defined in CQ. However, such $\mathcal{C}$ has an immediate impact on the analysis of relative completeness. For example, it is shown in Fan and Geerts [2009, 2010b] that inclusion dependencies (INDs) can be expressed as CCs in FO. We show later that, when $\mathcal{C}$ consists of, for example, FDs and $\operatorname{INDs}$, both $\operatorname{RCDP}\left(\mathcal{L}_{Q}\right)$ and $\operatorname{RCQP}\left(\mathcal{L}_{Q}\right)$ are undecidable for any language $\mathcal{L}_{Q}$, even in the absence of missing values.

We first introduce a couple of notions. In the presence of a set $\Theta$ of constraints in $\mathcal{C}$, by a partially closed database $\mathcal{I}$, we mean a database that is partially closed in the usual sense, and $\mathcal{I}$ satisfies $\Theta$. Similarly, partially closed extensions of $\mathcal{I}$ are also required to satisfy the additional constraints in $\Theta$. More specifically, consider master data $D_{m}$, a set $V$ of $\mathrm{CCs}$, a set $\Theta$ of constraints in $\mathcal{C}$, and a database schema $\mathcal{R}$.

-A ground instance $\mathcal{I}$ of $\mathcal{R}$ is said to be partially closed relative to $\left(D_{m}, V, \Theta\right)$ if $\left(D_{m}, \mathcal{I}\right) \models$ $V$ and $\mathcal{I} \models \Theta$. That is, $\mathcal{I}$ is partially bounded by $D_{m}$ via $V$ and $\mathcal{I}$ is consistent with regard to the CCs in $V$ and the additional constraints in $\Theta$.

-A ground instance $\mathcal{I}^{\prime}$ of $\mathcal{R}$ is said to be a partially closed extension of $\mathcal{I}$ relative to $\left(D_{m}, V, \Theta\right)$ if $\mathcal{I} \subsetneq \mathcal{I}^{\prime},\left(D_{m}, \mathcal{I}^{\prime}\right)=V$, and $\mathcal{I}^{\prime} \models \Theta$.

-A ground instance $\mathcal{I}$ of $\mathcal{R}$ is said to be complete for a query $Q$ relative to $\left(D_{m}, V, \Theta\right)$ if it is partially closed and for each partially closed extension $\mathcal{I}^{\prime}$ of $\mathcal{I}, Q(\mathcal{I})=Q\left(\mathcal{I}^{\prime}\right)$. We use $\operatorname{RCQ}\left(Q, D_{m}, V, \Theta\right)$ to denote the set of ground instances that are complete for a query $Q$ relative to $\left(D_{m}, V, \Theta\right)$. 
PROPOSITION 3.1. In the presence of both FDs and INDs, for ground instances, RCDP and RCQP are undecidable even when $\mathcal{L}_{Q}$ is $C Q$, and master data $D_{m}$ and the set $V$ of CCs are both empty.

Proof. To prove Proposition 3.1, it suffices to show that RCDP(CQ) and RCQP(CQ) are undecidable, since $\mathrm{CQ}$ is contained in $\mathcal{L}_{Q}$, when $\mathcal{L}_{Q}$ is $\mathrm{UCQ}, \exists \mathrm{FO}^{+}, \mathrm{FO}$, or FP.

We verify the undecidability of $R C D P(C Q)$ and $R C Q P(C Q)$ by reduction from the implication problem for FDs and INDs. In particular, we consider instances $(\Theta, \varphi)$ of the implication problem, where $\Theta$ is a set of FDs and INDs defined on a database schema $\mathcal{R}$, and $\varphi$ is an FD $X \rightarrow A$ defined on a relation schema $R$ in $\mathcal{R}$. It is undecidable to determine, given such $(\Theta, \varphi)$, whether $\Theta \models \varphi$, that is, whether, for every instance $\mathcal{I}_{\mathcal{R}}$ of $\mathcal{R}$, if $\mathcal{I}_{\mathcal{R}} \models \Theta$, then $\mathcal{I}_{\mathcal{R}} \models \varphi$ (see Abiteboul et al. [1995]).

(1) RCDP $(\mathrm{CQ})$. Given an instance $(\Theta, \varphi)$ of the implication problem, we define a Boolean query $Q$ in $\mathrm{CQ}$ as follows:

$$
Q()=\exists \vec{x}, \vec{y}_{1}, \vec{y}_{2}, w, w^{\prime}\left(R\left(\vec{x}, w, \vec{y}_{1}\right) \wedge R\left(\vec{x}, w^{\prime}, \vec{y}_{2}\right) \wedge w \neq w^{\prime}\right),
$$

where $\vec{x}$ corresponds to attributes $X$ in $R, w$ and $w^{\prime}$ both correspond to attribute $A$ in $R$, as specified by the $\mathrm{FD} \varphi:(X \rightarrow A)$ on $R$, and $\vec{y}_{1}$ and $\vec{y}_{2}$ encode attributes $R \backslash(X \cup\{A\})$. Intuitively, for an instance $\mathcal{I}_{\mathcal{R}}$ of $\mathcal{R}$, the query $Q$ returns true if $I_{R} \not \forall \varphi$, that is, when there exist tuples $t_{1}, t_{2}$ in $I_{R}$ such that $t_{1}[X]=t_{2}[X]$ but $t_{1}[A] \neq t_{2}[A]$; otherwise, $Q$ returns false. Moreover, we set $D_{m}$ and $V$ both to be empty.

Consider an instance $\mathcal{I}_{\emptyset}$ of $\mathcal{R}$ consisting of empty relations only. We show that $\mathcal{I}_{\emptyset}$ is in $\operatorname{RCQ}\left(Q, D_{m}, \Theta, V\right)$ if and only if $\Theta \models \varphi$. First, assume that $\Theta \models \varphi$. Then, for all instances of $\mathcal{I}_{\mathcal{R}}$ of $\mathcal{R}$, if $\mathcal{I}_{\mathcal{R}}=\Theta$, then $\mathcal{I}_{\mathcal{R}} \models \varphi$, hence, $Q$ returns false. Therefore, $\mathcal{I}_{\emptyset}$ is complete for $Q$ relative to $\left(D_{m}, V, \Theta\right)$. Conversely, assume that $\Theta \not \varphi$. Then, there exists an instance $\mathcal{I}_{\mathcal{R}}$ of $\mathcal{R}$ such that $\mathcal{I}_{\mathcal{R}} \models \Theta$ but $\mathcal{I}_{\mathcal{R}} \forall \models \varphi$. Obviously, $\mathcal{I}_{R}$ is not empty. Then, $Q$ returns true, which differs from $Q\left(\mathcal{I}_{\emptyset}\right)$. In addition, $\mathcal{I}_{\mathcal{R}}$ is a partially closed extension of $\mathcal{I}_{\emptyset}$ since $V$ is empty. From these, it follows that $\mathcal{I}_{\emptyset}$ is not in $\operatorname{RCQ}\left(Q, D_{m}, V, \Theta\right)$.

(2) RCQP $(\mathrm{CQ})$. Given an instance $(\Theta, \varphi)$ of the implication problem, we define a $\mathrm{CQ}$ query $Q$ and a set $\Theta^{\prime}$ of INDs and FDs, such that $\Theta \models \varphi$ if and only if $\operatorname{RCQ}\left(Q, D_{m}, V, \Theta^{\prime}\right)$ is nonempty, where master data $D_{m}$ and the set $V$ of CCs are empty.

To define $\Theta^{\prime}$ and $Q$, we use a database schema $\mathcal{R}^{\prime}$ that extends $\mathcal{R}$ by adding a new attribute $G$ to every relation schema in $\mathcal{R}$, where $\operatorname{dom}(G)$ is infinite. The schema $\mathcal{R}^{\prime}$ also includes the unary relation $E$ that consists of a single attribute of an infinite domain. The set $\Theta^{\prime}$ consists of FDs and INDs constructed as follows.

-For each FD $Y \rightarrow B$ in $\Theta$, the FD $([G, Y] \rightarrow B)$ is in $\Theta^{\prime}$.

-For each IND $R_{1}\left[Y_{1}\right] \subseteq R_{2}\left[Y_{2}\right]$ in $\Theta$, the IND $R_{1}\left[G, Y_{1}\right] \subseteq R_{2}\left[G, Y_{2}\right]$ is in $\Theta^{\prime}$.

Similarly, we rewrite the FD $\varphi: X \rightarrow A$ as $\varphi^{\prime}:([G, X] \rightarrow A)$. Intuitively, for each instance $\mathcal{I}_{\mathcal{R}^{\prime}}$ of $\mathcal{R}^{\prime}$, if we group tuples of $\mathcal{I}_{\mathcal{R}^{\prime}}$ by the attribute $G$, then $\mathcal{I}_{\mathcal{R}^{\prime}}$ is partitioned into a collection of groups $\mathcal{I}_{g}$, where $g$ ranges over elements in $\operatorname{dom}(G)$ that appear in the $G$-attribute of $\mathcal{I}_{\mathcal{R}^{\prime}}$. One can readily verify that $\mathcal{I}_{\mathcal{R}^{\prime}} \models \Theta^{\prime}$ if and only if for each group $\mathcal{I}_{g}, \mathcal{I}_{g} \models \Theta$. Similarly, $\mathcal{I}_{\mathcal{R}^{\prime}} \models \varphi^{\prime}$ if and only if $\mathcal{I}_{g} \models \varphi$ for each group $\mathcal{I}_{g}$.

The CQ query $Q$ is similar to its counterpart given these facts. It is defined as follows:

$$
Q(z)=E(z) \wedge \exists g, \vec{x}, \vec{y}_{1}, \vec{y}_{2}, w, w^{\prime}\left(R\left(g, \vec{x}, w, \vec{y}_{1}\right) \wedge R\left(g, \vec{x}, w^{\prime}, \vec{y}_{2}\right) \wedge w \neq w^{\prime}\right) .
$$

This query detects whether there exist tuples $t_{1}$ and $t_{2}$ violating the FD $\varphi^{\prime}$. That is, it checks whether there exist $t_{1}$ and $t_{2}$ from the same group (with the same value in their $G$ attributes) such that $t_{1}$ and $t_{2}$ violate $\varphi$. If so, then $Q$ returns the instance $I_{E}$ of $E$.

We next show that $\Theta \models \varphi$ if and only if $\operatorname{RCQ}\left(Q, D_{m}, V, \Theta^{\prime}\right)$ is nonempty. Assume first that $\Theta \models \varphi$. Then, one can readily verify that, for every instance $\mathcal{I}_{\mathcal{R}^{\prime}}$ of $\mathcal{R}^{\prime}$, if $\mathcal{I}_{\mathcal{R}^{\prime}}=\Theta^{\prime}$, 
then $Q\left(\mathcal{I}_{\mathcal{R}^{\prime}}\right)$ is empty. As a result, every instance $\mathcal{I}_{\mathcal{R}^{\prime}}$ is in $\operatorname{RCQ}\left(Q, D_{m}, V, \Theta^{\prime}\right)$. Conversely, assume that $\Theta \forall \forall$. Then, there exists an instance $\mathcal{I}_{\mathcal{R}}$ such that $\mathcal{I}_{\mathcal{R}}=\Theta$ but $\mathcal{I}_{\mathcal{R}} \not \models \varphi$. Assume, by contradiction, that there exists $\mathcal{I}_{\mathcal{R}^{\prime}} \in \operatorname{RCQ}\left(Q, D_{m}, V, \Theta^{\prime}\right)$. We construct a partially closed extension $\mathcal{I}_{\mathcal{R}^{\prime}}^{\prime}$, as follows. Let $g$ be a distinct value that does not appear in any $G$ column of $\mathcal{I}_{\mathcal{R}^{\prime}}$. Define $\mathcal{I}_{\mathcal{R}^{\prime}}^{\prime}$ such that, for each relation $S$ in $\mathcal{R}$, its instance in $\mathcal{I}_{\mathcal{R}^{\prime}}^{\prime}$ is the union of $I^{\prime} \cup\left(\left\{t_{g}\right\} \times I\right)$, where $I^{\prime}, I$ are the instances of $S$ in $\mathcal{I}_{\mathcal{R}^{\prime}}$ and $\mathcal{I}_{\mathcal{R}}$, respectively, and $t_{g}$ is a unary tuple with a single attribute $G$ such that $t[G]=g$. In addition, the instance $I_{E}^{\prime}$ of schema $E$ in $\mathcal{I}_{\mathcal{R}^{\prime}}^{\prime}$ properly contains its counterpart $I_{E}$ in $\mathcal{I}_{\mathcal{R}^{\prime}}$. Obviously $\mathcal{I}_{\mathcal{R}^{\prime}}^{\prime} \models \Theta^{\prime}$, that is, $\mathcal{I}_{\mathcal{R}^{\prime}}^{\prime}$ is indeed a partially closed extension of $\mathcal{I}_{\mathcal{R}^{\prime}}$. However, $Q\left(\mathcal{I}_{\mathcal{R}^{\prime}}^{\prime}\right)$ is $I_{E}^{\prime}$, which is by no means equal to the answer to $Q$ in $\mathcal{I}_{\mathcal{R}^{\prime}}$, since the latter is either $\emptyset$ or $I_{E}$. This contradicts the assumption that $\operatorname{RCQ}\left(Q, D_{m}, V, \Theta^{\prime}\right)$ is nonempty.

Note that, in these proofs, both master data and the set $V$ of CCs are empty, that is, they are independent of the instance $(\Theta, \varphi)$ of the implication problem considered.

The undecidability result suggests that we consider integrity constraints that are expressible as CCs in $\mathrm{CQ}$, to focus on the complexity incurred by the analysis of relative completeness rather than by integrity constraints. As remarked earlier, CCs are powerful enough to express constraints often used in data cleaning.

Reasoning about $\boldsymbol{c}$-instances. As remarked earlier, the analysis of relative completeness requires decision procedures for determining some basic problems in connection with partially closed $c$-instances, which are stated as follows.

- The consistency problem is to determine, given master data $D_{m}$, a set $V$ of CCs and a $c$-instance $\mathcal{T}$, whether $\operatorname{Mod}\left(\mathcal{T}, D_{m}, V\right)$ is nonempty.

- The extensibility problem is to determine, given master data $D_{m}$, a set $V$ of CCs and a ground instance $\mathcal{I}$, whether $\operatorname{Ext}\left(\mathcal{I}, D_{m}, V\right)$ is nonempty, that is, whether $\mathcal{I}$ can be extended without violating $V$.

In the sequel, we assume that queries are defined over a single relation. This does not lose generality due to the following lemma. For a database schema $\mathcal{R}$, we denote by inst $(\mathcal{R})$ the set of all ground instances of $\mathcal{R}$.

Lemma 3.2. For every database schema $\mathcal{R}=\left(R_{1}, \ldots, R_{n}\right)$, there exist a single relation schema $R$, a linear-time computable bijective function $f_{D}$ from $\operatorname{inst}(\mathcal{R})$ to inst $(R)$, a lineartime computable function $f_{Q}: \mathcal{L}_{Q} \rightarrow \mathcal{L}_{Q}$, and a linear-time computable function $f_{\mathcal{C}}$ from CCs to CCs, such that

(a) for all instances $\mathcal{I}$ of $\mathcal{R}$ and any query $Q \in \mathcal{L}_{Q}$ over $\mathcal{R}, Q(\mathcal{I})=f_{Q}(Q)\left(f_{D}(\mathcal{I})\right)$; and

(b) for every set $V$ of CCs and master data $D_{m},\left(\mathcal{I}, D_{m}\right) \models V$ if and only if $\left(f_{D}(\mathcal{I}), D_{m}\right) \models$ $f_{\mathcal{C}}(V)$, where $f_{\mathcal{C}}(V)=\left\{f_{\mathcal{C}}(\psi) \mid \psi \in V\right\}$.

Here, $\mathcal{L}_{Q}$ ranges over $C Q, U C Q, \exists F O^{+}, F O$, and $F P$.

Proof. We assume, without loss of generality, that all relations $R_{i}$ in $\mathcal{R}$ correspond to the same schema $R^{\prime}$. Indeed, one can make the relations $R_{i}$ uniform by renaming attributes and adding dummy attributes. Consider a distinct attribute $A_{R}$ that takes values from $\operatorname{dom}(A)=[1, n]$. Define $R$ to be an extension of $R^{\prime}$ by adding attribute $\left(A_{R}: \operatorname{dom}(A)\right)$. We define $f_{D}, f_{Q}$, and $f_{\mathcal{C}}$ as follows.

(1) Define $f_{D}$ such that, for every instance $\mathcal{I}=\left(I_{1}, \ldots, I_{n}\right)$ of $\mathcal{R}, f_{D}(\mathcal{I})=\bigcup_{j \in[1, n]} I_{j} \times$ $\left\{\left(A_{R}=j\right)\right\}$. The function $f_{D}$ is clearly bijective.

(2) For a query language $\mathcal{L}_{Q}$, define $f_{Q}$ such that, given a query $Q \in \mathcal{L}_{Q}$ defined on $\mathcal{R}, f_{Q}(Q)$ substitutes $R\left(A_{R}=i, \vec{x}\right)$ for every occurrence of $R_{i}(\vec{x})$ in $Q$, that is, it replaces every occurrence of $R_{i}$ with a Project-Select expression $\pi_{\operatorname{att}\left(R_{i}\right)}\left(\sigma_{A_{R}=i}(R)\right)$, where $\operatorname{attr}\left(R_{i}\right)$ denotes the set of attributes in $R_{i}$. 


$$
I_{(01)}=\left|\begin{array}{c}
X \\
1 \\
0
\end{array}\right| \quad I_{\vee}=\left|\begin{array}{ccc}
A_{1} & A_{2} & B \\
0 & 0 & 0 \\
0 & 1 & 1 \\
1 & 0 & 1 \\
1 & 1 & 1
\end{array}\right| \quad I_{\wedge}=\left|\begin{array}{ccc}
A_{1} & A_{2} & B \\
0 & 0 & 0 \\
0 & 1 & 0 \\
1 & 0 & 0 \\
1 & 1 & 1
\end{array}\right| \quad I_{\neg}=\left|\begin{array}{cc}
A & \bar{A} \\
0 & 1 \\
1 & 0
\end{array}\right|
$$

Fig. 2. Ground relations used in the lower-bound proofs of Proposition 3.3.

(3) Similarly, we define $f_{\mathcal{C}}$ such that, for every CC $\psi, f_{\mathcal{C}}(\psi)$ substitutes $R\left(A_{R}=i, \vec{x}\right)$ for every occurrence of $R_{i}(\vec{x})$ in $\psi$.

It is then readily verified that (a) $Q(\mathcal{I})=f_{Q}(Q)\left(f_{D}(\mathcal{I})\right)$ and (b) for all master data $D_{m},\left(\mathcal{I}, D_{m}\right) \models \psi$ if and only if $\left(f_{D}(\mathcal{I}), D_{m}\right) \models f_{\mathcal{C}}(\psi)$, and then $\left(\mathcal{I}, D_{m}\right) \models V$ if and only if $\left(f_{D}(\mathcal{I}), D_{m}\right)=f_{\mathcal{C}}(V)$. Furthermore, $f_{D}, f_{Q}$, and $f_{\mathcal{C}}$ can be computed in linear time.

Proposition 3.3. The consistency and extensibility problems are both $\Sigma_{2}^{p}$-complete. The complexity is unchanged even in the absence of local conditions in c-instances and when master data $D_{m}$ is fixed.

Proof. We show that the consistency problem and the extensibility problem are both $\Sigma_{2}^{p}$-complete.

(1) The consistency problem. We show that, given master data $D_{m}$, a set $V$ of CCs and a $c$-instance $\mathcal{T}$, it is $\Sigma_{2}^{p}$-complete to decide whether $\operatorname{Mod}\left(\mathcal{T}, D_{m}, V\right)$ is nonempty.

Lower bound. We show that the complement of the consistency problem, that is, the problem to decide whether $\operatorname{Mod}\left(\mathcal{T}, D_{m}, V\right)$ is empty, is $\Pi_{2}^{p}$-hard. From this, it follows that the consistency problem is $\Sigma_{2}^{p}$-hard. We verify the $\Pi_{2}^{p}$-hardness by reduction from the $\forall^{*} \exists^{*} 3$ SAT problem, which is known to be $\Pi_{2}^{p}$-complete (see Papadimitriou [1994]). The $\forall^{*} \exists^{*}$ 3sAT problem is to determine, given a sentence $\varphi=\forall X \exists Y \psi(X, Y)$, whether $\varphi$ is true. Here $X=\left\{x_{1}, \ldots, x_{n}\right\}, Y=\left\{y_{1}, \ldots, y_{m}\right\}$, and $\psi$ is an instance of 3sat, that is, $\psi=C_{1} \wedge \cdots \wedge C_{r}$ and, for each $i \in[1, r]$, clause $C_{i}$ is of the form $\ell_{1}^{i} \vee \ell_{2}^{i} \vee \ell_{3}^{i}$, where for each $l \in[1,3], \ell_{l}^{i}$ is either a variable or the negation of a variable in $X \cup Y$.

Given $\varphi=\forall X \exists Y \psi(X, Y)$, we define a database schema $\mathcal{R}$, a $c$-instance $\mathcal{T}$ of $\mathcal{R}$, master data $D_{m}$, and a set $V$ of CCs, such that $\varphi$ is true if and only if $\operatorname{Mod}\left(\mathcal{T}, D_{m}, V\right)$ is empty. We construct $\mathcal{R}, \mathcal{T}, D_{m}$ and $V$ as follows.

(a) The database schema $\mathcal{R}$ consists of five relation schemas: $R_{(0,1)}(X), R_{\vee}\left(A_{1}, A_{2}, B\right)$, $R_{\wedge}\left(A_{1}, A_{2}, B\right), \quad R_{\neg}(A, \bar{A}), \quad$ and $\quad R_{X}\left(X_{1}, \ldots, X_{n}\right)$. Intuitively, $\quad R_{(0,1)}(X), \quad R_{\vee}\left(A_{1}, A_{2}, B\right)$, $R_{\wedge}\left(A_{1}, A_{2}, B\right)$, and $R_{\neg}(A, \bar{A})$ are to store constant relations encoding truth values, disjunction, conjunction, and negation of variables, respectively. We use $R_{X}\left(X_{1}, \ldots, X_{n}\right)$ to generate a truth assignment for variables in $X$.

(b) We construct a $c$-instance $\mathcal{T}=\left(I_{(0,1)}, I_{\vee}, I_{\wedge}, I_{\neg}, T_{X}\right)$ in which $I_{(0,1)}, I_{\vee}, I_{\wedge}$, and $I_{\neg}$ are ground relations, as shown in Figure 2, to encode the Boolean domain, disjunction, conjunction, and negation, respectively, such that $\psi$ can be expressed in CQ in terms of these relations, while $T_{X}=\left(\left\{\left(x_{1}, \ldots, x_{n}\right)\right\}\right.$, true $)$ is a $c$-table defined in terms of variables in $X$, without any local conditions.

(c) Master data $D_{m}$ is specified by five relation schemas: $R_{(0,1)}^{m}(X), R_{\vee}^{m}\left(A_{1}, A_{2}, B\right)$, $R_{\wedge}^{m}\left(A_{1}, A_{2}, B\right), R_{\neg}^{m}(A, \bar{A})$, and $R_{\emptyset}^{m}(W)$. Intuitively, $R_{(0,1)}^{m}, R_{\vee}^{m}, R_{\wedge}^{m}$, and $R_{\neg}^{m}$ are the same as $R_{(0,1)}(X), R_{\vee}\left(A_{1}, A_{2}, B\right), R_{\wedge}\left(A_{1}, A_{2}, B\right)$, and $R_{\neg}(A, \bar{A})$ to store constant relations encoding Boolean values, disjunction, conjunction, and negation of variables, respectively 
(denoted by $R_{(0,1)}^{m}=R_{(0,1)}, R_{\vee}^{m}=R_{\vee}, R_{\wedge}^{m}=R_{\wedge}, R_{\neg}^{m}=R_{\neg}$, respectively). The master data instances consist of $I_{(0,1)}^{m}=I_{(0,1)}, I_{\vee}^{m}=I_{\vee}, I_{\wedge}^{m}=I_{\wedge}, I_{\neg}^{m}=I_{\neg}$, and $I_{\emptyset}^{m}=\emptyset$.

(d) The set $V$ consists of the following CCs:

$-R_{(0,1)} \subseteq R_{(0,1)}^{m}, R_{\vee} \subseteq R_{\vee}^{m}, R_{\wedge} \subseteq R_{\wedge}^{m}, R_{\neg} \subseteq R_{\neg}^{m}$; that is, the tables in $\mathcal{T}$ encoding the Boolean values and operations are fixed;

-for each $i \in[1, n], \exists x_{1} \ldots x_{i-1} x_{i+1} \ldots x_{n} R_{X}\left(x_{1}, \ldots, x_{n}\right) \subseteq R_{(0,1)}^{m}$; these ensure that each instance of $R_{X}$ encodes a valid truth assignment for $X$;

$-q(w) \subseteq R_{\emptyset}^{m}(w)$, with $q(w)=\exists \vec{x}, \vec{y}\left(Q_{X}(\vec{x}) \wedge Q_{Y}(\vec{y}) \wedge Q_{\psi}(\vec{x}, \vec{y}, w) \wedge w=1\right)$. Here, $Q_{X}(\vec{x})=$ $R_{X}\left(x_{1}, \ldots, x_{n}\right)$ picks a truth assignment for $X$, and $Q_{Y}(\vec{y})$ is $R_{(0,1)}\left(y_{1}\right) \wedge \cdots \wedge R_{(0,1)}\left(y_{m}\right)$ in CQ, that is, it constructs all possible truth assignments of variables in $Y$ by means of $m-1$ Cartesian Products of $I_{(0,1)}$. Furthermore, given a truth assignment $\left(\mu_{X}, \mu_{Y}\right)$ of $(X, Y)$, the subquery $Q_{\psi}\left(\mu_{X}, \mu_{Y}, w\right)$ is to evaluate $\psi\left(\mu_{X}, \mu_{Y}\right)$ by recording its truth value in $w$, which is either 0 or 1 . While CQ supports neither disjunction nor negation, $Q_{\psi}$ can encode $\psi$ in CQ by leveraging relations $I_{\neg}, I_{\vee}$, and $I_{\wedge}$. The query $q(w)$ returns $\{(1)\}$ if and only if $\psi\left(\mu_{X}, \mu_{Y}\right)$ evaluates true, where $\mu_{X}$ and $\mu_{Y}$ are the truth assignment selected by $Q_{X}(\vec{x})$ and $Q_{Y}(\vec{y})$, respectively.

Intuitively, for each ground instance $\mathcal{I}=\left(I_{(0,1)}, I_{\vee}, I_{\wedge}, I_{\neg}, I_{X}\right)$ of $\mathcal{T},\left(\mathcal{I}, D_{m}\right) \models V$ if and only if, under the truth assignment $\mu_{X}$ of $X$ variables encoded by $I_{X}$, there exists no truth assignment $\mu_{Y}$ of $Y$ variables that makes $\psi$ true.

We next show that $\varphi$ is false if and only if $\operatorname{Mod}\left(\mathcal{T}, D_{m}, V\right)$ is not empty.

$\Rightarrow$ First, assume that $\varphi$ is false. Then, there exists a truth assignment $\mu_{X}^{0}$ of $X$ such that there exists no truth assignment $\mu_{Y}$ that makes $\psi\left(\mu_{X}^{0}, \mu_{Y}\right)$ true. Let $\mathcal{I}_{\mu}=\mu(\mathcal{T})=$ $\left(I_{(0,1)}, I_{\vee}, I_{\wedge}, I_{\neg}, \mu\left(T_{X}\right)\right)$ such that $\mu\left(T_{X}\right)$ agrees with $\mu_{X}^{0}$. As discussed earlier, $\mathcal{I}_{\mu}$ is in $\operatorname{Mod}\left(\mathcal{T}, D_{m}, V\right)$. Hence, $\operatorname{Mod}\left(\mathcal{T}, D_{m}, V\right)$ is nonempty.

$\Leftarrow$ Conversely, suppose that $\varphi$ is true. Then, for all truth assignments $\mu_{X}$ of $X$, there exists a truth assignment $\mu_{Y}$ such that $\psi\left(\mu_{X}, \mu_{Y}\right)$ evaluates to true. Hence, for all ground instances $\mathcal{I}_{\mu}=\left(I_{(0,1)}, I_{\vee}, I_{\wedge}, I_{\neg}, \mu\left(T_{X}\right)\right)$, where $\mu$ sets $x_{i}$ to 0 or 1 (thus all possible truth assignments of $X$ are considered), $q\left(\mathcal{I}_{\mu}\right)$ returns $\{(1)\}$. This violates the $\mathrm{CC} q(w) \subseteq$ $R_{\emptyset}^{m}(w)$. Hence, $\operatorname{Mod}\left(\mathcal{T}, D_{m}, V\right)$ is empty.

Upper bound. We next provide a $\Sigma_{2}^{p}$ algorithm that, given master data $D_{m}$, a set $V$ of CCs and a $c$-instance $\mathcal{T}$ as input, returns "yes" if $\operatorname{Mod}\left(\mathcal{T}, D_{m}, V\right)$ is nonempty.

By Lemma 3.2, we assume without loss of generality that $\mathcal{R}$ consists of a single relation schema and $\mathcal{T}$ is a $c$-table $(T, \xi)$. To develop the algorithm, we need the following notations.

-We define Adom to be $S \cup$ New $\cup \mathbf{d}_{f}$, where (a) $S$ consists of all constants that appear in $T, D_{m}$, or $V$; (b) New is a set of fresh values that are not in $S$, one for each variable in $T$ or $V$; and (c) $\mathbf{d}_{f}$ is the set including all the values in the finite domains of attributes $A$ in the relation schema that have a finite domain. Intuitively, Adom consists of all constants in the active domains of $\mathcal{T}, D_{m}$, or $V$ and all constants appearing in the domains of attributes with finite domain. We will show that, for consistency checking, it suffices to consider valuations of a $c$-table drawing values from Adom only.

-A valuation $\mu$ of a c-table $(T, \xi)$ on Adom is a valuation of $(T, \xi)$ in which, for each variable $x$ in $T, \mu(x)$ is in Adom and $\mu$ is the identity mapping on constants in $T$. Furthermore, if $x$ appears in an attribute of finite domain, $\mu(x)$ takes values in this finite domain. Note that finite domain values are included in Adom. We use $\mu(T)$ to denote the ground instance $\{\mu(t) \mid t \in T, \xi(\mu(t))$ evaluates to true $\}$. 
-We use $\operatorname{Mod}_{\text {Adom }}\left(T, D_{m}, V\right)$ to denote the set $\{\mu(T) \mid \mu$ is a valuation on Adom, and $\left.\left(\mu(T), D_{m}\right) \models V\right\}$. We also write $\operatorname{Mod}_{\text {Adom }}\left(T, D_{m}, V\right)$ as $\operatorname{Mod}_{\text {Adom }}(T)$ if $D_{m}$ and $V$ are clear from the context.

Using these notations, we give the algorithm as follows. It checks whether there exists a valuation $\mu$ of $(T, \xi)$ such that $\left(\mu(T), D_{m}\right) \models V$.

(1) Guess a valuation $\mu$ of $(T, \xi)$ on Adom.

(2) Check whether $\left(\mu(T), D_{m}\right) \models V$. If so, return "yes"; otherwise, reject the guess.

The algorithm is in $\Sigma_{2}^{p}$ since it involves guessing a valuation (in NP) combined with a call to a coNP oracle in Step 2. Step 2 is in coNP since the CCs in $V$ are defined with CQ queries; hence, checking whether $\left(\mu(T), D_{m}\right) \not \models V$ can be done in NP as follows.

(1) Guess a constraint $q(\mathcal{R}) \subseteq p\left(\mathcal{R}_{m}\right)$ in $V$ and let $\left(T_{q}, u_{q}\right)$ be the tableau representing $q$; guess a valuation $\mu_{q}$ of variables in $T_{q}$ that takes values from $\mu(T)$.

(2) Check whether $\mu_{q}\left(u_{q}\right) \notin p\left(D_{m}\right)$; if so, return "no"; otherwise, reject the guess.

Obviously, the algorithm returns "no" if and only if there exists a constraint that is not satisfied by $\mu(T)$ and $D_{m}$. Moreover, the algorithm is in NP since its second step is in PTIME. Hence, the consistency problem is in $\Sigma_{2}^{p}=\mathrm{NP}^{\mathrm{NP}}=\mathrm{NP}^{\mathrm{coNP}}$.

The correctness of the algorithm for consistency checking is ensured by the following property: $\operatorname{Mod}\left(T, D_{m}, V\right)$ is nonempty if and only if $\operatorname{Mod}_{\text {Adom }}\left(T, D_{m}, V\right)$ is nonempty. Hence, the algorithm returns "yes" only when $\operatorname{Mod}\left(T, D_{m}, V\right)$ is nonempty.

We next verify the property. If $\operatorname{Mod}_{\text {Adom }}\left(T, D_{m}, V\right)$ is nonempty, then there exists a valuation $\mu$ of $(T, \xi)$ on Adom such that $\left(\mu(T), D_{m}\right) \models V$. Then, obviously $\mu(T)$ is in $\operatorname{Mod}\left(T, D_{m}, V\right)$; hence, $\operatorname{Mod}\left(T, D_{m}, V\right)$ is nonempty. Conversely, suppose that there exists an instance $I$ in $\operatorname{Mod}\left(T, D_{m}, V\right)$. Then, there exists a valuation $v$ of $(T, \xi)$ such that $I=v(T)$. We next turn $v$ into a valuation $\mu$ of $(T, \xi)$ on Adom, showing that $\operatorname{Mod}_{\text {Adom }}\left(T, D_{m}, V\right)$ is nonempty. More precisely, we define $\mu$ such that, for every variable $x$ in $T, \mu(x)=v(x)$ if $v(x) \in$ Adom; otherwise, $\mu(x)$ takes a value in New, such that it preserves the equality on variables, that is, for all other variables $y$ in $T, \mu(x)=\mu(y)$ if and only if $v(x)=v(y)$. This is possible since, by the definition of New, this set contains new constants for every variable in $T$. We need to verify that $\left(\mu(T), D_{m}\right) \models V$. Let $q(\mu(T)) \subseteq p\left(D_{m}\right)$ be any $\mathrm{CC}$ in $V$, and $\left(T_{q}, u_{q}\right)$ be the tableau representing $q$. Then, for each valuation $\mu_{q}^{\prime}$ of variables in $T_{q}$ that draws values from $\mu(\mathcal{T})$, by the definition of $\mu(T)$, there exists a valuation $\mu_{q}$ of the variables such that $\mu_{q}$ agrees with $\mu_{q}^{\prime}$ on variables that are not assigned a New-value, but take values from $v(T)$ outside of Adom, for the remaining variables. Recall that $\nu(\mathcal{T}) \in \operatorname{Mod}\left(T, D_{m}, V\right)$. Thus, $\mu_{q}\left(u_{q}\right)$ is in $p\left(D_{m}\right)$. One can readily verify that $\mu_{q}^{\prime}\left(u_{q}\right)$ is in $p\left(D_{m}\right)$. As a result, $\left(\mu(T), D_{m}\right) \models V$; hence, $\mu(T)$ is in $\operatorname{Mod}_{\text {Adom }}\left(T, D_{m}, V\right)$.

(2) The extensibility problem. We next show that, given $D_{m}, V$, and a ground instance $\overline{\mathcal{I}}$, it is $\Sigma_{2}^{p}$-complete to decide whether $\operatorname{Ext}\left(\mathcal{I}, D_{m}, V\right)$ is nonempty.

Lower bound. We show that it is $\Pi_{2}^{p}$-hard to decide whether $\operatorname{Ext}\left(\mathcal{I}, D_{m}, V\right)$ is empty. This is again verified by reduction from the $\forall^{*} \exists^{*}$ 3st problem. Given an instance $\varphi=$ $\forall X \exists Y \psi(X, Y)$ of $\forall^{*} \exists^{*} 3$ sat, we use the same $\mathcal{R}, D_{m}$, and $V$ given in (1), and let $\mathcal{I}_{0}=$ $\left(I_{(0,1)}, I_{\vee}, I_{\wedge}, I_{\neg}, I_{X}^{\emptyset}\right)$, where $I_{X}^{\emptyset}$ is empty.

We next show that $\varphi$ is true if and only if $\operatorname{Ext}\left(\mathcal{I}_{0}, D_{m}, V\right)$ is empty.

$\Rightarrow$ First, assume that $\varphi$ is true. Then, for all truth assignments $\mu_{X}$ of $X$, there exists a truth assignment $\mu_{Y}$ of $Y$ such that $\psi\left(\mu_{X}, \mu_{Y}\right)$ evaluates to true. Hence, for all extensions $\mathcal{I}_{0}^{\prime}=\left(I_{(0,1)}^{\prime}, I_{\vee}^{\prime}, I_{\wedge}^{\prime}, I_{\neg}^{\prime}, I_{X}^{\prime}\right)$ of $\mathcal{I}_{0}$, as long as $I_{(0,1)}^{\prime}=I_{(0,1)}, I_{\vee}^{\prime}=I_{\vee}, I_{\wedge}^{\prime}=I_{\wedge}$, 
$I_{\neg}^{\prime}=I_{\neg}$, and $I_{X}^{\prime}$ encodes a truth assignment of $X, q\left(I_{X}^{\prime}\right)$ returns $\{(1)\}$. This, however, violates the $\operatorname{CC} q(w) \subseteq R_{\emptyset}^{m}(w)$; hence, $\operatorname{Ext}\left(\mathcal{I}_{0}, D_{m}, V\right)$ is empty.

$\Leftarrow$ Conversely, assume that $\varphi$ is false. Let $\mathcal{I}_{0}^{\prime}=\left(I_{(0,1)}, I_{\vee}, I_{\wedge}, I_{\neg}, I_{X}^{0}\right)$, where $I_{X}^{0}$ is an instance of $R_{X}$ consisting of a single truth assignment $\mu_{X}^{0}$ of $X$ such that $\exists Y \psi\left(\mu_{X}^{0}, Y\right)$ is false. Then, along the same lines as the previous proof, one can easily verify that $\mathcal{I}_{0}^{\prime}$ is in $\operatorname{Ext}\left(\mathcal{I}_{0}, D_{m}, V\right)$.

Upper bound. We now develop a $\Sigma_{2}^{p}$ algorithm that takes master data $D_{m}$, a set $V$ of CCs, and a ground instance $I$ as input, and returns "yes" if $\operatorname{Ext}\left(I, D_{m}, V\right)$ is not empty.

To present the algorithm, we assume without loss of generality that $I$ is an instance of a relation schema $R$, by Lemma 3.2. Recall the definition of Adom given in the upper bound proof for the consistency problem, except that $\mathcal{T}$ is replaced with the ground instance $I$. The algorithm works as follows.

(1) Guess a single tuple $t$ of $R$ with values from Adom that does not belong to $I$.

(2) Check whether $\left(I \cup\{t\}, D_{m}\right) \models V$. If so, return "yes"; otherwise, reject the guess.

Following the same argument as the upper bound proof for the consistency problem, one can verify that the algorithm is in $\Sigma_{2}^{p}$. To show that the algorithm is correct, observe the following. (a) There exists an extension $I^{\prime}$ in $\operatorname{Ext}\left(I, D_{m}, V\right)$ if and only if there exists a single tuple $t$ such that $I \cup\{t\}$ is in $\operatorname{Ext}\left(I, D_{m}, V\right)$. This can be easily verified based on the monotonicity of CQ queries that define the CCs in $V$. (b) There exists a tuple $t$ such that $I \cup\{t\}$ is in $\operatorname{Ext}\left(I, D_{m}, V\right)$ if and only if there exists a tuple $t^{\prime}$ with values in Adom such that $I \cup\left\{t^{\prime}\right\}$ is in $\operatorname{Ext}\left(I, D_{m}, V\right)$. This can be verified along the same lines as the upper bound proof for the consistency problem given what has been presented. Putting these together, we conclude that the algorithm returns "yes" if $\operatorname{Ext}\left(I, D_{m}, V\right)$ is nonempty.

\section{STRONG RELATIVE INFORMATION COMPLETENESS}

We next study RCDP, RCQP, and MINP for strongly relatively complete databases, that is, databases in which neither missing values nor missing tuples prevent them from having complete information for answering queries relative to master data. We refer to these problems as $\operatorname{RCDP}^{s}, \mathrm{RCQP}^{s}$, and $\mathrm{MINP}^{s}$, respectively. Recall that $\operatorname{RCQ}^{s}\left(Q, D_{m}, V\right)$ denotes the set of instances that are strongly complete for $Q$ with regard to $\left(D_{m}, V\right)$.

We establish complexity bounds on these problems for $c$-instances. For ground instances, we give complexity results for $\operatorname{MINP}^{s}\left(\mathcal{L}_{Q}\right)$ not considered in Fan and Geerts [2010b], and for the cases of $\operatorname{RCQP}^{s}\left(\mathcal{L}_{Q}\right)$ that were left open in Fan and Geerts [2010b].

Our main conclusion about the strong completeness model is that missing values make our lives harder, but not too much.

\subsection{The Relatively Complete Database Problem in the Strong Model}

This problem is to decide whether a given database is relatively complete for a query. It is known that, for ground instances, $\operatorname{RCDP}^{s}\left(\mathcal{L}_{Q}\right)$ is undecidable when $\mathcal{L}_{Q}$ is $\mathrm{FO}$ or $\mathrm{FP}$, and it is $\Pi_{2}^{p}$-complete when $\mathcal{L}_{Q}$ ranges over $\mathrm{CQ}, \mathrm{UCQ}$, and $\exists \mathrm{FO}^{+}[\mathrm{Fan}$ and Geerts 2009]. The following result tells us that the presence of missing values does not complicate the analysis: all the results for ground instances remain the same for $c$-instances.

In practice, master data $D_{m}$ and the set $V$ of CCs are often predefined and fixed, and only databases and user queries vary. One might think that $\mathrm{RCDP}^{s}$ would become simpler in this setting. This is not the case, however: the complexity bounds remain intact when $D_{m}$ and $V$ are fixed. 
Theorem 4.1. For c-instances, $\operatorname{RCDP}^{s}\left(\mathcal{L}_{Q}\right)$ is

-undecidable when $\mathcal{L}_{Q}$ is either $F O$ or FP, and

$-\Pi_{2}^{p}$-complete when $\mathcal{L}_{Q}$ is $C Q, U C Q$, or $\exists F O^{+}$.

The complexity bounds remain unchanged when master data $D_{m}$ and the set $V$ of CCs are fixed.

Proof. We first show that $\operatorname{RCDP}^{s}\left(\mathcal{L}_{Q}\right)$ is undecidable when $\mathcal{L}_{Q}$ is FO or FP. We then show that the problem becomes $\Pi_{2}^{p}$-complete when $\mathcal{L}_{Q}$ is $\mathrm{CQ}$, $\mathrm{UCQ}$, or $\exists \mathrm{FO}^{+}$.

(1) When $\mathcal{L}_{Q}$ is FO or FP. The $\operatorname{RCDP}^{s}(\mathrm{FO})$ and $\mathrm{RCDP}^{s}(\mathrm{FP})$ for ground instances have been proved to be undecidable with fixed $D_{m}$ and CCs by reduction from the satisfiability problem of FO and the emptiness problem for 2-head DFA, respectively (Theorem 3.1 [Fan and Geerts 2010b]; see the details of these two problems in the proofs of Theorem 5.1(2) and Lemma 4.6, respectively). This undecidability carries over to $\operatorname{RCDP}^{s}(\mathrm{FO})$ and $\operatorname{RCDP}^{s}(\mathrm{FP})$ for $c$-instances since ground instances are also $c$-instances.

(2) When $\mathcal{L}_{Q}$ is $\mathrm{CQ}, \mathrm{UCQ}$, or $\exists \mathrm{FO}^{+}$. We next show that $\operatorname{RCDP}^{s}\left(\mathcal{L}_{Q}\right)$ is $\Pi_{2}^{p}$-complete when $\mathcal{L}_{Q}$ is $\mathrm{CQ}$, UCQ, or $\exists \mathrm{FO}^{+}$.

Lower bound. It is known that $\mathrm{RCDP}^{s}(\mathrm{CQ})$ for ground instances is $\Pi_{2}^{p}$-hard with fixed master data and CCs by reduction from the $\forall^{*} \exists^{*} 3 \mathrm{SAT}$ problem (Theorem 3.6 [Fan and Geerts 2010b]; see the details of the latter problem in Proposition 3.3(1)). The lower bound thus carries over since ground instances are $c$-instances.

Upper bound. We next show that $\operatorname{RCDP}^{s}(\mathrm{CQ}), \mathrm{RCDP}^{s}(\mathrm{UCQ})$, and $\mathrm{RCDP}^{s}\left(\exists \mathrm{FO}^{+}\right)$are all in $\Pi_{2}^{p}$ for $c$-instances. We first provide a $\Pi_{2}^{p}$ algorithm for testing strongly complete $c$-instances for CQ queries. Later, we show how the algorithm can be extended to UCQ and $\exists \mathrm{FO}^{+}$. We first present the algorithm for $\operatorname{RCDP}^{s}(\mathrm{CQ})$.

$\operatorname{RCDP}^{s}(C Q)$. To show that $\operatorname{RCDP}^{s}(C Q)$ is in $\Pi_{2}^{p}$, we first provide a characterization of $c$ instances that are strongly complete for CQ queries. Based on the characterization, we then provide a $\Pi_{2}^{p}$ algorithm for testing strongly complete $c$-instances for CQ queries.

Consider a CQ query $Q$, master data $D_{m}$, a set $V$ of CCs and a $c$-instance $\mathcal{T}=(T, \xi)$. By Lemma 3.2, we assume, without loss of generality, that $Q$ is defined over a relation schema $R$, and $\mathcal{T}=(T, \xi)$ is a $c$-table of $R$.

The characterization is defined in terms of the following notations.

- The CQ query $Q$ can be expressed as a tableau query $\left(T_{Q}, u_{Q}\right)$, where $T_{Q}$ denotes atomic formulas in $Q$ and $u_{Q}$ is the output summary (e.g., see Abiteboul et al. [1995] for details). Observe that $T_{Q}$ can be regarded as a $c$-table without local conditions.

- Similarly, we define a set of constants as in the proof of Proposition 3.3(1), also referred to as Adom, including constants in $Q, \mathcal{T}, D_{m}$ or $V$, new distinct values in New that are not in $Q, \mathcal{T}, D_{m}$, and $V$, one for each variable in $Q, \mathcal{T}$, or $V$, and a set of constants $\mathbf{d}_{f}$ corresponding to constants in the domains of finite domain attributes. It differs from its counterpart in the proof of Proposition 3.3 in that it includes constants in query $Q$.

-Furthermore, a ground instance $I$ of $R$ is said to be bounded by $\left(D_{m}, V\right)$ if, for each $I^{\prime} \in \operatorname{Mod}_{\text {Adom }}\left(I \cup T_{Q}, D_{m}, V\right), Q(I)=Q\left(I^{\prime}\right)$ [Fan and Geerts 2010b].

The following lemma characterizes the strongly complete $c$-instances for CQ queries.

LEMMA 4.2. For every query $Q$ in $C Q$, master data $D_{m}$, set $V$ of $C C s$, and c-instance $(T, \xi),(T, \xi)$ is in $\operatorname{RCQ}^{s}\left(Q, D_{m}, V\right)$ if and only if, for each $I \in \operatorname{Mod}_{\text {Adom }}\left(T, D_{m}, V\right), I$ is bounded by $\left(D_{m}, V\right)$. 
Proof. It suffices to show the following. (1) For each ground instance $I$ of $R, I$ is in $\operatorname{RCQ}^{s}\left(Q, D_{m}, V\right)$ if and only if it is bounded by $\left(D_{m}, V\right)$. (2) The $c$-instance $(T, \xi)$ is in $\operatorname{RCQ}^{s}\left(Q, D_{m}, V\right)$ if and only if, for each $I \in \operatorname{Mod}_{\text {Adom }}\left(T, D_{m}, V\right), I$ is in $\operatorname{RCQ}^{s}\left(Q, D_{m}, V\right)$. (1) We first show that $I$ is in $\operatorname{RCQ}^{s}\left(Q, D_{m}, V\right)$ if and only if, for each $I^{\prime} \in \operatorname{Mod}_{\text {Adom }}\left(I \cup T_{Q}\right)$, $Q(I)=Q\left(I^{\prime}\right)$. First, suppose that $I$ is complete for $Q$. Then, for every $I^{\prime} \in \operatorname{Ext}(I)$, $Q(I)=Q\left(I^{\prime}\right)$. In particular, for every $I^{\prime} \in \operatorname{Mod}_{\text {Adom }}\left(I \cup T_{Q}\right), Q(I)=Q\left(I^{\prime}\right)$ since $I^{\prime}$ is also in $\operatorname{Ext}(I)$. Conversely, suppose that $I$ is not complete for $Q$. Then, there must exist an $I^{\prime} \in \operatorname{Ext}(I)$ such that $Q(I) \neq Q\left(I^{\prime}\right)$. More specifically, since $Q$ is monotonic, there must exist a valuation $v$ of $T_{Q}$ that draws values from $I^{\prime}$, such that $Q\left(v\left(T_{Q}\right)\right) \nsubseteq Q(I)$. Define a valuation $v^{\prime}$ such that, for every variable $x$ in $T_{Q}, v^{\prime}(x)$ is a distinct value in New if $v(x)$ is not in Adom, and $v^{\prime}(x)=v(x)$ otherwise. Observe the following. (a) The valuation $v^{\prime}$ draws values from Adom. (b) $Q\left(v^{\prime}\left(T_{Q}\right)\right) \nsubseteq Q(I)$, by the choice of the values in New and the assumption that $Q\left(v\left(T_{Q}\right)\right) \nsubseteq Q Q(I)$. (c) $\left(I \cup v^{\prime}\left(T_{Q}\right), D_{m}\right) \models V$. This follows from the assumption that $I^{\prime} \in \operatorname{Ext}(I), I \cup v\left(T_{Q}\right) \subseteq I^{\prime}$, and $\left(I^{\prime}, D_{m}\right) \models V$. We then also have that $\left(I \cup v\left(T_{Q}\right), D_{m}\right) \models V$ since the CCs in $V$ are defined in terms of monotonic CQ queries. Then, again by the choice of the values in New, $\left(I \cup v^{\prime}\left(T_{Q}\right), D_{m}\right) \models V$. Putting (a), (b), and (c) together, we have that $I \cup v^{\prime}\left(T_{Q}\right)$ is in $\operatorname{Mod}_{\text {Adom }}\left(I \cup T_{Q}\right)$ but $Q(I) \neq Q\left(I \cup v^{\prime}\left(T_{Q}\right)\right)$.

(2) We next show that $(T, \xi)$ is in $\operatorname{RCQ}^{s}\left(Q, D_{m}, V\right)$ if and only if, for each $I \in \operatorname{Mod}_{\text {Adom }}(T$, $\left.D_{m}, V\right), I$ is in $\operatorname{Mod}\left(T, D_{m}, V\right)$. First, assume that $(T, \xi)$ is in $\operatorname{RCQ}^{s}\left(Q, D_{m}, V\right)$. Then, by the definition of strongly complete $c$-instances, all ground instances in $\operatorname{Mod}\left(T, D_{m}, V\right)$ are complete for $Q$ relative to $\left(D_{m}, V\right)$, including those in $I \in \operatorname{Mod}_{\text {Adom }}\left(T, D_{m}, V\right)$. Conversely, assume that $(T, \xi)$ is not in $\operatorname{RCQ}^{s}\left(Q, D_{m}, V\right)$. Then, there exists a valuation $\mu$ of $T$ such that $I_{1}=\mu(T), I_{1} \in \operatorname{Mod}\left(T, D_{m}, V\right)$, and there exists $I_{2} \in \operatorname{Ext}\left(I_{1}\right)$ such that $Q\left(I_{1}\right) \subsetneq Q\left(I_{2}\right)$. Then, along the same lines as the argument given for (1), one can verify that there exist valuations $\mu^{\prime}$ and $v^{\prime}$ that draw values from Adom such that $I_{1}^{\prime}=\mu^{\prime}(T)$, $I_{1}^{\prime} \in \operatorname{Mod}\left(T, D_{m}, V\right), I_{2}^{\prime}=I_{1}^{\prime} \cup v^{\prime}\left(T_{Q}\right), I_{2}^{\prime} \in \operatorname{Ext}\left(I_{1}^{\prime}\right)$, but $Q\left(I_{1}^{\prime}\right) \subsetneq Q\left(I_{2}^{\prime}\right)$. The valuations $\mu^{\prime}$ and $\nu^{\prime}$ are constructed by leveraging the choice of the values in New. That is, there exists an $I \in \operatorname{Mod}_{\text {Adom }}\left(T, D_{m}, V\right)$ such that $I$ is not complete for $Q$ relative to $\left(D_{m}, V\right)$.

With this characterization in place, we now present a $\Sigma_{2}^{p}$ algorithm for the complement of our problem: given $(T, \xi), D_{m}, V$, and CQ query $Q$, it returns "yes" if there exists a database $I \in \operatorname{Mod}_{\text {Adom }}\left(T, D_{m}, V\right)$ and a tuple $s$ such that $s \notin Q(I)$ but $s \in Q\left(I^{\prime}\right)$ for some $I^{\prime} \in \operatorname{Mod}_{\text {Adom }}\left(I \cup T_{Q}\right)$; otherwise, it returns "no." More specifically, the algorithm does the following:

(1) Guess a valuation $\mu$ of $(T, \xi)$ on Adom, a valuation $v$ for $T_{Q}$ taking values from Adom, and a tuple $s$ of $R_{Q}$, where $R_{Q}$ is the schema of the query result $Q(D)$.

(2) Check:

(a) whether $\mu(T) \in \operatorname{Mod}\left(T, D_{m}, V\right)$; if so, continue; otherwise, reject the current guess; this test can be done in coNP;

(b) whether $\left(\mu(T) \cup v\left(T_{Q}\right)\right) \in \operatorname{Ext}\left(\mu(T), D_{m}, V\right)$; if so, continue; otherwise, reject the current guess; this test can be done in coNP;

(c) whether $s \notin Q(\mu(T))$; if so, continue; otherwise, reject the current guess; this test can be done in coNP;

(d) whether $s \in Q\left(\mu(T) \cup v\left(T_{Q}\right)\right)$; if so, return "yes"; otherwise, reject the current guess; this test can be done in NP

The complexity of the algorithm is thus in $\Sigma_{2}^{p}$; hence, $\operatorname{RCDP}^{s}(\mathrm{CQ})$ is in $\Pi_{2}^{p}$. We now verify the correctness of the algorithm. Clearly, the algorithm returns "yes" if a counterexample to the strong completeness of $(T, \xi)$ for $Q$ is found. The counterexample consists of $I=\mu(T)$ and $I^{\prime}=I \cup v\left(T_{Q}\right)$, where $\mu$ and $v$ are the guesses that lead to a successful run of the algorithm. Conversely, we show that if $(T, \xi)$ is incomplete for $Q$ relative to $\left(D_{m}, V\right)$, then the algorithm returns “yes." By Lemma 4.2 , if $(T, \xi)$ is 
incomplete, then there exist a valuation $\mu$ of $T$ with Adom and a valuation $v$ of $T_{Q}$ with Adom, such that $I=\mu(T), I^{\prime}=I \cup v\left(T_{Q}\right), I \in \operatorname{Mod}_{\text {Adom }}\left(T, D_{m}, V\right)$, and $I^{\prime} \in \operatorname{Mod}_{\text {Adom }}(I \cup$ $\left.v\left(T_{Q}\right), D_{m}, V\right)$, but there exists a tuple $s \in Q\left(I^{\prime}\right)$ and $s \notin Q(I)$. Such valuations $\mu$ and $v$ can be guessed by the algorithm. That is, the algorithm is able to find a counterexample.

$\operatorname{RCDP}(U C Q)$. We next show that it is in $\Pi_{2}^{p}$ to decide whether a $c$-instance is strongly complete for queries in UCQ. Consider a query $Q$ in UCQ: $Q_{1} \cup \cdots \cup Q_{k}$, where $Q_{i}$ is a query in $\mathrm{CQ}$ for each $i \in[1, k]$. Consider master data $D_{m}$, a set $V$ of CCs, and a $c$-instance $(T, \xi)$. We represent $Q_{i}$ as a tableau query $\left(T_{i}, u_{i}\right)$ for $i \in[1, k]$. We revise the notion of bounded databases for UCQ queries as follows: a ground instance $I$ of $R$ is said to be bounded by $\left(D_{m}, V\right)$ if, for each $i \in[1, k]$ and each $I^{\prime} \in \operatorname{Mod}_{\text {Adom }}\left(I \cup T_{i}\right)$, $Q(I)=Q\left(I^{\prime}\right)$.

Along the same lines as Lemma 4.2, we have the following characterization for strongly complete $c$-instances for UCQ queries.

Lemma 4.3. For every query $Q$ in UCQ, master data $D_{m}$, any set $V$ of $C C s$, and any cinstance $(T, \xi),(T, \xi)$ is in $\mathrm{RCQ}^{S}\left(Q, D_{m}, V\right)$ if and only if, for each $I \in \operatorname{Mod}_{\mathrm{Adom}}\left(T, D_{m}, V\right)$, $I$ is bounded by $\left(D_{m}, V\right)$.

Proof. It suffices to show that, for a UCQ query $Q=Q_{1} \cup \cdots \cup Q_{k}$, (1) for each ground instance $I$ of $R, I$ is complete for $Q$ relative to $\left(D_{m}, V\right)$ if and only if it is bounded by $\left(D_{m}, V\right)$, that is, for each $i \in[1, k]$ and each $I^{\prime} \in \operatorname{Mod}_{\text {Adom }}\left(I \cup T_{i}\right), Q(I)=$ $Q\left(I^{\prime}\right)$, where $\left(T_{i}, u_{i}\right)$ is the tableau representation of $Q_{i}$; and (2) the $c$-instance $(T, \xi)$ is in $\operatorname{RCQ}^{s}\left(Q, D_{m}, V\right)$ if and only if, for each $I \in \operatorname{Mod}_{\text {Adom }}\left(T, D_{m}, V\right), I$ is complete for $Q$ relative to $\left(D_{m}, V\right)$. These can be verified along the same lines as the proof of Lemma 4.2.

With this characterization, we extend the $\Sigma_{2}^{p}$ algorithm given earlier to UCQ queries. More specifically, the algorithm presented earlier needs only a minor modification: In Step 2, we additionally guess one of the component queries $Q_{i}$ in $Q$ and a valuation $v_{i}$ of $Q_{i}$ 's tableau $T_{i}$; furthermore, Steps $2(\mathrm{~b})$ and $2(\mathrm{~d})$ use $v_{i}\left(T_{i}\right)$ rather than $v(T)$. In other words, the algorithm tries to find a $Q_{i}$ and instances $I \in \operatorname{Mod}_{\text {Adom }}\left(T, D_{m}, V\right)$ and $I^{\prime} \in \operatorname{Mod}_{\text {Adom }}\left(I \cup v_{i}\left(T_{i}\right), D_{m}, V\right)$ for which $Q(I) \subsetneq Q\left(I^{\prime}\right)$. That is, the algorithm verifies whether there is an $I \in \operatorname{Mod}_{\text {Adom }}\left(T, D_{m}, V\right)$ that is not bounded by $\left(D_{m}, V\right)$.

This modification does not affect the complexity of the algorithm; thus, it remains in $\Sigma_{2}^{p}$. Steps 2(c) and $2(\mathrm{~d})$ remain in coNP and NP, respectively, for UCQ queries. The correctness of the algorithm can be verified along the same lines as its counterpart for $\operatorname{RCDP}^{s}(\mathrm{CQ})$, based on Lemma 4.2. This shows that it is in $\Pi_{2}^{p}$ time to determine whether a $c$-instance is strongly complete for a query in UCQ with regard to $\left(D_{m}, V\right)$.

$\operatorname{RCDP}\left(\exists F \mathrm{O}^{+}\right)$. We show that the $\Pi_{2}^{p}$ algorithm given earlier can be extended to $\exists \mathrm{FO}^{+}$queries. A query $Q$ in $\exists \mathrm{FO}^{+}$is equivalent to a possibly exponentially long union of CQ queries. Therefore, an unfolding of the query will bring us beyond $\Pi_{2}^{p}$. However, we can avoid unfolding $Q$ by replacing the guess in Step 2 by obtaining a single CQ by guessing disjunctions in $Q$. As before, this modification does not affect the complexity, as Steps 2(c) and (d) remain in coNP and NP, respectively, for $\exists \mathrm{FO}^{+}$queries. Putting these together, we have a $\Pi_{2}^{p}$ algorithm for checking $\operatorname{RCDP}^{s}\left(\exists \mathrm{FO}^{+}\right)$.

This completes the proof of Theorem 4.1.

Theorem 4.1 is verified for $c$-instances $\mathcal{T}$ for which $\operatorname{Mod}(\mathcal{T})$ is nonempty. We next show that the complexity bounds remain unchanged without assuming that $\operatorname{Mod}(\mathcal{T})$ is nonempty. This obviously holds when $\mathcal{L}_{Q}$ is FO or FP, for which RCDP ${ }^{s}$ is undecidable. For $\mathrm{CQ}, \mathrm{UCQ}$, and $\exists \mathrm{FO}^{+}$, we need an extra step to check whether $\operatorname{Mod}(\mathcal{T})$ is empty; if so, the algorithm terminates with "yes"; otherwise, it checks RCDP" in $\Pi_{2}^{p}$. By 
Proposition 3.3, the initial step is also in $\Pi_{2}^{p}$. Hence, $\mathrm{RCDP}^{s}$ remains in $\Pi_{2}^{p}$ for $\mathrm{CQ}$, $\mathrm{UCQ}$, and $\exists \mathrm{FO}^{+}$. In other words, the assumption has no impact on the complexity of $\mathrm{RCDP}^{s}$.

\subsection{The Relatively Complete Query Problem in the Strong Model}

This problem is to determine whether a given query has a relatively complete database at all. For $\operatorname{RCQP}^{s}\left(\mathcal{L}_{Q}\right)$, we do not have to worry about missing values. $\operatorname{RCQP}^{s}\left(\mathcal{L}_{Q}\right)$ for $c$-instances and its counterpart for ground instances coincide.

Lemma 4.4. For every schema $\mathcal{R}$, query $Q$, master data $D_{m}$, set $V$ of $C C s$, and number $K$, there exists a c-instance $\mathcal{T}$ of $\mathcal{R}$ such that $|\mathcal{T}| \leq K$ and $\mathcal{T} \in \mathrm{RCQ}^{s}\left(Q, D_{m}, V\right)$ if and only if there exists a ground instance $\mathcal{I}$ of $\mathcal{R}$ such that $|\mathcal{I}| \leq K$ and $\mathcal{I} \in \operatorname{RCQ}^{s}\left(Q, D_{m}, V\right)$.

Proof. First, assume that there exists a $c$-instance $\mathcal{T} \in \operatorname{RCQ}^{s}\left(Q, D_{m}, V\right)$, with $|\mathcal{T}| \leq K$. Then, by the definition of strongly complete $c$-instances, $\operatorname{Mod}\left(\mathcal{T}, D_{m}, V\right) \neq \emptyset$; moreover, for each instance $\mathcal{I} \in \operatorname{Mod}\left(\mathcal{T}, D_{m}, V\right), \mathcal{I}$ is complete for $Q$ relative to $\left(D_{m}, V\right)$. Furthermore, $|\mathcal{I}| \leq K$ since $\mathcal{I}=\mu(\mathcal{T})$ for a valuation of $\mathcal{T}$. Conversely, assume that there exists an instance $\mathcal{I}$ in $\operatorname{RCQ}^{s}\left(Q, D_{m}, V\right)$ with $|\mathcal{I}| \leq K$. Then, $\mathcal{I}$ is also a $c$-instance itself, which is complete for $Q$ relative to $\left(D_{m}, V\right)$.

Consequently, one needs to consider only $\operatorname{RCQP}{ }^{s}\left(\mathcal{L}_{Q}\right)$ for ground instances. Nevertheless, for ground instances, the complexity bounds on $\operatorname{RCQP}^{s}\left(\mathcal{L}_{Q}\right)$ were left open in Fan and Geerts [2009] when $\mathcal{L}_{Q}$ is FO or FP, and when CCs are expressed in CQ. RCQP $\left(\mathcal{L}_{Q}\right)$ was shown undecidable in Fan and Geerts [2009] by using CCs expressed as fixed FO or FP queries. We settle these cases here.

Theorem 4.5. For c-instances, $\operatorname{RCQP}^{s}\left(\mathcal{L}_{Q}\right)$ is

-undecidable when $\mathcal{L}_{Q}$ is $F O$ or FP; and

-NEXPTIME-complete when $\mathcal{L}_{Q}$ is $C Q, U C Q$, or $\exists F O^{+}$.

The complexity bounds remain unchanged when $D_{m}$ and $V$ are fixed.

Proof. In light of Lemma 4.4, it suffices to consider ground instances of $\mathcal{R}$.

(1) When $\mathcal{L}_{Q}$ is FO. We show that $\operatorname{RCQP}^{s}(\mathrm{FO})$ is undecidable by reduction from the satisfiability problem for FO, which is undecidable (see Trakhtenbrot [1950] and Abiteboul et al. [1995]). Given an FO query $q$, we construct master data $D_{m}$, a set $V$ of CCs, and an FO query $Q$, such that $q$ is satisfiable if and only if $\operatorname{RCQ}^{s}\left(Q, D_{m}, V\right)$ is empty.

We now define $D_{m}, V$, and $Q$. The reduction does not rely on master data, that is, $V$ and $D_{m}$ are empty. To define $Q$, by Lemma 3.2, assume without loss of generality that $q$ is defined over a relation schema $R$. We define another schema $R^{\prime}$, where $R^{\prime}$ extends $R$ by adding an extra attribute $A$ with an infinite domain. We define $Q$ as the following query over $R^{\prime}$ :

$$
Q(I)= \begin{cases}\emptyset & \text { if } \forall a\left(q\left(\pi_{R}\left(\sigma_{A=a}(I)\right)\right)=\emptyset\right) \\ I & \text { otherwise. }\end{cases}
$$

We show that $q$ is satisfiable if and only if $\operatorname{RCQ}^{s}\left(Q, D_{m}, V\right)$ is empty.

$\Rightarrow$ First, suppose that $q$ is satisfiable, and let $I$ be an instance of $R$ such that $q(I) \neq \emptyset$. For each instance $I_{1}$ of $R^{\prime}$, define $I_{2}=I_{1} \cup(\{A=b\} \times I) \cup\{t\}$, where $b$ is a distinct constant not appearing in $I_{1}$, and $t$ is a tuple not in $I_{1}$ and with $t[A] \neq b$. Here, $t$ ensures that $I_{1} \neq I_{2}$ even when $I$ is empty. Then, $I_{2}$ is a partially closed extension of $I_{1}$ but $Q\left(I_{1}\right) \neq Q\left(I_{2}\right) . Q\left(I_{2}\right)=I_{2}$, but $Q\left(I_{1}\right) \neq I_{2}$ no matter whether $Q\left(I_{1}\right)=I_{1}$ or $Q\left(I_{1}\right)=\emptyset$. Thus, $I_{1}$ is not in $\operatorname{RCQ}^{s}\left(Q, D_{m}, V\right)$; hence, $\operatorname{RCQ}^{s}\left(Q, D_{m}, V\right)$ is empty. 
$\Leftarrow$ Conversely, if $q$ is not satisfiable, then $Q(I)=\emptyset$ for every instance $I$ of $R^{\prime}$. Thus, $\operatorname{RCQ}^{s}\left(Q, D_{m}, V\right)$ is not empty, since every instance of $R^{\prime}$ is relatively complete for $Q$.

(2) When $\mathcal{L}_{Q}$ is FP. To show that $\operatorname{RCQP}^{s}(\mathrm{FP})$ is undecidable, we first prove the undecidability of the following problem, from which we will give a reduction to $\operatorname{RCQP}^{s}(\mathrm{FP})$.

The satisfiability problem for FP in the presence of FDs is to determine, given an FP query $p$ defined on schema $\mathcal{R}$ and a set $\Theta$ of FDs defined on $\mathcal{R}$, whether there exists an instance $\mathcal{I}$ of $\mathcal{R}$ such that $\mathcal{I} \models \Theta$ and $p(\mathcal{I})$ is nonempty. The undecidability of this problem was claimed in Levy et al. [1993]. We now provide a proof for a stronger result in that the set $\Theta$ of FDs can be assumed to be fixed.

LEMMA 4.6. The satisfiability problem of FP is undecidable in the presence of a fixed set of FDs.

Proof. We show the undecidability by reduction from the emptiness problem for deterministic finite 2-head automata, which is known to be undecidable Spielmann [2000]. Our proof closely follows the reduction presented in Spielmann [2000, Theorem 3.3.1], which shows that the satisfiability of the existential fragment of transitiveclosure logic, $\mathrm{E}+\mathrm{TC}$, is undecidable over a schema having at least two nonnullary relation schemas, one being a function symbol. Although E+TC allows the negation of atomic expression as opposed to FP, the undecidability proof only uses a very restricted form of negation, which can be simulated using $\neq$ and a fixed set of FDs.

We start with a review of necessary definitions from Spielmann [2000].

A deterministic finite 2-head automaton (2-head DFA) is a quintuple $\mathcal{A}=$ $\left(S, \Sigma, \Delta, s_{0}, s_{\text {acc }}\right)$, consisting of a finite set of states $S$, an input alphabet $\Sigma=\{0,1\}$, an initial state $s_{0}$, an accepting state $s_{\text {acc }}$, and a transition function $\Delta: S \times \Sigma_{\epsilon} \times \Sigma_{\epsilon} \rightarrow$ $S \times\{0,+1\} \times\{0,+1\}$, where $\Sigma_{\epsilon}=\Sigma \cup\{\epsilon\}$.

A configuration of $\mathcal{A}$ is a triple $\left(s, w_{1}, w_{2}\right) \in S \times \Sigma^{*} \times \Sigma^{*}$, representing that $\mathcal{A}$ is in state $s$, and the first and second head of $\mathcal{A}$ are positioned on the first symbol of $w_{1}$ and $w_{2}$, respectively. On an input string $w \in \Sigma^{*}, \mathcal{A}$ starts from the initial configuration $\left(s_{0}, w, w\right)$; the successor configuration is defined as usual.

A 2-head DFA $\mathcal{A}$ accepts $w$ if it can reach a configuration $\left(s_{\text {acc }}, w_{1}, w_{2}\right)$ from the initial configuration for $w$; otherwise, $\mathcal{A}$ rejects $w$. The language accepted by $\mathcal{A}$ is denoted by $L(\mathcal{A})$.

The emptiness problem for 2-head DFAs is to determine, given a 2-head DFA $\mathcal{A}$, whether $L(\mathcal{A})$ is empty.

Given a 2 -head DFA $\mathcal{A}=\left(S, \Sigma, \delta, s_{0}, s_{\text {acc }}\right)$, we define a schema $\mathcal{R}$, an FP-query $\Pi$, and a fixed set $\Theta$ of FDs over $\mathcal{R}$. We show that $L(\mathcal{A})$ is nonempty if and only if there exists an instance $\mathcal{I}$ of $\mathcal{R}$ such that (i) $\mathcal{I} \models \Theta$ and (ii) $\Pi(\mathcal{I})$ is nonempty.

(a) The database schema $\mathcal{R}$ consists of two relations $P(V, A)$ and $S\left(W, A_{1}, A_{2}\right)$. Intuitively, $P(V, A)$ and $S\left(W, A_{1}, A_{2}\right)$ are to store constant relations, encoding a word $w$ in $\Sigma^{*}$. More specifically, an instance $\mathcal{I}=\left(I_{P}, I_{S}\right)$ of $\mathcal{R}$ represents a string $w \in \Sigma^{*}$ such that (i) elements in $\sigma_{V=1}\left(I_{P}\right)$ represent the positions in $w$ where a 1 occurs; (ii) $\sigma_{V=0}\left(I_{P}\right)$ records those positions in $w$ that are 0 ; and (iii) $I_{S}$ encodes a successor relation over these positions in $w$ by $\pi_{A_{1}, A_{2}}\left(\sigma_{A_{1} \neq A_{2}}\left(I_{S}\right)\right) \cup \pi_{A_{1}, A_{2}}\left(\sigma_{A_{1}=A_{2} \wedge W=1}\left(I_{S}\right)\right)$, in which the last part identifies the final position in the successor relation.

We denote $\sigma_{V=1}(P) \cup \sigma_{V=0}(P)$ by FP query $\Pi_{P}$ and $\pi_{A_{1}, A_{2}}\left(\sigma_{A_{1} \neq A_{2}}(S)\right) \cup$ $\pi_{A_{1}, A_{2}}\left(\sigma_{A_{1}=A_{2} \wedge W=1}(S)\right)$ by FP query $\Pi_{S}$.

(b) We will use three FDs to ensure that we only consider those instances of $P$ and $S$ that represent a word in $\Sigma^{*}$, called well-formed instances of $P$ and $S$. An instance $\mathcal{I}=\left(I_{P}, I_{S}\right)$ is well formed if (i) $\sigma_{V=1}\left(I_{P}\right)$ and $\sigma_{V=0}\left(I_{P}\right)$ are disjoint (i.e., a string can have only one letter at each position); and $\pi_{A_{1}, A_{2}}\left(\sigma_{A_{1} \neq A_{2}}\left(I_{S}\right)\right) \cup \pi_{A_{1}, A_{2}}\left(\sigma_{A_{1}=A_{2} \wedge W=1}\left(I_{S}\right)\right)$ must (ii) 
be a function and (iii) contain a unique tuple of the form $(k, k)$ for some constant $k$ indicating the final position.

To ensure this, we require the presence of a tuple $(1, k, k)$ in $I_{S}$. We also require that any instance $I_{S}$ of $S$ contains a tuple of the form $(w, 0, i)$, where 0 represents the initial position and $i$ is some constant. The latter two requirements will be ensured by FP-queries $\Pi_{\text {ini }}$ and $\Pi_{\text {fin }}$, respectively, to be defined shortly.

More specifically, the conditions (i) through (iii) will be enforced by the following set $\Theta$ of FDs:

$-A \rightarrow V$, enforcing that, for every instance $\mathcal{I}^{\prime}=\left(I_{P}^{\prime}, I_{S}^{\prime}\right)$ of $\mathcal{R}$ such that $\mathcal{I}^{\prime} \models \Theta$, condition (i) is satisfied for $I_{P}^{\prime}$.

$-A_{1} \rightarrow A_{2}$, ensuring that $\pi_{A_{1}, A_{2}}\left(I_{S}^{\prime}\right)$ encodes a function; hence, condition (ii) is satisfied.

$-W \rightarrow A_{1}, A_{2}$, ensuring that there can be at most one tuple with its $W$-attribute set to

1 in $I_{S}^{\prime}$. As a result, $\pi_{A_{1}, A_{2}}\left(\sigma_{A_{1}=A_{2} \wedge W=1}\left(I_{S}^{\prime}\right)\right)$ contains at most one tuple, and condition (iii) is satisfied.

In summary, any instance $\mathcal{I}^{\prime}=\left(I_{P}^{\prime}, I_{S}^{\prime}\right)$ of $\mathcal{R}$ that satisfies $\Theta$ is well formed, with the exception that we still need to check for the existence of an initial and a final position in the instance $I_{S}^{\prime}$ of $S$ in $\mathcal{I}^{\prime}$.

(c) Before we define the query $\Pi$, we show, following Spielmann [2000], how the nonemptiness of $L(\mathcal{A})$ can be expressed in terms of an E+TC-formula over $\mathcal{R}$. Consider a transition $\delta \in \Delta$ of the form $\delta=\left(s, \mathrm{in}_{1}, \mathrm{in}_{2}\right) \rightarrow\left(s^{\prime}\right.$, move $_{1}$, move $\left._{2}\right)$. Such a transition can be encoded by means of the conjunctive query

$$
\varphi_{\delta}\left(x, y, z, x^{\prime}, y^{\prime}, z^{\prime}\right)=\left(x=s \wedge x^{\prime}=s^{\prime} \wedge \alpha_{1}(y) \wedge \alpha_{2}(z) \wedge \beta_{1}\left(y, y^{\prime}\right) \wedge \beta_{2}\left(z, z^{\prime}\right)\right) .
$$

Intuitively, $\alpha_{1}(y)$ is to represent the position of $y$ based on the value of $\mathrm{in}_{1}$; similarly for $\alpha_{2}(z)$ and $\mathrm{in}_{2}$; and $\beta_{1}\left(y, y^{\prime}\right)$ is to decide whether $y$ and $y^{\prime}$ are consecutive positions or not. More specifically,

$-\alpha_{1}(y)=\exists y^{\prime}\left(\Pi_{S}\left(y, y^{\prime}\right) \wedge y \neq y^{\prime} \wedge \Pi_{P}(1, y)\right)$ if $\operatorname{in}_{1}=1$

$-\alpha_{1}(y)=\exists y^{\prime}\left(\Pi_{S}\left(y, y^{\prime}\right) \wedge y \neq y^{\prime} \wedge \Pi_{P}(0, y)\right)$ if in in $_{1}=0$; and

$-\alpha_{1}(y)=\Pi_{S}(y, y)$ if $\operatorname{in}_{i}=\epsilon$;

similarly for $\alpha_{2}(z)$. Furthermore,

$-\beta_{1}\left(y, y^{\prime}\right)=\Pi_{S}\left(y, y^{\prime}\right)$ if move $_{i}=+1$ and

$-\beta_{1}\left(y, y^{\prime}\right)=\left(y=y^{\prime}\right)$ if move $i=0$;

similarly for $\beta_{2}\left(z, z^{\prime}\right)$. That is, $\alpha_{1}(y)$ enforces $y$ to be a position in the string coded by $\Pi_{P}(1, y)$ or $\Pi_{P}(0, y)$ that has a successor, unless $y$ is the final position, where $\alpha_{1}(y)$ demands $\Pi_{S}(y, y)$; similarly for $\alpha_{2}(z)$. Moreover, $\beta_{1}\left(y, y^{\prime}\right)$ ensures that $y$ and $y^{\prime}$ are consecutive positions when $\mathcal{A}$ makes a move (with head 1) and $y=y^{\prime}$ otherwise; similarly for $\beta_{1}\left(z, z^{\prime}\right)$. Then, following Spielmann [2000], one can show that $\Phi=\exists y_{1} y_{2}\left[\mathrm{TC}_{x, y, z ; x^{\prime}, y^{\prime}, z^{\prime}} \bigvee_{\delta \in \Delta} \varphi_{\delta}\right]\left(s_{0}, 0,0, s_{a c c}, y_{1}, y_{2}\right)$ is satisfiable if and only if $L(\mathcal{A}) \neq \emptyset$.

Clearly, we can compute $\Phi$ using a query $\Pi_{\Phi}$ in FP. Recall that we still need to ensure the existence of an initial and a final position in well-formed instances of $I_{S}$. The final FP-query $\Pi$ is therefore defined as $\Pi_{\Phi} \wedge \Pi_{\text {ini }} \wedge \Pi_{\text {fin }}$, where $\Pi_{\text {ini }}=\exists w x \Pi_{S}(w, 0, x)$ and $\Pi_{\text {fin }}=\exists x \Pi_{S}(1, x, x)$.

This concludes the construction of $\mathcal{R}, \Pi$, and $\Theta$. One can verify that $L(\mathcal{A})$ is nonempty if and only if there exists an instance $\mathcal{I}$ of $\mathcal{R}$ such that $\mathcal{I} \models \Theta$ and $\Pi(\mathcal{I}) \neq \varnothing$. Note that the set $\Theta$ of FDs is fixed, independent of the 2-head DFA $L(\mathcal{A})$.

In light of Lemma 4.6, we show that $\operatorname{RCQP}^{s}(\mathrm{FP})$ is undecidable by reduction from the satisfiability problem for FP in the presence of FDs. Given an FP query $p(\vec{z})$ and a fixed set $\Theta$ of FDs, we construct a database schema $\mathcal{R}^{\prime}$, master data $D_{m}$, a set $V$ of 
CCs, and a FP query $Q$, such that $p$ is satisfiable in the presence of $\Theta$ if and only if $\operatorname{RCQ}^{s}\left(Q, D_{m}, V\right)$ is empty.

Suppose that $p$ and $\Theta$ are defined over a database schema $\mathcal{R}$. By Lemma 3.2, we assume without loss of generality that $\mathcal{R}$ consists of a single relation schema $R\left(A_{1}, \ldots, A_{m}\right)$.

(a) We define a database schema $\mathcal{R}^{\prime}=\left(R^{\prime}, E\right)$, where $R^{\prime}\left(G, A_{1}, \ldots, A_{m}\right)$ extends $R$ by adding a new attribute $G$ with an infinite domain, and $E(C)$ is a unary relation that consists of a single attribute $C$ with an infinite domain.

(b) We define CCs as follows. Note that, for each FD $X \rightarrow A$ in $\Theta,(G, X) \rightarrow A$ is an FD defined over $R^{\prime}$. Denote by $\Theta^{\prime}$ the set of all such FDs over $R^{\prime}$ deduced from FDs in $\Theta$. For each FD $(G, X) \rightarrow A$ in $\Theta^{\prime}$, we express it as a CC: $p_{v}\left(R^{\prime}\right) \subseteq \emptyset$, where

$$
p_{v}(g)=\exists \vec{x}, y, y^{\prime}, \vec{z}_{1}, \vec{z}_{2}\left(R^{\prime}\left(g, \vec{x}, y, \vec{z}_{1}\right) \wedge R^{\prime}\left(g, \vec{x}, y^{\prime}, \vec{z}_{2}\right) \wedge y \neq y^{\prime}\right),
$$

$\vec{x}, y$ and $\vec{z}_{1}$ correspond to attributes $X, A$, and $R^{\prime} \backslash(X \cup\{A\})$, respectively; similarly for $\vec{x}, y^{\prime}$ and $\vec{z}_{2}$. That is, $p_{v}\left(R^{\prime}\right)$ extracts tuples that violate the $\mathrm{FD}(G, X) \rightarrow A$. We define $V$ to be the set of all CCs constructed from FDs in $\Theta^{\prime}$ as delineated earlier. Intuitively, we group tuples of $R^{\prime}$ by the attribute $G$, such that FDs in $\Theta$ are imposed on each group individually. By Lemma 4.6, $\Theta$ is fixed; hence, so is $V$.

(c) The master data $D_{m}$ is assumed to be an empty relation $\emptyset$.

(d) We define $Q$ as follows. We first construct a query $p^{\prime}$ by substituting $R^{\prime}(g, \vec{y})$ for each occurrence of $R(\vec{y})$ in each rule of $p$, where $g$ is a variable corresponding to attribute $G$, and is shared across all the rules in $p^{\prime}$. One can verify that the following are equivalent:

- there exists an instance $I$ of $R$ such that $I \models \Theta$ and $p(I)$ is nonempty,

- there exists an instance $I^{\prime}$ of $R^{\prime}$ such that there exists $g \in \operatorname{dom}(G), I_{g}^{\prime} \models \Theta$ and $p\left(I_{g}^{\prime}\right)$ is nonempty, where $I_{g}^{\prime}$ is the subset of $I^{\prime}$ consisting of tuples $t$ with $t[G]=g$.

We define $Q(x):-E(x), p^{\prime}(g, \vec{y})$, that is, $Q\left(I^{\prime}, E\right)$ returns the $E$ relation if there exists $g$ such that $I_{g}^{\prime}=\Theta$ and $p\left(I_{g}^{\prime}\right)$ is nonempty.

We next show that $p$ is satisfiable in the presence of $\Theta$ if and only if $\operatorname{RCQ}^{s}\left(Q, D_{m}, V\right)$ is empty.

$\Rightarrow$ First, assume that $p$ is not satisfiable in the presence of $\Theta$, that is, there exists no instance $I$ of $R$ such that $I \models \Theta$ and $p(I)$ is nonempty. Then, $(\emptyset, \varnothing)$ is in $\operatorname{RCQ}^{s}\left(Q, D_{m}, V\right)$, that is, the empty instance of $R^{\prime}$ and the empty instance of $E$ make a database that is complete for $Q$ relative to $\left(D_{m}, V\right)$. For all instances $\left(I^{\prime}, E\right)$, if there exists $g \in \operatorname{dom}(G)$ such that $I_{g}^{\prime}=\Theta$, then $p\left(I_{g}^{\prime}\right)$ is empty; hence, $Q\left(I^{\prime}, E\right)=\emptyset$.

$\Leftarrow$ Conversely, assume that $p$ is satisfiable. Then, there exists an instance $I$ of $R$ such that $I \models \Theta$ and $p(I)$ is nonempty. We next show that $\operatorname{RCQ}^{s}\left(Q, D_{m}, V\right)$ is empty. That is, we need to prove that, for each instance $\left(I^{\prime}, E\right)$ of $\mathcal{R}^{\prime},\left(I^{\prime}, E\right) \notin \operatorname{RCQ}^{s}\left(Q, D_{m}, V\right)$ when $\left(\left(I^{\prime}, E\right), D_{m}\right) \models V$. We construct an extension $I^{\prime \prime}$ of $I^{\prime}$ such that, for each tuple $t$ in $I,(g, t)$ is in $I^{\prime \prime}$, for a constant $g \in \operatorname{dom}(G)$ that does not appear in the $G$ column of $I^{\prime}$. Let $E^{\prime}$ be an extension of $E$. Obviously, $\left(I^{\prime \prime}, E^{\prime}\right)$ is partially closed since $\left(I^{\prime}, E\right)$ is partially closed, $I_{g}^{\prime \prime} \models \Theta$, and the CCs in $V$ apply to tuples with the same $G$-attribute value. Furthermore, $p^{\prime}\left(I^{\prime \prime}\right)$ is nonempty. Hence, $\left(I^{\prime \prime}, E^{\prime}\right)$ is a partially closed extension of $\left(I^{\prime}, E\right)$. However, $Q\left(I^{\prime \prime}, E^{\prime}\right)=E^{\prime} \neq E=Q\left(I^{\prime}, E\right)$. Hence, $\left(I^{\prime}, E\right)$ is not in $\operatorname{RCQ}^{s}\left(Q, D_{m}, V\right)$.

(3) When $\mathcal{L}_{Q}$ is CQ, UCQ, or $\exists \mathrm{FO}^{+}$. It is known [Fan and Geerts 2010b] that RCQP $\left(\exists \mathrm{FO}^{+}\right)$is in NEXPTIME and that RCQP ${ }^{s}(\mathrm{CQ})$ is NEXPTIME-hard when $D_{m}$ and $V$ are fixed. 
From this and Lemma 4.4, it follows that $\operatorname{RCQP}\left(\mathcal{L}_{Q}\right)$ is NExPTIME-complete when $\mathcal{L}_{Q}$ is $\mathrm{CQ}, \mathrm{UCQ}$, or $\exists \mathrm{FO}^{+}$.

Note that, in these proofs, only fixed master data $D_{m}$ and fixed CCs are used. Hence, the complexity bounds remain intact when $V$ and $D_{m}$ are fixed.

\subsection{The Minimality Problem in the Strong Model}

This problem is to decide whether a database is relatively complete and does not contain excessive data. The following lemma tells us how to check this when ground instances are concerned.

Lemma 4.7. For every ground instance $I$, query $Q$, master data $D_{m}$, and set $V$ of $C C s$, (a) if $\left(I, D_{m}\right) \models V$, then, for every $I^{\prime} \subsetneq I$, $\left(I^{\prime}, D_{m}\right) \models V$; and (b) if $I$ is in $\operatorname{RCQ}^{s}\left(Q, D_{m}, V\right)$, then $I$ is not minimal if and only if there exists a tuple $t \in I$ such that $I \backslash\{t\} \in \mathrm{RCQ}^{s}\left(Q, D_{m}, V\right)$.

Proof. Consider query $Q$, master data $D_{m}$, and a set $V$ of CCs. By Lemma 3.2, we assume without loss of generality that $Q$ is defined over a single relation schema $R$. Given an instance $I$ of $R$, we show the following.

(a) If $\left(I, D_{m}\right) \models V$, then, for every $I^{\prime} \subseteq I,\left(I^{\prime}, D_{m}\right) \models V$. We show that, for every $\phi \in V$, $\left(I^{\prime}, D_{m}\right) \models \phi$. Let $\phi$ be $q(R) \subseteq p\left(D_{m}\right)$, where $q$ is a CQ query. Since $I^{\prime} \subseteq I, q\left(I^{\prime}\right) \subseteq q(I)$ because CQ queries are monotonic. By $\left(I, D_{m}\right) \models V, q(I) \subseteq p\left(D_{m}\right)$; hence, $q\left(I^{\prime}\right) \subseteq \bar{p}\left(D_{m}\right)$, that is, $\left(I^{\prime}, D_{m}\right) \models \phi$.

(b) Suppose that $I$ is in $\operatorname{RCQ}^{s}\left(Q, D_{m}, V\right)$. Then, $I$ is not minimal if and only if there exists a tuple $t \in I$ such that $I \backslash\{t\} \in \mathrm{RCQ}^{s}\left(Q, D_{m}, V\right)$. To see this, first assume that $I \backslash\{t\} \in \mathrm{RCQ}^{s}\left(Q, D_{m}, V\right)$. Then, obviously, $I$ is not minimal by the definition of minimal instances. Conversely, suppose that $I$ is not minimal, that is, there exists $I_{1} \subsetneq I$ such that for each $I_{2} \in \operatorname{Ext}\left(I_{1}, D_{m}, V\right), Q\left(I_{1}\right)=Q\left(I_{2}\right)$. Note that $I \in \operatorname{Ext}\left(I_{1}, D_{m}, V\right)$. Then, there must exist $I_{2}=I \backslash\{t\}$ for some $t \in I$ such that $I_{1} \subseteq I_{2}$ and $Q\left(I_{1}\right)=Q\left(I_{2}\right)$. By (a), $\left(I_{2}, D_{m}\right) \models V$. In addition, for all $I^{\prime} \in \operatorname{Ext}\left(I_{2}, D_{m}, V\right), I^{\prime}$ is also in $\operatorname{Ext}\left(I_{1}, D_{m}, V\right)$; hence, $Q\left(I^{\prime}\right)=Q\left(I_{1}\right)=Q\left(I_{2}\right)$. Thus, $I_{2}$ is in $\operatorname{RCQ}^{s}\left(Q, D_{m}, V\right)$.

Capitalizing on this lemma, next, we provide complexity bounds on $\operatorname{MINP}^{s}\left(\mathcal{L}_{Q}\right)$. Here, the presence of missing values again makes the problem a little harder: $\operatorname{MINP}^{s}(C Q)$ is $\mathrm{D}_{2}^{p}$-complete for ground instances, but it is $\Pi_{3}^{p}$-complete for $c$-instances. Here, $\mathrm{D}_{2}^{p}$ is the class of languages recognized by oracle machines that make a call to a $\Sigma_{2}^{p}$ oracle and a call to a $\Pi_{2}^{p}$ oracle. That is, $L$ is in $D_{2}^{p}$ if there exist languages $L_{1} \in \Sigma_{2}^{p}$ and $L_{2} \in \Pi_{2}^{p}$ such that $L=L_{1} \cap L_{2}$ [Wooldridge and Dunne 2004].

TheOREM 4.8. When $\mathcal{L}_{Q}$ is $F O$ or $F P, \operatorname{MINP}^{s}\left(\mathcal{L}_{Q}\right)$ is undecidable both for ground instances and for c-instances. When $\mathcal{L}_{Q}$ is $C Q, U C Q$, or $\exists F O^{+}, \operatorname{MINP}^{s}\left(\mathcal{L}_{Q}\right)$ is

$-\Pi_{3}^{p}$-complete for c-instances, and

$-\mathrm{D}_{2}^{p}$-complete for ground instances.

The complexity is unchanged when $D_{m}$ and $V$ are fixed.

Proof. We first show that $\operatorname{MINP}^{s}\left(\mathcal{L}_{Q}\right)$ is undecidable when $\mathcal{L}_{Q}$ is either FO or FP. We then verify that, when $\mathcal{L}_{Q}$ is $\mathrm{CQ}, \mathrm{UCQ}$, or $\exists \mathrm{FO}^{+}, \mathrm{MINP}^{s}\left(\mathcal{L}_{Q}\right)$ is $\Pi_{3}^{p}$-complete for $c$ instances but is $\mathrm{D}_{2}^{p}$-complete for ground instances. In the proofs for undecidability and lower bounds to be given later, we use fixed $D_{m}$ and $V$.

(1) When $\mathcal{L}_{Q}$ is FO or FP. To show that $\operatorname{MINP}^{s}\left(\mathcal{L}_{Q}\right)$ is undecidable when $\mathcal{L}_{Q}$ is either FO or FP, it suffices to show that these problems are undecidable for ground instances, since ground instances are $c$-instances themselves. In addition, it suffices to show it 
is undecidable to determine, given a query $Q$, master data $D_{m}$, and a set of $V$ of CCs, whether a special instance $\mathcal{I}_{\emptyset}$ is in $\operatorname{RCQ}^{s}\left(Q, D_{m}, V\right)$, where $\mathcal{I}_{\emptyset}$ is the empty instance of the schema over which $Q$ is defined. If $\mathcal{I}_{\emptyset}$ is in $\operatorname{RCQ}^{s}\left(Q, D_{m}, V\right)$, then it is a minimal instance complete for $Q$ relative to $\left(D_{m}, V\right)$.

$\operatorname{MINP}^{s}(F O)$. We verify the undecidability of $\mathrm{MINP}^{s}(\mathrm{FO})$ by reduction from the satisfiability of FO queries. Given an FO query $q$, we assume without loss of generality by Lemma 3.2 that $q$ is defined over a single relation $R$. Consider the FO query $Q$ defined in the proof of Theorem 4.5 (1), and let $D_{m}$ and $V$ both be empty. It has been shown there that if $q$ is not satisfiable, then $I_{\emptyset}$ is in $\operatorname{RCQ}^{s}\left(Q, D_{m}, V\right)$. Conversely, if $q$ is satisfiable, then $\operatorname{RCQ}^{s}\left(Q, D_{m}, V\right)$ is empty; hence, $I_{\emptyset}$ is not in $\operatorname{RCQ}^{s}\left(Q, D_{m}, V\right)$. Thus, $q$ is satisfiable if and only if $I_{\emptyset}$ is minimal in $\operatorname{RCQ}^{s}\left(Q, D_{m}, V\right)$.

$\operatorname{MINP}^{s}(F P)$. Along the same lines as the proof for $\mathrm{MINP}^{s}(\mathrm{FO})$, it suffices to show that, given $Q$ in FP, $D_{m}$, and $V$, it is undecidable to determine whether the special instance $I_{\emptyset}$ is in $\operatorname{RCQ}^{s}\left(Q, D_{m}, V\right)$. This has already been verified by the proof of Theorem $4.5(2)$. It has been shown that deciding nonemptiness of $\operatorname{RCQ}^{s}\left(Q, D_{m}, V\right)$ is undecidable. In particular, $\operatorname{RCQ}^{s}\left(Q, D_{m}, V\right) \neq \emptyset$ if and only if $I_{\emptyset} \in \mathrm{RCQ}^{s}\left(Q, D_{m}, V\right)$.

(2) When $\mathcal{L}_{Q}$ is $\mathrm{CQ}, \mathrm{UCQ}$, or $\exists \mathrm{FO}^{+}$. We prove that $\operatorname{MINP}^{s}\left(\mathcal{L}_{Q}\right)$ is $\Pi_{3}^{p}$-complete for $c$ instances and $D_{2}^{p}$-complete for ground instances.

(2.1) For $c$-instances. To show that $\operatorname{MINP}^{s}\left(\mathcal{L}_{Q}\right)$ is $\Pi_{3}^{p}$-complete for $c$-instances when $\mathcal{L}_{Q}$ is $\mathrm{CQ}, \mathrm{UCQ}$, or $\exists \mathrm{FO}^{+}$, it suffices to verify that $\mathrm{MINP}^{s}(\mathrm{CQ})$ is $\Pi_{3}^{p}$-hard and that $\operatorname{MINP}^{s}\left(\exists \mathrm{FO}^{+}\right)$is in $\Pi_{3}^{p}$.

Lower bound. We show that $\mathrm{MINP}^{s}(\mathrm{CQ})$ is $\Pi_{3}^{p}$-hard by reduction from the complement of the $\exists^{*} \forall^{*} \exists^{*} 3$ sAT problem, which is known to be $\Sigma_{3}^{p}$-complete (see Papadimitriou [1994]). The $\exists^{*} \forall^{*} \exists^{*} 3$ sAT problem is to determine, given a sentence $\varphi=\exists X \forall Y \exists Z \psi$, whether or not $\varphi$ is true. Here, $X=\left\{x_{1}, \ldots, x_{n}\right\}, Y=\left\{y_{1}, \ldots, y_{m}\right\}, Z=\left\{z_{1}, \ldots, z_{k}\right\}$, and $\psi$ is an instance of 3sAT.

Given an instance $\varphi=\exists X \forall Y \exists Z \psi$ of the $\exists^{*} \forall^{*} \exists^{*}$ 3sAT problem, we define a database schema $\mathcal{R}$, a $c$-instance $\mathcal{T}$ of $\mathcal{R}$, master data $D_{m}$, a set $V$ of CCs, and a query $Q$ in $\mathrm{CQ}$, such that $\varphi$ is true if and only if $\mathcal{T}$ is not a minimal $c$-instance in $\operatorname{RCQ}^{s}\left(Q, D_{m}, V\right)$.

(a) The database schema $\mathcal{R}$ consists of six relation schemas: $R_{(0,1)}(A), R_{\neg}(A, \bar{A})$, $R_{\vee}\left(A_{1}, A_{2}, B\right), R_{\wedge}\left(A_{1}, A_{2}, B\right), R_{X}(\mathrm{id}, X)$, and $R_{s}(W)$, where $R_{(0,1)}, R_{\neg}, R_{\vee}$, and $R_{\wedge}$ are the same as their counterparts in the proof of Proposition 3.3. The relation $R_{X}(\mathrm{id}, X)$ is to encode a truth assignment for variables in $X ; R_{s}(W)$ is used to inspect query answers, as will become clear shortly.

(b) We construct a $c$-instance $\mathcal{T}=\left(I_{(0,1)}, I_{\neg}, I_{\vee}, I_{\wedge}, T_{X}, I_{s}\right)$, in which $I_{(0,1)}, I_{\neg}, I_{\vee}, I_{\wedge}$ are ground relations, as shown in Figure 2 , while $T_{X}=\left(\left\{\left(1, x_{1}\right),\left(2, x_{2}\right), \ldots,\left(n, x_{n}\right)\right\}\right.$, true $)$ is a $c$-table, consisting of the variables in $X$, without any local condition. Finally, $I_{s}$ consists of two tuples, (0) and (1). Intuitively, we use $\mathcal{T}$ to encode basic Boolean operations, truth assignments for variables of $X$, and possible truth values of $\psi$ in the reduction.

(c) Master data $D_{m}$ is specified by five relation schemas: $R_{(0,1)}^{m}=R_{(0,1)}, R_{\neg}^{m}=R_{\neg}$, $R_{\vee}^{m}=R_{\vee}, R_{\wedge}^{m}=R_{\wedge}$, and $R_{\emptyset}^{m}(X)$. The master data instances consist of $I_{(0,1)}^{m}=I_{(0,1)}$, $I_{\neg}^{m}=I_{\neg}, I_{\vee}^{m}=I_{\vee}, I_{\wedge}^{m}=I_{\wedge}$, and $I_{\emptyset}^{m}=\emptyset$.

(d) The set $V$ consists of the following CCs:

$-R_{(0,1)} \subseteq R_{(0,1)}^{m}, R_{\neg} \subseteq R_{\neg}^{m}, R_{\vee} \subseteq R_{\vee}^{m}, R_{\wedge} \subseteq R_{\wedge}^{m}$, and $R_{s} \subseteq R_{(0,1)}^{m} ;$

$-\exists \mathrm{id} R_{X}(\mathrm{id}, x) \subseteq R_{(0,1)}^{m}(x)$; and

$-q_{\text {id }}(x) \subseteq R_{\emptyset}^{m}(x)$, where $q_{\text {id }}(x)=\exists y, y^{\prime} R_{X}(x, y) \wedge R_{X}\left(x, y^{\prime}\right) \wedge\left(y \neq y^{\prime}\right)$. This is to ensure that id is a key for $R_{X}$. 
The last two CCs ensure that each instance of $R_{X}$ is indeed a truth assignment of variables in $X$.

(e) We next define the query $Q$, such that $\varphi$ is true if and only if (i) there exists a ground instance $\mathcal{I}$ of $\mathcal{T}$ that encodes a truth assignment $\mu_{X}$ of $X$ variables by an instance of $T_{X}$ in $\mathcal{I}$, and (ii) $Q(\mathcal{I})$ returns tuples representing all truth assignments $\mu_{Y}$ of $Y$ variables when $\psi$ is true under $\left(\mu_{X}, \mu_{Y}, \mu_{Z}\right)$; here, $\mu_{Z}$ is a truth assignment of $Z$ variables, which is encoded by a tuple returned by a subquery of $Q$ on $\mathcal{I}$ (if it exists). More specifically, query $Q$ is defined as follows.

$$
Q(\vec{y})=\exists \vec{x}, \vec{z}\left(Q_{X}(\vec{x}) \wedge Q_{Y}(\vec{y}) \wedge Q_{Z}(\vec{z}) \wedge Q_{\psi}(\vec{x}, \vec{y}, \vec{z}, w) \wedge R_{s}(w) \wedge Q_{\mathrm{all}}\right),
$$

where $Q_{X}$ is a CQ query $Q_{X}\left(x_{1}, \ldots, x_{n}\right)=\bigwedge_{i \in[1, n]} R_{X}\left(i, x_{i}\right)$, that is, it selects from $R_{X}$ the truth assignments for $X$. The query $Q_{Y}(\vec{y})=R_{(0,1)}\left(y_{1}\right) \wedge \cdots \wedge R_{(0,1)}\left(y_{m}\right)$ constructs all possible truth assignments of variables in $Y$; similarly for $Q_{Z}(\vec{z})$ and $Z$. Given a truth assignment $\left(\mu_{X}, \mu_{Y}, \mu_{Z}\right)$ of $(X, Y, Z)$, the subquery $Q_{\psi}\left(\mu_{X}, \mu_{Y}, \mu_{Z}, w\right)$ is to evaluate $\psi\left(\mu_{X}, \mu_{Y}, \mu_{Z}\right)$, and it records its truth value in $w$, which is either 0 or 1 . Obviously, $Q_{\psi}$ can be defined in CQ by leveraging relations $I_{\neg}, I_{\vee}$, and $I_{\wedge}$. The query $Q_{\text {all }}$ is to ensure that all the tuples in $I_{(0,1)}, I_{\neg}, I_{\vee}, I_{\wedge}$, and tuple (1) in $I_{s}$ are in place. More specifically, it is defined as

$$
Q_{\mathrm{all}}=Q_{(0,1)} \wedge Q_{\neg} \wedge Q_{\vee} \wedge Q_{\wedge} \wedge Q_{s},
$$

where $Q_{\vee}=R_{\vee}(0,0,0) \wedge R_{\vee}(0,1,1) \wedge R_{\vee}(1,0,1) \wedge R_{\vee}(1,1,1)$ asserts that the removal of any of the four tuples in $I_{\vee}$ makes $Q(\vec{y})$ empty; similarly for $Q_{(0,1)}, Q_{\neg}$, and $Q_{\wedge}$. In addition, $Q_{s}=R_{s}(1)$, asserting that the removal of (1) makes $Q(\vec{y})$ empty. Intuitively, query $Q$ returns all tuples encoding truth assignments $\mu_{Y}$ of $Y$ such that, for the truth assignment $\mu_{X}$ of $X$ encoded by $T_{X}, \exists Z \psi\left(\mu_{X}, \mu_{Y}, Z\right)$ evaluates to a truth value in $I_{s}$.

We show that $\varphi$ is false if and only if $\mathcal{T}$ is a minimal $c$-instance in $\operatorname{RCQ}^{s}\left(Q, D_{m}, V\right)$, that is, for each ground instance $\mathcal{I}$ of $\mathcal{T}, \mathcal{I}$ is minimal in $\operatorname{RCQ}^{s}\left(Q, D_{m}, V\right)$. The argument is based on the following observations: for every ground instance $\mathcal{I}=$ $\left(I_{(0,1)}, I_{\wedge}, I_{\vee}, I_{\neg}, I_{X}, I_{s}\right)$ of $\mathcal{T}$, (i) $\mathcal{I}$ has no extensions by the definition of $V$, that is, $V$ enforces an upper limit on the potential valuations of $\mathcal{T}$; (ii) removing any tuple from $I_{X}, I_{(0,1)}, I_{\neg}, I_{\vee}$, or $I_{\wedge}$, or removing tuple (1) from $I_{s}$ would make $Q$ empty, by the definitions of $Q$ and $Q_{\text {all }}$; that is, $Q_{\text {all }}$ imposes a lower limit on which tuples must exist.

$\Rightarrow$ First, assume that $\varphi$ is false. Then, for each truth assignment $\mu_{X}$ of $X$, there exists a truth assignment $\mu_{Y}$ of $Y$ such that $\exists Z \psi\left(\mu_{X}, \mu_{Y}, Z\right)$ is false. It is easy to see that, for all ground instances $\mathcal{I}=\left(I_{(0,1)}, I_{\wedge}, I_{\vee}, I_{\neg}, I_{X}, I_{s}\right) \in \operatorname{Mod}(\mathcal{T})$ of $\mathcal{T}, Q(\mathcal{I})$ returns all truth assignments of $Y$ since $I_{s}$ consists of $(0)$ and (1). As argued in (i) earlier, there exists no extension to $\mathcal{I}$; thus, $\mathcal{I} \in \mathrm{RCQ}^{s}\left(Q, D_{m}, V\right)$. We next show that $\mathcal{I}$ is minimal. As argued in (ii) earlier, we only need to consider the instance $\mathcal{I}^{\prime}=\left(I_{(0,1)}, I_{\wedge}, I_{\vee}, I_{\neg}, I_{X}, I_{s}^{\prime}\right)$, where $I_{s}^{\prime}=\{(1)\}$. Obviously, $Q\left(\mathcal{I}^{\prime}\right)$ does not contain all truth assignments of $Y$ since $\varphi$ is false, making it different from $Q(\mathcal{I})$. Hence, $\mathcal{I}$ is a minimal $c$-instance.

$\Leftarrow$ Conversely, suppose that $\varphi$ is true. Then, there exists a truth assignment $\mu_{X}^{0}$ of $X$ such that, for every truth assignment $\mu_{Y}$ of $Y, \exists Z \psi\left(\mu_{X}^{0}, \mu_{Y}, Z\right)$ is true. We show that there exists a ground instance in $\operatorname{Mod}\left(T, D_{m}, V\right)$ that is not minimal. Let $I_{X}^{0}$ be a ground instance of $T_{X}$ that agrees with $\mu_{X}^{0}$, and $\mathcal{I}^{0}=\left(I_{(0,1)}, I_{\wedge}, I_{\vee}, I_{\neg}, I_{X}^{0}, I_{s}\right)$. As before, $Q\left(\mathcal{I}^{0}\right)$ consists of all truth assignments of $Y$. However, $\mathcal{I}^{0}$ is not minimal. Consider $\mathcal{I}^{\prime}=\left(I_{(0,1)}, I_{\wedge}, I_{\vee}, I_{\neg}, I_{X}^{0}, I_{s}^{\prime}\right)$ with $I_{s}^{\prime}=\{(1)\}$. Then, since $\varphi$ is true and $I_{X}^{0}$ encodes $\mu_{X}^{0}, Q\left(\mathcal{I}^{\prime}\right)$ consists again of all truth assignments of $Y$. Hence, $\mathcal{I}^{0}$ is not minimal; thus, $\mathcal{T}$ is not a minimal $c$-instance in $\operatorname{RCQ}^{s}\left(Q, D_{m}, V\right)$. 
Upper bound. We next show that $\mathrm{MINP}^{s}\left(\exists \mathrm{FO}^{+}\right)$is in $\Pi_{3}^{p}$ for $c$-instances. The proof makes use of Lemma 4.7 and the $\Sigma_{2}^{p}$ algorithm [Fan and Geerts 2009] for checking whether a ground instance is not in $\mathrm{RCQ}^{s}\left(Q, D_{m}, V\right)$.

We give an $\Sigma_{3}^{p}$ algorithm for the complement of $\operatorname{MINP}^{s}\left(\exists \mathrm{FO}^{+}\right)$: given a $c$-instance $\mathcal{T}$, a query $Q$ in $\exists \mathrm{FO}^{+}$, master data $D_{m}$, and a set $V$ of CCs, the algorithm returns "yes" if and only if $\mathcal{T}$ is not a minimal $c$-instance in $\operatorname{RCQ}^{s}\left(Q, D_{m}, V\right)$. By Lemma 3.2, we assume without loss of generality that $\mathcal{T}$ consists of a single $c$-table $T$. Assume that $T$ consists of $k$ tuples $\tau_{1}, \ldots, \tau_{k}$. Recall the notion of Adom given in the proof of Theorem 4.1. The algorithm works as follows.

(1) Guess a valuation $\mu$ of $(T, \xi)$ with Adom and guess an index $i \in[1, k]$. Let $I=\mu(T)$.

(2) Test the following:

(a) whether $I \notin \operatorname{Mod}\left(T, D_{m}, V\right)$; if not, continue; otherwise, reject the current guess;

(b) whether $I$ is not a complete ground instance; if so, return "yes";

(c) whether $\mu\left(\tau_{i}\right) \in I$ and $I \backslash \mu\left(\tau_{i}\right)$ is in $\operatorname{RCQ}^{s}\left(Q, D_{m}, V\right)$. If so, return "yes"; otherwise, reject the current guess.

The algorithm is in $\Sigma_{3}^{p}$. It invokes an NP oracle to check whether an instance is in $\operatorname{Mod}\left(T, D_{m}, V\right)$ in Step (2)(a), invokes an $\Sigma_{2}^{p}$ oracle to check whether $I \notin \operatorname{RCQ}^{s}\left(Q, D_{m}, V\right)$ in Step (2)(b), and a $\Pi_{2}^{p}$ oracle to check whether $I \backslash \mu\left(\tau_{i}\right) \in \mathrm{RCQ}^{s}\left(Q, D_{m}, B\right)$ (see the proof of Theorem 4.1). Hence, the algorithm is in NP $\Sigma_{2}^{p}$, that is, in $\Sigma_{3}^{p}$.

The algorithm returns "yes" when a counterexample is found that dispels $T$ as a minimal $c$-instance in $\operatorname{RCQ}^{s}\left(Q, D_{m}, V\right)$. Conversely, suppose that $T$ is not a minimal $c$-instance in $\operatorname{RCQ}^{s}\left(Q, D_{m}, V\right)$. Then, along the same lines as the proofs of Lemmas 4.2 and 4.3 and the upper bound proof of Theorem 4.1 for $\operatorname{RCDP}^{s}\left(\exists \mathrm{FO}^{+}\right)$, one can show that there must be a ground instance $I \in \operatorname{Mod}_{\text {Adom }}\left(T, D_{m}, V\right)$ such that $I$ is not a minimal ground instance in $\operatorname{RCQ}^{s}\left(Q, D_{m}, V\right)$, that is, either $I$ is not in $\operatorname{RCQ}^{s}\left(Q, D_{m}, V\right)$ or $I$ is in $\operatorname{RCQ}^{s}\left(Q, D_{m}, V\right)$, but there exists $I^{\prime} \subsetneq I$ such that $I^{\prime}$ is also in $\operatorname{RCQ}^{s}\left(Q, D_{m}, V\right)$. The former is checked by the $\Sigma_{2}^{p}$ oracle in Step (2)(b). The latter is inspected by Step (2)(c) of the algorithm, which tests whether there exists a tuple $t=\mu\left(\tau_{i}\right)$ in $I$ such that $I \backslash\{t\}$ is in $\operatorname{RCQ}^{s}\left(Q, D_{m}, V\right)$. This suffices by Lemma 4.7. Hence, the algorithm is able to find a counterexample $I$ and, hence, returns "yes."

(2.2) For ground instances. When it comes to ground instances, we show that $\operatorname{MINP}^{s}\left(\mathcal{L}_{Q}\right)$ is $\mathrm{D}_{2}^{p}$-complete, when $\mathcal{L}_{Q}$ is $\mathrm{CQ}, \mathrm{UCQ}$, or $\exists \mathrm{FO}^{+}$. It suffices to prove that, in this setting, $\operatorname{MINP}(C Q)$ is $\mathrm{D}_{2}^{p}$-hard and that $\operatorname{MINP}^{s}\left(\exists \mathrm{FO}^{+}\right)$is in $\mathrm{D}_{2}^{p}$.

Lower bound. We first show that $\mathrm{MINP}^{s}(\mathrm{CQ})$ is $\mathrm{D}_{2}^{p}$-hard by reduction from the $\exists^{*} \forall^{*} 3 \mathrm{DNF}$ $\forall^{*} \exists^{*} 3 \mathrm{CNF}$ problem, which is $\mathrm{D}_{2}^{p}$-complete [Wooldridge and Dunne 2004]. An instance of $\exists^{*} \forall^{*} 3$ DNF $-\forall^{*} \exists^{*}$ CNF is a pair of $\forall^{*} \exists^{*} 3$ SAT instances $\varphi_{1}=\forall X_{1} \exists Y_{1} \psi_{1}\left(X_{1}, Y_{1}\right)$ and $\varphi_{2}=\forall X_{2} \exists Y_{2} \psi_{2}\left(X_{2}, Y_{2}\right)$. It is to decide whether $\varphi_{1}$ is true and $\varphi_{2}$ is false. Given $\left(\varphi_{1}, \varphi_{2}\right)$, we define $\mathcal{R}, \mathcal{I}, D_{m}, V$, and $Q$, such that $\varphi_{1}$ is true and $\varphi_{2}$ is false if and only if $\mathcal{I}$ is a minimal instance in $\operatorname{RCQ}^{s}\left(Q, D_{m}, V\right)$.

(a) The database schema $\mathcal{R}$ consists of six relation schemas: $R_{(0,1)}(A), R_{\neg}(A, \bar{A})$, $R_{\vee}\left(A_{1}, A_{2}, B\right)$, and $R_{\wedge}\left(A_{1}, A_{2}, B\right)$, the same as their counterparts in the proof of Proposition 3.3, as well as $R_{1}\left(W_{1}\right)$ and $R_{2}\left(W_{2}\right)$ to encode relations that will be used to select appropriate truth values, as will be detailed later.

(b) We construct a ground instance $\mathcal{I}=\left(I_{(0,1)}, I_{\neg}, I_{\vee}, I_{\wedge}, I_{1}, I_{2}\right)$, in which $I_{(0,1)}, I_{\neg}, I_{\vee}$, and $I_{\wedge}$ are ground relations, as shown in Figure $2, I_{1}=\{(1)\}$ and $I_{2}=\{(0),(1)\}$. 
(c) Master data $D_{m}$ is specified by six relation schemas: $R_{(0,1)}^{m}=R_{(0,1)}, R_{\neg}^{m}=R_{\neg}$, $R_{\vee}^{m}=R_{\vee}, R_{\wedge}^{m}=R_{\wedge}, R_{1}^{m}=R_{1}$, and $R_{2}^{m}=R_{2}$. The master data instance consists of $I_{(0,1)}^{m}=I_{(0,1)}, I_{\neg}^{m}=I_{\neg}, I_{\vee}^{m}=I_{\vee}, I_{\wedge}^{m}=I_{\wedge}$, and $I_{1}^{m}=I_{2}^{m}=\{(0),(1)\}$.

(d) The set $V$ consists of the following CCs: $R_{(0,1)} \subseteq R_{(0,1)}^{m}, R_{\neg} \subseteq R_{\neg}^{m}, R_{\vee} \subseteq R_{\vee}^{m}, R_{\wedge} \subseteq R_{\wedge}^{m}$, $R_{1} \subseteq R_{1}^{m}$, and $R_{2} \subseteq R_{2}^{m}$.

(e) The CQ query $Q\left(\vec{x}_{1}, \vec{x}_{2}\right)$ is defined as $Q_{1}\left(\vec{x}_{1}\right) \wedge Q_{2}\left(\vec{x}_{2}\right) \wedge Q_{\text {all }}$, where $\vec{x}_{1}$ and $\vec{x}_{2}$ correspond to the $X$-variables in $\varphi_{1}$ and $\varphi_{2}$, respectively. The query $Q_{\text {all }}$ is used to ensure that (i) all tuples in $I_{01}, I_{\neg}, I_{\vee}$, and $I_{\wedge}$ are present; and (ii) that $I_{1}$ and $I_{2}$ contain (1). Otherwise, $Q_{\text {all }}$ returns false (empty set). The queries $Q_{i}\left(\vec{x}_{i}\right)$ for $i=1,2$ are defined as

$$
Q_{i}\left(\vec{x}_{i}\right)=\exists \vec{y}_{i}, w_{i}\left(Q_{X_{i}}\left(\vec{x}_{i}\right) \wedge Q_{Y_{i}}(\vec{y}) \wedge Q_{\psi_{i}}\left(\vec{x}_{i}, \vec{y}_{i}, w_{i}\right) \wedge R_{i}\left(w_{i}\right)\right),
$$

where $Q_{X_{i}}$ (resp. $Q_{Y_{i}}$ ) generates all truth assignments for $X_{i}$ (resp. $Y_{i}$ ) by means of Cartesian products of $R_{01}$; and $Q_{\psi_{i}}$ is a CQ query encoding $\psi_{i}$ by leveraging relations $I_{\neg}, I_{\vee}$, and $I_{\wedge}$. More specifically, for given truth assignments $\mu_{X_{i}}$ and $\mu_{Y_{i}}$ of $X_{i}$ and $Y_{i}$, respectively, $Q_{\psi_{i}}\left(\mu_{X_{i}}, \mu_{Y_{i}}, 0\right)$ is true if $\psi_{i}\left(\mu_{X_{i}}, \mu_{Y_{i}}\right)$ is false, and $Q_{\psi_{i}}\left(\mu_{X_{i}}, \mu_{Y_{i}}, 1\right)$ is true if $\psi_{i}\left(\mu_{X_{i}}, \mu_{Y_{i}}\right)$ is true. The final conjunct in $Q_{i}$ controls what kind of truth values are returned. We consider the following cases: (a) If $I_{i}=\{(1)\}$, then $Q_{i}\left(\vec{x}_{i}\right)$ returns all truth assignments $\mu_{X_{i}}$ for $X_{i}$ for which there exists a truth assignment $\mu_{Y_{i}}$ of $Y_{i}$ that satisfies $\psi_{i}$; and (b) if $I_{i}=\{(0),(1)\}$, then $Q_{i}\left(\vec{x}_{i}\right)$ returns all possible truth assignments of $X_{i}$.

We next verify that $\varphi_{1}$ is true and $\varphi_{2}$ is false if and only if $\mathcal{I}$ is a minimal instance in $\operatorname{RCQ}\left(Q, D_{m}, V\right)$.

$\Rightarrow$ Suppose that $\varphi_{1}$ is true and $\varphi_{2}$ is false. Observe that $Q(\mathcal{I})=F_{X_{1}} \times F_{X_{2}}$, where $F_{X_{i}}$ consists of all possible truth assignments for $X_{i} . Q_{1}(\mathcal{I})=F_{X_{1}}$ because $\varphi_{1}$ is true, whereas $Q_{2}(\mathcal{I})=F_{X_{2}}$ because $I_{2}$ consists of both (0) and (1). Furthermore, observe that $\mathcal{I}$ is also complete. The only possible extension of $\mathcal{I}$ is $\mathcal{I}^{\prime}=\left(I_{(0,1)}, I_{\neg}, I_{\vee}, I_{\wedge}, I_{1}^{\prime}, I_{2}\right)$, with $I_{1}^{\prime}=\{(0),(1)\}$. Clearly, $Q\left(\mathcal{I}^{\prime}\right)=Q(\mathcal{I})$ since $Q(\mathcal{I})$ already generates the largest possible query result. That is, $\mathcal{I} \in \mathrm{RCQ}^{s}\left(Q, D_{m}, V\right)$. We next show that $\mathcal{I}$ is also minimal. For this, it suffices to observe that, among all possible subsets of $\mathcal{I}$, the instance $\mathcal{I}^{\prime \prime}=\left(I_{(0,1)}, I_{\neg}, I_{\vee}, I_{\wedge}, I_{1}, I_{2}^{\prime \prime}\right)$ with $I_{2}^{\prime \prime}=\{(1)\}$ is the only one that can possibly lead to $Q\left(\mathcal{I}^{\prime \prime}\right) \neq \emptyset$. All other subinstances of $\mathcal{I}$ make $Q_{\text {all }}$ return empty; thus, these cannot be complete because $Q(\mathcal{I}) \neq \emptyset$. It remains to show that $Q\left(\mathcal{I}^{\prime \prime}\right) \subsetneq Q(\mathcal{I})$, thus $\mathcal{I}^{\prime \prime}$ is not in $\operatorname{RCQ}^{s}\left(Q, D_{m}, V\right)$. To see this, recall that $\varphi_{2}$ is false. This implies that there exists a truth assignment $\mu_{X_{2}}^{0}$ of $X_{2}$ for which all truth assignments $\mu_{Y_{2}}$ of $Y_{2}$ make $\psi$ false. Since $I_{2}^{\prime \prime}=\{(1)\}, Q_{2}\left(\mathcal{I}^{\prime \prime}\right)$ will not return $\mu_{X_{2}}^{0}$. On the other hand, $\mu_{X_{2}}^{0} \in Q_{2}(\mathcal{I})$. Since $Q_{1}(\mathcal{I})=Q_{1}\left(\mathcal{I}^{\prime \prime}\right)$, we can conclude that $Q\left(\mathcal{I}^{\prime \prime}\right) \subsetneq Q(\mathcal{I})$.

$\Leftarrow$ Suppose that $\varphi_{1}$ is false or $\varphi_{2}$ is true. We distinguish between the following two cases: (i) $\varphi_{1}$ is false and (ii) both $\varphi_{1}$ and $\varphi_{2}$ are true. For case (i), we immediately have that $\mathcal{I}$ is not in $\operatorname{RCQ}^{s}\left(Q, D_{m}, V\right)$, thus cannot be minimal. Consider the unique extension $\mathcal{I}^{\prime}$ of $\mathcal{I}$ described earlier. Clearly, $Q\left(\mathcal{I}^{\prime}\right)=F_{X_{1}} \times F_{X_{2}}$. However, since $\varphi_{1}$ is false and $I_{1}$ contains only (1), $Q_{1}(\mathcal{I})$ will not include at least one truth assignment of $X_{1}$. Hence, $Q(\mathcal{I}) \subsetneq Q\left(\mathcal{I}^{\prime}\right)$. For case (ii), since $\varphi_{1}$ and $\varphi_{2}$ are both true, $Q(\mathcal{I})=F_{X_{1}} \times F_{X_{2}}$; thus, $Q\left(\mathcal{I}^{\prime}\right)=Q(\mathcal{I})$ since no more result tuples can be added. That is, $\mathcal{I}$ is in $\operatorname{RCQ}^{s}\left(Q, D_{m}, V\right)$. We show that $\mathcal{I}$ is not minimal. Consider the subinstance $\mathcal{I}^{\prime \prime}$ described earlier with $I_{2}^{\prime \prime}=\{(1)\}$. We claim that $\mathcal{I}^{\prime \prime}$ is in $\operatorname{RCQ}^{s}\left(Q, D_{m}, V\right)$. To see this, observe that the only extensions of $\mathcal{I}^{\prime \prime}$ are $\mathcal{I}, \mathcal{I}^{\prime}$, and $\mathcal{I}^{\prime \prime \prime}=\left(I_{(0,1)}, I_{\neg}, I_{\vee}, I_{\wedge}, I_{1}^{\prime}, I_{2}^{\prime \prime}\right)$. Since $\varphi_{1}$ is true, adding $(0)$ to $I_{1}$ (as done in the extensions $\mathcal{I}^{\prime}$ and $\mathcal{I}^{\prime \prime \prime}$ ) does not affect $Q_{1}\left(\mathcal{I}^{\prime \prime}\right)$. Similarly, adding $(0)$ to $I_{2}^{\prime \prime}$ (as done in the extensions $\mathcal{I}$ and $\mathcal{I}^{\prime}$ ) does not affect $Q_{2}\left(\mathcal{I}^{\prime \prime}\right)$ since $\varphi_{2}$ is true. Hence, 
$Q\left(\mathcal{I}^{\prime \prime}\right)=Q(\mathcal{I})=Q\left(\mathcal{I}^{\prime}\right)=Q\left(\mathcal{I}^{\prime \prime \prime}\right)$ and $\mathcal{I}^{\prime \prime}$ is indeed in $\operatorname{RCQ}^{s}\left(Q, D_{m}, V\right)$; this shows that $\mathcal{I}$ is not minimal.

Upper bound. We show that, for ground instances, $\operatorname{MINP}\left(\exists \mathrm{FO}^{+}\right)$is in $\mathrm{D}_{2}^{p}$. By Lemma 4.7, the set of yes-instances to $\operatorname{MINP}\left(\exists \mathrm{FO}^{+}\right)$is $L_{1} \cap L_{2}$, where

$-L_{1}=\left\{\left(I, Q, D_{m}, V\right) \mid I \in \mathrm{RCQ}^{s}\left(Q, D_{m}, V\right)\right\} ;$ and

$-L_{2}=\left\{\left(I, Q, D_{m}, V\right) \mid\right.$ for all $\left.t \in I, I \backslash\{t\} \notin \operatorname{RCQ}\left(Q, D_{m}, V\right)\right\}$.

It now suffices to show that $L_{1} \in \Pi_{2}^{p}$ and $L_{2} \in \Sigma_{2}^{p}$. Clearly, $L_{1} \in \Pi_{2}^{p}$ follows from Theorem 4.1. To show that $L_{2} \in \Sigma_{2}^{p}$, we modify the algorithm for the complement problem of $\operatorname{RCDP}\left(\exists \mathrm{FO}^{+}\right)$given in the proof of Theorem 4.1. More specifically, consider $\left(I, Q, D_{m}, V\right)$ and assume that $I=\left\{t_{1}, \ldots, t_{k}\right\}$. Let $I_{i}=I \backslash\left\{t_{i}\right\}$ for $i \in[1, k]$. We then apply the $\Sigma_{2}^{p}$-algorithm for each $\left(I_{i}, Q, D_{m}, V\right)$ "in parallel," that is, the algorithm guesses $k$ tuples $s_{i}$ (by means of a valuation of the query $Q$ ) such that $s_{i} \in Q\left(I_{i}^{\prime}\right) \backslash Q\left(I_{i}\right)$ for some partially closed extension $I_{i}^{\prime}$ of $I_{i}$ (also identified by the valuation of the query $Q$ ). We can make such $k$ guesses at the same time since there are only polynomially many $(k=|I|)$ instances $I_{i}$. The algorithm rejects a guess as long as any of the guessed $s_{i} \in Q\left(I_{i}\right)$ or $I_{i}^{\prime}$ is not partially closed for some $i \in[1, k]$. However, when guesses are accepted, we have found $k$ witnesses showing that none of the $I_{i}$ s are in $\operatorname{RCQ}^{s}\left(Q, D_{m}, V\right)$. In other words, $\left(I, Q, D_{m}, V\right) \in L_{2}$.

\section{WEAK RELATIVE INFORMATION COMPLETENESS}

We next investigate RCDP, RCQP, and MINP for weakly complete databases, denoted by $\mathrm{RCDP}^{w}, \mathrm{RCQP}^{w}$, and MINP ${ }^{w}$, respectively. We consider the databases from which one can find the certain answers to a query over their partially closed extensions. Here, we denote by $\operatorname{RCQ}^{w}\left(Q, D_{m}, V\right)$ the set of instances that are weakly complete. In the weak completeness model, none of these problems has been studied before, neither for $c$-instances nor ground instances. We provide their complexity bounds here.

Compared to their counterparts in the strong model, the complexity results in the weak model are more diverse. On the one hand, the certain-answer semantics simplifies the analysis of some problems; for example, all these problems become decidable for FP, in contrast to their undecidability in the strong model. On the other hand, it makes certain problems harder; for example, $\mathrm{MINP}^{w}$ becomes $\Pi_{4}^{p}$-complete for $\mathrm{UCQ}$, as opposed to $\Pi_{3}^{p}$ for MINP ${ }^{s}$. In addition, some problems even have different bounds for $\mathrm{CQ}$ and $\mathrm{UCQ}$; for example, MINP ${ }^{w}$ is coDP-complete for $\mathrm{CQ}$, while it is $\Pi_{4}^{p}$-complete for UCQ.

\subsection{The Relatively Complete Database Problem in the Weak Model}

As opposed to Theorem 4.1, RCDP ${ }^{w}$ is decidable for FP. In addition, RCDP ${ }^{w}$ for $c$ instances and $\operatorname{RCDP}^{w}$ for ground instances are both $\Pi_{3}^{p}$-complete when $\mathcal{L}_{Q}$ is $\mathrm{CQ}, \mathrm{UCQ}$ or $\exists \mathrm{FO}^{+}$, while their counterparts in the strong model are $\Pi_{2}^{p}$-complete (Theorem 4.1).

Theorem 5.1. For c-instances and for ground instances, $\operatorname{RCDP}^{w}\left(\mathcal{L}_{Q}\right)$ is

-undecidable when $\mathcal{L}_{Q}$ is $\mathrm{FO}$,

- coneXPTIME-complete when $\mathcal{L}_{Q}$ is FP, and

- $\Pi_{3}^{p}$-complete when $\mathcal{L}_{Q}$ is $C Q, U C Q$ or $\exists F O^{+}$.

Proof. We show that, in the weak model, $\operatorname{RCDP}^{w}\left(\mathcal{L}_{Q}\right)$ is undecidable when $\mathcal{L}_{Q}$ is $\mathrm{FO}$, conEXPTIME-complete when $\mathcal{L}_{Q}$ is FP, and it becomes $\Pi_{3}^{p}$-complete when $\mathcal{L}_{Q}$ is $\mathrm{CQ}$, UCQ, or $\exists \mathrm{FO}^{+}$. The lower bounds hold even when only ground instances are considered, and when $D_{m}$ and $V$ are fixed. 
(1) When $\mathcal{L}_{Q}$ is FO. To prove the undecidability, it suffices to consider ground instances without variables only. Indeed, a ground instance is also a $c$-instance.

We prove the undecidability by reduction from a variant of the satisfiability problem for FO. It is to decide, given an FO query $q$ such that $q(\emptyset)=\emptyset$ (i.e., $q$ is not satisfied by the empty instance), whether $q$ is satisfiable. It is easy to verify that this variant of FO satisfiability is also undecidable by reduction from $\mathrm{FO}$ satisfiability.

Consider an FO query $q$ such that $q(\emptyset)=\emptyset$. Assume without loss of generality by Lemma 3.2 that the given FO query $q$ is defined on a single relation $R$. Then, we define $Q$ over $R$ such that, for every instance $I$ of $R, Q(I)=\{()\}$ if $q(I)=\emptyset$, and $Q(I)=\varnothing$ otherwise. We define $D_{m}$ to be an empty instance, and $V$ to be the empty set. We show that $q$ is not satisfiable if and only if the empty instance $\emptyset$ is in $\operatorname{RCQ}^{w}\left(Q, D_{m}, V\right)$.

$\Rightarrow$ First, assume that $q$ is not satisfiable, that is, for all instances $I$ of $R, q(I)=\emptyset$. Then, for all $I \in \operatorname{Ext}(\emptyset), Q(I)=\{()\}$; hence, $\bigcap_{I \in \operatorname{Ext}(\emptyset)} Q(I)=\{()\}=Q(\varnothing)$. Thus, $\emptyset$ is in $\operatorname{RCQ}\left(Q, D_{m}, V\right)$.

$\Leftarrow$ Conversely, assume that $q$ is satisfiable, that is, there exists an instance $I_{0}$ of $R$ such that $q\left(I_{0}\right)$ is not empty. Then, by the definition of $Q, Q\left(I_{0}\right)=\emptyset=\bigcap_{I \in \operatorname{Ext}(\emptyset)} Q(I) \neq$ $Q(\emptyset)=\{()\}$, since $q(\emptyset)=\emptyset$. Hence, $\varnothing$ is not in $\operatorname{RCQ}\left(Q, D_{m}, V\right)$.

We remark that, in this proof, the master data $D_{m}$ and the set $V$ of CCs are fixed, independent of the input query $q$. In fact, they are even absent.

(2) When $\mathcal{L}_{Q}$ is FP. We show that $\operatorname{RCDP}^{w}(\mathrm{FP})$ is conexptime-complete. That is, we show that $\mathrm{RCDP}^{w}(\mathrm{FP})$ is already conEXPTIME-hard for ground instances, and is in conEXPTIME for arbitrary $c$-instances.

Lower bound. We show that, for ground instances, RCDP ${ }^{w}(\mathrm{FP})$ is coneXPTIME-hard by reduction from the SUCCINCT-TAUT problem, which is coNEXPTIME-complete (see Papadimitriou [1994]). An instance of SUCCINCT-TAUT is defined by a Boolean circuit $C$ consisting of a finite set of gates $\left\{g_{i}=\left(a_{i}, j, k\right) \mid 1 \leq i \leq M\right\}$, where $a_{i} \in\{\wedge, \vee, \neg$, in $\}$ is the type of the gate $g_{i}, g_{j}$ and $g_{k}$ for $j, k<i$ are the inputs of the gate (unless $g_{i}$ is an in-gate, in which case $j=k=0$, or unless $g_{i}$ is a $\neg$-gate, in which case $j=k$ ). Suppose that $C$ has $n$ input gates; then, $C$ defines the Boolean function $f_{C}:\{0,1\}^{n} \rightarrow\{0,1\}$, where $f_{C}(\bar{w})=1$ if and only if $C$ evaluates to true on input $\bar{w}$. The succinct-TAUT problem is to decide whether for all $\bar{w} \in\{0,1\}^{n}, f_{C}(\bar{w})=1$, that is, whether $C$ is a tautology.

Given an instance of the latter problem, we define database schemas $\mathcal{R}$ and $\mathcal{R}_{m}$, a ground instance $\mathcal{I}$ of $\mathcal{R}$, a set $V$ of CCs, master data $D_{m}$ of $\mathcal{R}_{m}$, and an FP query $Q$. We show that $C$ is a tautology if and only if $\mathcal{I}$ is a minimal instance in $\operatorname{RCQ}^{w}\left(Q, D_{m}, V\right)$.

(a) The database schema $\mathcal{R}$ consists of a single relation $R\left(A_{0}, A_{1}, \ldots, A_{30}\right)$, where, for a tuple $t$ of $R, t\left[A_{1}, A_{2}\right]$ is to encode $I_{(0,1)} ; t\left[A_{3}, \ldots, A_{14}\right]$ encodes $I_{\vee} ; t\left[A_{15}, \ldots, A_{26}\right]$ encodes $I_{\wedge}$, and $t\left[A_{27}, \ldots, A_{30}\right]$ encodes $I_{\neg}$.

(b) A ground instance $\mathcal{I}$ of $\mathcal{R}$ consists of a single tuple $t$ that is formed by juxtaposing instances $I_{(0,1)}, I_{\vee}, I_{\wedge}$, and $I_{\neg}$ in $t\left[A_{1}, \ldots, A_{30}\right]$, with $t\left[A_{0}\right]=1$. Here, $I_{(0,1)}, I_{\vee}, I_{\wedge}$, and $I_{\neg}$ are given in Figure 2.

(c) The master data $D_{m}$ and CCs in $V$ ensure that every tuple $t$ of $R$ satisfies the following: (i) $t\left[A_{1}, \ldots, A_{30}\right]$ encodes the instances mentioned earlier; and (ii) $t\left[A_{0}\right]$ only takes values from $\{0,1\}$.

(d) The query $Q$ in FP uses an $(n+1)$-ary IDB predicates $G_{i}$, one for each gate $g_{i}=\left(a_{i}, j, k\right)$ in $C$, a unary IDB $I$ to encode $I_{(0,1)}$, and an $n$-ary IDB $R_{X}$ to encode all possible $n$-ary binary 
tuples. That is, we include the following FP rules in $Q$ :

$$
\begin{aligned}
I(x) & \leftarrow R\left(A_{0}, x, A_{2}, \ldots, A_{30}\right) ; \\
I(x) & \leftarrow R\left(A_{0}, A_{1}, x, A_{3}, \ldots, A_{30}\right) ; \\
R_{X}(\vec{x}) & \leftarrow I\left(x_{1}\right), \ldots, I\left(x_{n}\right) ; \\
\text { If } a_{i}=\text { in, then } G_{i}(B, \vec{x}) & \leftarrow R_{X}(\vec{x}), B=x_{i} ; \\
\text { If } a_{i}=\vee \text {, then } G_{i}(B, \vec{x}) & \leftarrow G_{j}\left(B_{1}, \vec{x}\right), G_{k}\left(B_{2}, \vec{x}\right), R\left(A_{0}, A_{1}, A_{2}, B_{1}, B_{2}, B, A_{6}, \ldots, A_{30}\right) ; \\
\vdots & \vdots \\
\text { If } a_{i}=\vee \text {, then } G_{i}(B, \vec{x}) & \leftarrow G_{j}\left(B_{1}, \vec{x}\right), G_{k}\left(B_{2}, \vec{x}\right), R\left(A_{0}, \ldots, A_{11}, B_{1}, B_{2}, B, A_{15}, \ldots, A_{30}\right) ; \\
\text { If } a_{i}=\wedge \text {, then } G_{i}(B, \vec{x}) & \leftarrow G_{j}\left(B_{1}, \vec{x}\right), G_{k}\left(B_{2}, \vec{x}\right), R\left(A_{0}, \ldots, A_{14}, B_{1}, B_{2}, B, A_{18}, \ldots, A_{30}\right) ; \\
\vdots & \vdots \\
\text { If } a_{i}=\wedge \text {, then } G_{i}(B, \vec{x}) & \leftarrow G_{j}\left(B_{1}, \vec{x}\right), G_{k}\left(B_{2}, \vec{x}\right), R\left(A_{0}, \ldots, A_{23}, B_{1}, B_{2}, B, A_{27}, \ldots, A_{30}\right) ; \\
\text { If } a_{i}=\neg \text {, then } G_{i}(B, \vec{x}) & \leftarrow G_{j}\left(B_{1}, \vec{x}\right), R\left(A_{0}, \ldots, A_{26}, B_{1}, B, A_{29}, A_{30}\right) ; \\
\text { If } a_{i}=\neg \text {, then } G_{i}(B, \vec{x}) & \leftarrow G_{j}\left(B_{1}, \vec{x}\right), R\left(A_{0}, \ldots, A_{28}, B_{1}, B\right) ;
\end{aligned}
$$

and, finally, two more rules:

$$
\begin{aligned}
& G(\vec{x}) \leftarrow G_{M}(B, \vec{x}), R\left(0, A_{1}, \ldots, A_{30}\right), \\
& G(\vec{x}) \leftarrow G_{M}(B, \vec{x}), B=1,
\end{aligned}
$$

where $G_{M}$ is the IDB corresponding to the output gate $g_{M}$. Intuitively, $Q(\mathcal{I})$ will return all $\bar{w} \in\{0,1\}^{n}$ for which $f_{C}(\bar{w})=1$.

We show that $C$ is a tautology if and only if $\mathcal{I} \in \mathrm{RCQ}^{w}\left(Q, D_{m}, V\right)$.

$\Rightarrow$ First, assume that $C$ is a tautology. We show that $\mathcal{I}$ is weakly complete for $Q$ relative to $\left(D_{m}, V\right)$. Since for all $\bar{w} \in\{0,1\}^{n}, f_{C}(\bar{w})=1, Q(\mathcal{I})$ will return all $\bar{w} \in\{0,1\}^{n}$. Observe that the only extension $\mathcal{I}^{\prime}$ of $\mathcal{I}$ is $\left\{t, t^{\prime}\right\}$, where $t$ is in $\mathcal{I}$ and $t^{\prime}$ is the same as $t$ except that $t^{\prime}\left[A_{0}\right]=0$ while $t\left[A_{0}\right]=1$. Obviously, we have that $Q\left(\mathcal{I}^{\prime}\right)$ returns all $\bar{w} \in\{0,1\}^{n}$ as well. Hence, $\mathcal{I}$ is weakly complete.

$\Leftarrow$ Conversely, suppose that $C$ is not a tautology but $\mathcal{I}$ is weakly complete. Note again that the ground instance $\mathcal{I}^{\prime}$ mentioned earlier is the only extension of $\mathcal{I}$ and, furthermore, that $Q\left(\mathcal{I}^{\prime}\right)$ contains all $\bar{w} \in\{0,1\}^{n}$. Hence, in order for $\mathcal{I}$ to be weakly complete, $Q(\mathcal{I})$ must contain all $\bar{w} \in\{0,1\}^{n}$. This, however, contradicts the assumption that $C$ is not a tautology since $Q(\mathcal{I})$ contains only those $\bar{w} \in\{0,1\}^{n}$ for which $f_{C}(\bar{w})=1$ (recall that $t\left[A_{0}\right]=1$ ). Hence, $\mathcal{I}$ cannot be weakly complete.

Upper bound. We show that $\operatorname{RCDP}^{w}(\mathrm{FP})$ is in conEXPTIME by providing an NEXPTIME algorithm that decides the complement problem. That is, given a $c$-instance $\mathcal{T}$, master data $D_{m}$, a set $V$ of CCs, and an FP query $Q$, the algorithm returns "yes" if $\mathcal{T}$ is not weakly complete for $Q$ relative to $\left(D_{m}, V\right)$, and "no" otherwise.

To do this, we first give a sufficient and necessary condition for characterizing weak completeness by the following lemma. By Lemma 3.2, we assume without loss of generality that $\mathcal{R}$ consists of a single relation schema $R$ and $\mathcal{T}$ is a c-table $(T, \xi)$. Recall the notion of Adom given in the proof of Theorem 4.1.

Lemma 5.2. For every $Q$ in FP, master data $D_{m}$, set $V$ of $C C$, and any c-instance $\mathcal{T}=(T, \xi)$, let $\mathcal{T}^{\prime}=\left(T \cup\left\{\left(x_{1}, \ldots, x_{n}\right), \xi\right\}\right)$, that is, $\mathcal{T}$ extended with a single tuple consisting of (new) variables only. Then, $(T, \xi)$ is not in $\operatorname{RCQ}^{w}\left(Q, D_{m}, V\right)$ if and only if 
there exist a tuple $t$ and an instance $\mathcal{I} \in \operatorname{Mod}_{\text {Adom }}(\mathcal{T})$ such that $t \in \bigcap_{\mathcal{I}^{\prime} \in \operatorname{Mod} \operatorname{Adom}\left(\mathcal{I}^{\prime}\right)} Q\left(\mathcal{I}^{\prime}\right)$ and $t \notin Q(\mathcal{I})$.

Proof. First, assume that $(T, \xi)$ is not in $\operatorname{RCQ}^{w}\left(Q, D_{m}, V\right)$. Then, $\bigcap_{\mathcal{I} \in \operatorname{Mod}(\mathcal{T})} Q(\mathcal{I}) \neq$ $\bigcap_{\mathcal{I} \in \operatorname{Mod}(\mathcal{T}), \mathcal{I}^{\prime} \in \operatorname{Ext}(\mathcal{I})} Q\left(\mathcal{I}^{\prime}\right)$. By the monotonicity of FP, we have that $\bigcap_{\mathcal{I} \in \operatorname{Mod}(\mathcal{T})} Q(\mathcal{I}) \subseteq$ $\bigcap_{\mathcal{I} \in \operatorname{Mod}(\mathcal{T}), \mathcal{I}^{\prime} \in \operatorname{Ext}(\mathcal{I})} Q\left(\mathcal{I}^{\prime}\right)$. Thus, there must exist a tuple $t^{\prime}=\left(a_{1}, \ldots, a_{n}\right)$ such that $t^{\prime} \in \bigcap_{\mathcal{I} \in \operatorname{Mod}(\mathcal{T}), \mathcal{I}^{\prime} \in \operatorname{Ext}(\mathcal{I})} Q\left(\mathcal{I}^{\prime}\right)$ and $t^{\prime} \notin \bigcap_{\mathcal{I} \in \operatorname{Mod}(\mathcal{T})} Q(\mathcal{I})$. In other words, there exists an $\mathcal{I} \in \operatorname{Mod}(\mathcal{T})$ such that $t^{\prime} \in \bigcap_{\mathcal{I} \in \operatorname{Mod}(\mathcal{T}), \mathcal{I}^{\prime} \in \operatorname{Ext}(\mathcal{I})} Q\left(\mathcal{I}^{\prime}\right)$ and $t^{\prime} \notin Q(\mathcal{I})$. Note that $\bigcap_{\mathcal{I} \in \operatorname{Mod}(\mathcal{T}), \mathcal{I}^{\prime} \in \operatorname{Ext}(\mathcal{I})} Q\left(\mathcal{I}^{\prime}\right) \subseteq \bigcap_{\mathcal{I}^{\prime} \in \operatorname{Mod}\left(\mathcal{T}^{\prime}\right)} Q\left(\mathcal{I}^{\prime}\right)$, and then $t^{\prime} \in \bigcap_{\mathcal{I}^{\prime} \in \operatorname{Mod}\left(\mathcal{T}^{\prime}\right)} Q\left(\mathcal{I}^{\prime}\right)$. Construct a tuple $t=\left(b_{1}, \ldots, b_{n}\right)$ from $t^{\prime}$ that takes values from Adom, such that, for each $i \in[1, n]$, $b_{i}=a_{i}$ if $a_{i}$ is a constant appearing in $\mathcal{T}, D_{m}, V$, or $Q$; otherwise, $b_{i}$ takes values from new constants in Adom defined early. One can readily verify that $t$ is in $\bigcap_{\mathcal{I}^{\prime} \in \operatorname{Mod}\left(\mathcal{I}^{\prime}\right)} Q\left(\mathcal{I}^{\prime}\right)$

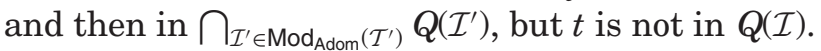

Conversely, suppose that there exist a tuple $t$ and an instance $\mathcal{I} \in \operatorname{Mod}_{\text {Adom }}(\mathcal{T})$ such that $t \in \bigcap_{\mathcal{I}^{\prime} \in \operatorname{Mod}_{\text {Adom }}\left(\mathcal{T}^{\prime}\right)} Q\left(\mathcal{I}^{\prime}\right)$ and $t \notin Q(\mathcal{I})$. Observe that $t \in \bigcap_{\mathcal{I} \in \operatorname{Mod}(\mathcal{T}), \mathcal{I}^{\prime} \in \operatorname{Ext}(\mathcal{I})} Q\left(\mathcal{I}^{\prime}\right)$. Indeed, suppose otherwise. Then there exist $\mathcal{I}_{1} \in \operatorname{Mod}(\mathcal{T})$ and $\mathcal{I}_{2} \in \operatorname{Ext}(\mathcal{I})$ such that $t \notin Q\left(\mathcal{I}_{2}\right)$. By the monotonicity of FP, $t \notin Q\left(\mathcal{I}_{3}\right)$ for every $\mathcal{I}_{3}=\mathcal{I}_{1} \cup\{s\}$ with $s \in \mathcal{I}_{2} \backslash \mathcal{I}_{1}$. Pick such an $\mathcal{I}_{3}$ and let $v^{\prime}$ be the corresponding valuation of $\mathcal{T}^{\prime}$ taking values from $\mathcal{I}_{3}$. This induces a valuation $\mu^{\prime}$ of $\mathcal{T}^{\prime}$ with values in Adom such that $t \notin Q\left(\mu^{\prime}\left(\mathcal{T}^{\prime}\right)\right)$, contradicting the assumption that $t \in \bigcap_{\mathcal{I}^{\prime} \in \operatorname{Mod}_{\text {Adom }}\left(\mathcal{T}^{\prime}\right)} Q\left(\mathcal{I}^{\prime}\right)$. Thus, $\mathcal{T}$ is not weakly complete.

Capitalizing on the characterization, we next present the NEXPTIME algorithm.

(1) Guess a tuple $t$ of the (output) schema of $Q$ with values from Adom.

(2) Check whether $t \notin Q(\mu(\mathcal{T}))$ for some valuation $\mu$ of $\mathcal{T}$ taking values from Adom; if so, continue; otherwise, reject the guess. Since the valuations range over a finite domain, each $\mu(\mathcal{T})$ is of polynomial size, and evaluating FP queries takes EXPTIME, the total cost of this process is EXPTIME.

(3) For $\mathcal{T}^{\prime}=\mathcal{T} \cup\left\{\left(x_{1}, \ldots, x_{n}\right)\right\}$, we test whether $t \in Q\left(\mu^{\prime}\left(\mathcal{T}^{\prime}\right)\right)$ for each valuation $\mu^{\prime}$ of $\mathcal{T}^{\prime}$ such that $\left(\mu^{\prime}\left(\mathcal{T}^{\prime}\right), D_{m}\right) \models V,\left(\mu^{\prime}(\mathcal{T}), D_{m}\right) \models V$ and $\mu^{\prime}(\mathcal{T}) \subsetneq \mu^{\prime}\left(\mathcal{T}^{\prime}\right)$. If successful, the algorithm returns "yes." Otherwise, the current guess is rejected. For the same reason as outlined earlier, this process takes EXPTIME.

The overall complexity of the algorithm is NEXPTIME; hence, $\operatorname{RCDP}^{w}(\mathrm{FP})$ is in conEXPTIME. Obviously, the algorithm is correct, by Lemma 5.2. The algorithm returns "yes" if and only if there exists a tuple $t$ and instance $\mathcal{I} \in \operatorname{Mod}_{\text {Adom }}(\mathcal{T})$ such that $t \in$ $\bigcap_{\mathcal{I}^{\prime} \in \operatorname{Mod}_{A d o m}\left(\mathcal{T}^{\prime}\right)} Q\left(\mathcal{I}^{\prime}\right)$ and $t \notin Q(\mathcal{I})$.

(3) When $\mathcal{L}_{Q}$ is $\mathrm{CQ}, \mathrm{UCQ}$, or $\exists \mathrm{FO}^{+}$. It suffices to show that $\mathrm{RCDP}^{w}(\mathrm{CQ})$ is $\Pi_{3}^{p}$-hard for ground instances and $\operatorname{RCDP}^{w}\left(\exists \mathrm{FO}^{+}\right)$is in $\Pi_{3}^{p}$ for $c$-instances.

Lower bound. We show that, for ground instances, $\operatorname{RCDP}^{w}(\mathrm{CQ})$ is $\Pi_{3}^{p}$-hard by reduction from the complement of the $\exists^{*} \forall^{*} \exists^{*}$ 3saT-problem. Given a formula $\varphi=\exists X \forall Y \exists Z \psi$, $\exists^{*} \forall^{*} \exists^{*} 3 \mathrm{SAT}$ is to determine whether $\varphi$ is true. Here, $X=\left\{x_{1}, \ldots, x_{n}\right\}, Y=\left\{y_{1}, \ldots, y_{m}\right\}$, and $Z=\left\{z_{1}, \ldots, z_{l}\right\}$, which are sets of variables; and $\psi=C_{1} \wedge \cdots \wedge C_{r}$ is an instance of 3sat. It is known that $\exists^{*} \forall^{*} \exists * 3$ SAT is $\Sigma_{3}^{p}$-complete (see Papadimitriou [1994]).

Given an instance $\varphi=\exists X \forall Y \exists Z \psi$ of $\exists^{*} \forall^{*} \exists * 3$ sAT, we define database schemas $\mathcal{R}$ and $\mathcal{R}_{m}$, a ground instance $\mathcal{I}$, a set $V$ of CCs, master data $D_{m}$, and a CQ query $Q$. We show that $\varphi$ is true if and only if $\mathcal{I}$ is not weakly complete for $Q$ relative to $\left(D_{m}, V\right)$.

(a) The database schema $\mathcal{R}$ consists of five relation schemas: $R_{(0,1)}(A), R_{\neg}(A, \bar{A})$, $R_{\vee}\left(A_{1}, A_{2}, B\right)$, and $R_{\wedge}\left(A_{1}, A_{2}, B\right)$, which are the same as their counterparts given in 
the proof of Proposition 3.3, respectively. In addition, $\mathcal{R}$ contains one extra relation $R_{Y}\left(Y_{1}, \ldots, Y_{m}\right)$ to generate truth assignments of $Y$ variables.

(b) The ground instance $\mathcal{I}$ is given by $\left(I_{(0,1)}, I_{\neg}, I_{\vee}, I_{\wedge}, I_{Y}\right)$. Here, $I_{(0,1)}, I_{\neg}, I_{\vee}$, and $I_{\wedge}$ are the instances shown in Figure 2, and $I_{Y}$ is an empty instance of $R_{Y}$.

(c) The schema $\mathcal{R}_{m}$ of master data contains $R_{(0,1)}^{m}=R_{(0,1)}, R_{\neg}^{m}=R_{\neg}, R_{\vee}^{m}=R_{\vee}, R_{\wedge}^{m}=R_{\wedge}$, and $R_{\emptyset}\left(W, W^{\prime}\right)$. The master data instance $D_{m}$ consists of $I_{(0,1)}^{m}=I_{(0,1)}, I_{\neg}^{m}=I_{\neg}, I_{\vee}^{m}=I_{\vee}$, $I_{\wedge}^{m}=I_{\wedge}$, and $I_{\emptyset}^{m}=\emptyset$.

(d) The set $V$ of CCs consists of the following CCs:

$-R_{(0,1)} \subseteq R_{(0,1)}^{m}, R_{\vee} \subseteq R_{\vee}^{m}, R_{\wedge} \subseteq R_{\wedge}^{m}, R_{\neg} \subseteq R_{\neg}^{m} ;$

一 $\phi_{i}: q_{i}\left(y_{i}\right) \subseteq R_{(0,1)}$, where $q_{i}\left(y_{i}\right)=\exists y_{1} \ldots y_{i-1} y_{i+1} \ldots y_{m}\left(R_{Y}\left(y_{1}, \ldots, y_{m}\right)\right), i \in[1, m]$; and

$-\phi_{i}^{\prime}: q_{i}^{\prime}\left(y_{i}, y_{i}^{\prime}\right) \subseteq R_{\emptyset}$, where, for $i \in[1, m], q_{i}^{\prime}\left(y_{i}, y_{i}^{\prime}\right)=\exists y_{1} y_{1}^{\prime} \ldots y_{i-1} y_{i-1}^{\prime} y_{i+1} y_{i+1}^{\prime} \ldots y_{m} y_{m}^{\prime}$ $\left(R_{Y}\left(y_{1}, \ldots, y_{m}\right) \wedge R_{Y}\left(y_{1}^{\prime}, \ldots, y_{m}^{\prime}\right) \wedge y_{i} \neq y_{i}^{\prime}\right)$.

It is easy to see that, for each extension $I_{Y}^{\prime}$ of $I_{Y}, I_{Y}^{\prime} \models \bigwedge_{i \in[1, m]}\left(\phi_{i} \wedge \phi_{i}^{\prime}\right)$ if and only if $I_{Y}^{\prime}$ consists of a single tuple that encodes a valid truth assignment of $Y$.

(e) We define the CQ query $Q$ as follows:

$$
Q(\vec{x})=\exists \vec{y}, \vec{z} Q_{X}(\vec{x}) \wedge R_{Y}(\vec{y}) \wedge Q_{Z}(\vec{z}) \wedge Q_{\psi}(\vec{x}, \vec{y}, \vec{z}, w) \wedge w=1,
$$

where $\vec{x}=\left(x_{1}, \ldots, x_{n}\right), \vec{y}=\left(y_{1}, \ldots, y_{m}\right)$, and $\vec{z}=\left(z_{1}, \ldots, z_{l}\right)$. The subqueries $Q_{X}(\vec{x})=$ $\bigwedge_{i=1}^{m} R_{01}\left(x_{i}\right)$ and $Q_{Z}(\vec{z})=\bigwedge_{i=1}^{l} R_{01}\left(z_{i}\right)$ generate all valid truth assignments of $X$ variables and $Z$ variables, respectively, by means of Cartesian products of $R_{01}$. Given truth assignments $\left(\mu_{X}, \mu_{Y}, \mu_{Z}\right)$ of $(X, Y, Z)$, query $Q_{\psi}$ is to encode the truth value of $\psi\left(\mu_{X}, \mu_{Y}, \mu_{Z}\right)$ as the value of $w$. Obviously, $Q_{\psi}$ can be expressed in CQ in terms of $R_{\vee}$, $R_{\wedge}$, and $R_{\neg}$. Intuitively, query $Q$ returns all truth assignments $\mu_{X}$ of $X$ if there exists truth assignments $\mu_{Y}$ and $\mu_{Z}$ of $Y$ and $Z$, respectively, that make $\psi$ true.

We next show that the reduction is correct, that is, $\varphi$ is true if and only if $\mathcal{I}$ is not weakly complete for $Q$ relative to $\left(D_{m}, V\right)$.

$\Rightarrow$ First, assume that $\varphi$ is true. Then, there exists a truth assignment $\mu_{X}^{0}$ of $X$ such that, for each truth assignment $\mu_{Y}$ of $Y$, there exists a truth assignment $\mu_{Z}$ of $Z$ such that $\psi\left(\mu_{X}, \mu_{Y}, \mu_{Z}\right)$ is true. We next show that $\mathcal{I}$ is not weakly complete for $Q$ relative to $\left(D_{m}, V\right)$. That is, we need to show that $Q(\mathcal{I}) \neq \bigcap_{\mathcal{I}^{\prime} \in \operatorname{Ext}(\mathcal{I})} Q\left(\mathcal{I}^{\prime}\right)$. Observe the following: (i) $Q(\mathcal{I})=\emptyset$ since $I_{Y}$ is empty; and (ii) for each instance $\mathcal{I}^{\prime}$ in $\operatorname{Ext}(\mathcal{I}), \mathcal{I}^{\prime}=$ $\left(I_{(0,1)}, I_{\neg}, I_{\vee}, I_{\wedge}, I_{Y}^{\prime}\right)$, where $I_{Y}^{\prime}$ encodes a valid truth assignment of $Y$, and $\left(\vec{x}_{0}\right) \in Q\left(\mathcal{I}^{\prime}\right)$ represents the truth assignment $\mu_{X}^{0}$. Thus, $\left(\vec{x}_{0}\right) \in \bigcap_{\mathcal{I}^{\prime} \in \operatorname{Ext}(\mathcal{I})} Q\left(\mathcal{I}^{\prime}\right)$. Hence, $\mathcal{I}$ is not weakly complete for $Q$ relative to $\left(D_{m}, V\right)$.

$\Leftarrow$ Conversely, if $\varphi$ is false, then there exists no truth assignment $\mu_{X}$ of $X$ such that, for all truth assignments of $Y$, there exists a truth assignment of $Z$ that makes $\psi$ true. Recall that, for each partially closed extension $\mathcal{I}^{\prime}$ of $\mathcal{I}, \mathcal{I}^{\prime}$ can only be the form of $\left(I_{(01)}, I_{\neg}, I_{\vee}, I_{\wedge}, I_{Y}^{\prime}\right)$, where $I_{Y}^{\prime}$ encodes a truth assignment of $Y$. By the definition of $Q$, if there exists a tuple $t$ in $\bigcap_{\mathcal{I}^{\prime} \in \operatorname{Ext}(\mathcal{I})} Q\left(\mathcal{I}^{\prime}\right), t$ must encode a truth assignment of $X$ such that, for every truth assignment of $Y$, there exists a truth assignment of $Z$ that makes $\psi$ true. Thus, $\varphi$ is true, which contradicts the assumption that $\varphi$ is false. As a result, $\bigcap_{\mathcal{I}^{\prime} \in \operatorname{Ext}(\mathcal{I})} Q\left(\mathcal{I}^{\prime}\right)$ must be empty, and $\mathcal{I}$ is weakly complete for $Q$ relative to $\left(D_{m}, V\right)$.

Upper bound. It suffices to show that $\operatorname{RCDP}^{w}\left(\exists \mathrm{FO}^{+}\right)$is in $\Pi_{3}^{p}$. To do this, we use the same algorithm given for $\operatorname{RCDP}^{w}(\mathrm{FP})$. We show the algorithm is in $\Sigma_{3}^{p}$; then, $\operatorname{RCDP}^{w}\left(\exists \mathrm{FO}^{+}\right)$ is in $\Pi_{3}^{p}$. Step (2) of the algorithm can be done in $\Sigma_{2}^{p}$ by the following procedure. 
(1) Guess a valuation $\mu$ of $\mathcal{T}$ by taking values from Adom.

(2) Check whether $\mu(\mathcal{T})$ satisfies $\xi$. If so continue; otherwise, reject the guess.

(3) Check whether $t \notin Q(\mu(\mathcal{T}))$. If so, return "yes"; otherwise reject the guess.

Obviously, it returns "yes" if and only if there exists a valuation of $\mathcal{T}$ such that $t \notin$ $Q(\mu(\mathcal{T}))$. It is in $\Sigma_{2}^{p}$ since Step (2) is in PTIME and Step (3) is in coNP for CQ.

Moreover, Step (3) of the algorithm is also in $\Sigma_{2}^{p}$ for $\exists \mathrm{FO}^{+}$. We give a $\Pi_{2}^{p}$ procedure for the complement of Step (3), that is, it returns "no" if and only if $t \notin \bigcap_{I^{\prime} \in \operatorname{Mod}_{\text {Adom }}\left(\mathcal{T}^{\prime}\right)} Q\left(I^{\prime}\right)$.

(1) Guess a valuation $\mu^{\prime}$ of $\left(\mathcal{T}^{\prime}, \xi^{\prime}\right)$ by taking values from Adom.

(2) Check whether $\mu^{\prime}\left(\mathcal{T}^{\prime}\right)$ satisfies $\xi^{\prime}$. If so, continue; otherwise, reject the guess.

(3) Check whether $\left(\mu^{\prime}\left(\mathcal{T}^{\prime}\right), D_{m}\right) \models V$ and whether $\left(\mu^{\prime}(\mathcal{T}), D_{m}\right) \models V$. If so, continue; otherwise, reject the guess.

(4) Check whether $\mu^{\prime}(\mathcal{T}) \varsubsetneqq \mu^{\prime}\left(\mathcal{T}^{\prime}\right)$. If so, continue; otherwise, reject the guess.

(5) Check if $t \notin Q\left(\mu^{\prime}\left(T^{\prime}\right)\right)$. If so, return "no"; otherwise, reject the guess.

It returns "no" if and only if $t \notin \bigcap_{I^{\prime} \in \operatorname{Mod}} \operatorname{Mdom}_{\left(\mathcal{T}^{\prime}\right)} Q\left(I^{\prime}\right)$. It is in $\Pi_{2}^{p}$ since Step (2) is in PTIME, Step (3) is in NP, and Steps (4) and (5) are both in coNP, for $\exists \mathrm{FO}^{+}$queries.

\subsection{The Relatively Complete Query Problem in the Weak Model}

Recall that $\mathrm{RCQP}^{s}$ for $c$-instances is equivalent to $\mathrm{RCQP}^{s}$ for ground instances, as verified by Lemma 4.4. However, the following example tells us that it is no longer the case in the weak completeness model.

Example 5.3. Consider an FO query $Q$ defined on a pair of relations: $Q\left(I_{1}, I_{2}\right)=\{(a)\}$ if $I_{1} \subseteq I_{2}$, and it is $\{(b)\}$ otherwise, where $a$ and $b$ are distinct constants. For empty $D_{m}$ and $\bar{V}$, no ground instances are in $\operatorname{RCQ}^{w}\left(Q, D_{m}, V\right)$ since $Q\left(I_{1}, I_{2}\right) \neq \emptyset$ for all $\left(I_{1}, I_{2}\right)$, while $\bigcap_{\mathcal{I}^{\prime} \in \operatorname{Ext}\left(I_{1}, I_{2}\right)} Q\left(\mathcal{I}^{\prime}\right)=\emptyset$. In contrast, consider a $c$-instance $\mathcal{T}=\left(T_{1}, T_{2}\right)$, where $T_{1}=(\{(x)\}, \emptyset)$ and $T_{2}=(\{(y)\}, \emptyset)$. Obviously, $\mathcal{T}$ is in $\operatorname{RCQ}^{w}\left(Q, D_{m}, V\right)$ since $Q(\mathcal{T})=$ $\bigcap_{\mathcal{I} \in \operatorname{Mod}(\mathcal{T})} Q(\mathcal{I})=\emptyset=\bigcap_{\mathcal{I} \in \operatorname{Mod}(\mathcal{T}), \mathcal{I}^{\prime} \in \operatorname{Ext}(\mathcal{I})} Q\left(\mathcal{I}^{\prime}\right)$.

This tells us that, from the undecidability of $\operatorname{RCQP}^{w}(\mathrm{FO})$ for ground instances, we cannot conclude the undecidability for $c$-instances. Nevertheless, $\operatorname{RCQP}^{w}\left(\mathcal{L}_{Q}\right)$ becomes trivially decidable when $\mathcal{L}_{Q}$ is $\mathrm{FP}, \mathrm{CQ}, \mathrm{UCQ}$, or $\exists \mathrm{FO}^{+}$, for $c$-instances and for ground instances, in contrast to Theorem 4.5.

Theorem 5.4. $\operatorname{RCQP}^{w}\left(\mathcal{L}_{Q}\right)$ is

- undecidable for ground instances if $\mathcal{L}_{Q}$ is $\mathrm{FO}$, and

-decidable in $O(1)$-time for c-instances and ground instances when $\mathcal{L}_{Q}$ is FP, CQ, UCQ, or $\exists F O^{+}$.

The complexity is unchanged when $D_{m}$ and $V$ are fixed.

Proof. We refer to Appendix A for the proof of this Theorem. In a nutshell, we show that $\mathrm{RCQP}^{w}(\mathrm{FO})$ is undecidable for ground instances by reduction from the satisfiability problem for FO, and give a constructive proof showing that there always exists a database (ground instance) that is weakly complete for $Q$ relative to $\left(D_{m}, V\right)$ when $Q$ is an FP query. From this, it follows that $\operatorname{RCQP}^{w}(\mathrm{FP})$ is trivially decidable.

\subsection{The Minimality Problem in the Weak Model}

In contrast to the strong completeness model, Lemma 4.7 no longer holds in the weak completeness model, that is, to decide whether an instance $I$ is minimal, it does not suffice to inspect $I \backslash\{t\}$ only. 
Example 5.5. Consider a CQ query $Q$ defined on a pair of unary relations $\left(R_{1}, R_{2}\right)$ : $Q(x)=\exists y z\left(R_{1}(y) \wedge R_{2}(z) \wedge x=a\right)$. That is, on an instance $\left(I_{1}, I_{2}\right)$ of $\left(R_{1}, R_{2}\right), Q$ returns $\{(a)\}$ if $I_{1}$ and $I_{2}$ are both nonempty. Consider an instance $\mathcal{I}_{0}=(\{(0)\},\{(1)\})$, an empty set $V$ of CCs, and any master data $D_{m}$. Then, $\mathcal{I}_{0}$ is in $\operatorname{RCQ}^{w}\left(Q, D_{m}, V\right)$. Nevertheless, it is not minimal: the empty instance $(\emptyset, \emptyset)$ of $\left(R_{1}, R_{2}\right)$ is also in $\operatorname{RCQ}^{w}\left(Q, D_{m}, V\right)$. Indeed, $(\{0\}, \emptyset)$ is a partially closed extension of $(\emptyset, \emptyset)$ and $Q(\{0\}, \emptyset)=\emptyset$. Then, $\bigcap_{I^{\prime} \in \operatorname{Ext}((\emptyset, \emptyset))}=\emptyset$; thus, $(\emptyset, \emptyset)$ is in $\operatorname{RCQ}^{w}\left(Q, D_{m}, V\right)$. However, removing one tuple from $\mathcal{I}_{0}$ does not make it a weakly complete instance, that is, a counterexample to the minimality of $\mathcal{I}_{0}$ cannot be found by removing only one tuple from $\mathcal{I}_{0}$.

In the weak model, the minimality analysis is quite different from its counterpart in the strong model (Theorem 4.8). (a) The absence of missing values does not simplify the analysis, as opposed to their counterparts in the strong model $\left(D_{2}^{p}\right.$ for ground instances vs. $\Pi_{3}^{p}$ for $c$-instances). (b) It is much easier to check $\operatorname{MINP}^{w}(\mathrm{CQ})$ than $\operatorname{MINP}^{w}(\mathrm{UCQ})$ (coDP-complete vs. $\Pi_{4}^{p}$-complete), whereas $\mathrm{MINP}^{s}(\mathrm{CQ})$ and $\mathrm{MINP}^{s}(\mathrm{UCQ}$ ) have the same complexity. Recall that coDP $=$ NP $\cup$ coNP (see Papadimitriou [1994]).

Theorem 5.6. For c-instances and ground instances. $\operatorname{MINP}^{w}\left(\mathcal{L}_{Q}\right)$ is

—undecidable when $\mathcal{L}_{Q}$ is $\mathrm{FO}$,

- conEXPTIME-complete when $\mathcal{L}_{Q}$ is $F P$,

$-\Pi_{4}^{p}$-complete when $\mathcal{L}_{Q}$ is $U C Q$ or $\exists F O^{+}$, and

- coDP-complete when $\mathcal{L}_{Q}$ is $C Q$.

Proof. We show that $\operatorname{MINP}^{w}\left(\mathcal{L}_{Q}\right)$ is undecidable when $\mathcal{L}_{Q}$ is FO, coneXPTIME-complete when $\mathcal{L}_{Q}$ is $\mathrm{FP}$, and $\Pi_{4}^{p}$-complete when $\mathcal{L}_{Q}$ is UCQ or $\exists \mathrm{FO}^{+}$. When $\mathcal{L}_{Q}$ is $\mathrm{CQ}$, the problem is shown to be coDP-complete. All lower bounds remain intact when only ground instances are considered.

(1) When $\mathcal{L}_{Q}$ is FO. It suffices to show that $\operatorname{MINP}^{w}(\mathrm{FO})$ is undecidable for ground instances. More specifically, it suffices to show that it is undecidable to determine, given an FO query $Q$, master data $D_{m}$ and a set $V$ of CCs, whether $\mathcal{I}_{\emptyset}$ is in $\operatorname{RCQ}^{w}\left(Q, D_{m}, V\right)$, where $\mathcal{I}_{\emptyset}$ is the empty database instance of the schema over which $Q$ is defined. This is because if $\mathcal{I}_{\emptyset}$ is in $\operatorname{RCQ}^{w}\left(Q, D_{m}, V\right)$, then it is a minimum instance complete for $Q$ relative to $\left(D_{m}, V\right)$. The undecidability of this problem has already been verified in the proof of Theorem 5.4.

(2) When $\mathcal{L}_{Q}$ is FP. We show that MINP ${ }^{w}(\mathrm{FP})$ is conEXPTIME-hard for ground instances and provide a coNEXPTIME algorithm for deciding $\operatorname{MINP}^{w}(\mathrm{FP})$ for $c$-instances.

Lower bound. We show that, for ground instances, MINP ${ }^{w}(\mathrm{FP})$ is conEXPTIME-hard by reduction from the SUCCINCT-TAUT problem (see a description of the problem in the proof of Theorem 5.1 (2)). Given an instance of the latter problem, we define the same database schemas $\mathcal{R}$ and $\mathcal{R}_{m}$, ground instance $\mathcal{I}$ of $\mathcal{R}$, set $V$ of $\mathrm{CCs}$, master data $D_{m}$ of $\mathcal{R}_{m}$, and FP query $Q$ as their counterparts in the proof of Theorem 5.1 (2). We show that $C$ is a tautology if and only if $\mathcal{I}$ is the minimal in $\operatorname{RCQ}^{w}\left(Q, D_{m}, V\right)$.

$\Rightarrow$ Suppose that $C$ is a tautology. We first show that $\mathcal{I} \in \operatorname{RCQ}^{w}\left(Q, D_{m}, V\right)$, that is, $Q(\mathcal{I})=\bigcap_{\mathcal{I}^{\prime} \in \operatorname{Ext}(\mathcal{I})} Q\left(\mathcal{I}^{\prime}\right)$. As shown in the proof of Theorem $5.1(2), Q(\mathcal{I})$ returns all $\vec{w} \in\{0,1\}^{n}$ since $C$ is a tautology. Moreover, the only extension $\mathcal{I}^{\prime}$ of $\mathcal{I}$ is $\left\{t, t^{\prime}\right\}$, where $t$ is in $\mathcal{I}$ and $t^{\prime}$ is the same as $t$ except that $t^{\prime}\left[A_{0}\right]=0$ while $t\left[A_{0}\right]=1$, and $Q\left(\mathcal{I}^{\prime}\right)$ returns all $\vec{w} \in\{0,1\}^{n}$ as well. Then, $\mathcal{I} \in \operatorname{RCQ}^{w}\left(Q, D_{m}, V\right)$. One can verify that $\emptyset \notin \operatorname{RCQ}^{w}\left(Q, D_{m}, V\right)$. Indeed, as discussed earlier, $\bigcap_{\left(\mathcal{I}^{\prime}, D_{m}\right) \models V} Q\left(\mathcal{I}^{\prime}\right)=\{0,1\}^{n}$. Hence, $\mathcal{I}$ is weakly complete and minimal. 
$\Leftarrow$ Conversely, if $C$ is not a tautology, then, by the proof of Theorem $5.1(2), \mathcal{I}$ is not even in $\operatorname{RCQ}^{w}\left(Q, D_{m}, V\right)$, hence is not minimal.

Upper bound. We provide a coNEXPTIME algorithm that, given a $c$-instance $\mathcal{T}$, master data $D_{m}$, an FP query $Q$, and a set $V$ of CCs, the algorithm returns "yes" if $\mathcal{T}$ is weakly complete and minimal. The algorithm works as follows:

(1) Check whether $\mathcal{T} \in \mathrm{RCQ}^{w}\left(Q, D_{m}, V\right)$. This is a coneXPTIME process by Theorem 5.1(2). If so, continue; otherwise, return "no."

(2) For each $\Delta \subseteq \mathcal{T}, \Delta \neq \emptyset$, check whether $\mathcal{T} \backslash \Delta \in \mathrm{RCQ}^{w}\left(Q, D_{m}, V\right)$. If such a $\Delta$ is found, return "no"; otherwise, return "yes." The enumeration of the subsets $\Delta$ and checking whether $\mathcal{T} \backslash \Delta \in \mathrm{RCQ}^{w}\left(Q, D_{m}, V\right)$ are again in conEXPTIME.

Hence, $\mathrm{MINP}^{w}(\mathrm{FP})$ is in conEXPTIME. The correctness of the algorithm is immediate.

(3) When $\mathcal{L}_{Q}$ is UCQ or $\exists \mathrm{FO}^{+}$. We next show that $\operatorname{MINP}^{w}\left(\mathcal{L}_{Q}\right)$ is $\Pi_{4}^{p}$-complete when $\mathcal{L}_{Q}$ is $\mathrm{UCQ}$ or $\exists \mathrm{FO}^{+}$. It suffices to show that MINP ${ }^{w}(\mathrm{UCQ})$ is $\Pi_{4}^{p}$-hard, and $\mathrm{MINP}^{w}\left(\exists \mathrm{FO}^{+}\right)$is in $\Pi_{4}^{p}$. The lower bound holds when considering ground instances only.

Lower bound. We show the $\Pi_{4}^{p}$-hardness by reduction from the $\forall^{*} \exists^{*} \forall^{*} \exists^{*}$ 3SAT problem, which is known to be $\Pi_{4}^{p}$-complete (see Papadimitriou [1994]). The $\forall^{*} \exists^{*} \forall^{*} \exists^{*} 3$ sat problem is to determine, given a sentence $\varphi=\forall X \exists Y \forall Z \exists W \psi$, whether $\varphi$ is true. Here, $X=$ $\left\{x_{1}, \ldots, x_{n}\right\}, Y=\left\{y_{1}, \ldots, y_{m}\right\}, Z=\left\{z_{1}, \ldots, z_{k}\right\}, W=\left\{w_{1}, \ldots, w_{p}\right\}$, and $\psi$ is an instance of 3sat. Given an instance $\varphi=\forall X \exists Y \forall Z \exists W \psi$ of the $\forall^{*} \exists^{*} \forall^{*} \exists^{*}$ 3saT problem, where $\psi=$ $C_{1} \wedge \cdots \wedge C_{r}$, we define a database schema $\mathcal{R}$, a ground instance $\mathcal{I}_{0}$ of $\mathcal{R}$, master data $D_{m}$, a set $V$ of CCs, and a query $Q$ in UCQ, such that $\varphi$ is true if and only if either $\mathcal{I}_{0}$ is not in $\operatorname{RCQ}^{w}\left(Q, D_{m}, V\right)$ or $\mathcal{I}_{0} \in \mathrm{RCQ}^{w}\left(Q, D_{m}, V\right)$, but it is not minimal.

(a) The database schema $\mathcal{R}$ consists of the following six relation schemas: $R_{(0,1)}(X)$, $R_{\vee}\left(A_{1}, A_{2}, B\right), R_{\wedge}\left(A_{1}, A_{2}, B\right), R_{\neg}\left(A_{1}, A_{2}\right), R_{X}(\mathrm{id}, X)$, and $R_{Z}\left(Z_{1}, \ldots, Z_{k}\right)$. Instances of $R_{X}(\mathrm{id}, X)$ are to encode truth assignments of $X$, and instances of $R_{Z}$ are singleton sets, each encoding a truth assignment of $Z$. We let $\mathcal{I}_{0}=\left(I_{X}, I_{(0,1)}, I_{\vee}, I_{\wedge}, I_{\neg}, I_{Z}\right)$, where $I_{X}=\{(1,0),(1,1), \ldots,(n, 0),(n, 1)\}, I_{Z}=\emptyset$, and $I_{(0,1)}, I_{\vee}, I_{\wedge}$, and $I_{\neg}$ are as shown in Figure 2 .

(b) The master data $D_{m}$ and CCs $V$ ensure the following: (i) any instance $I_{X}^{\prime}$ of $R_{X}$ satisfies $I_{X}^{\prime} \subseteq I_{X}$; (ii) any instance $I_{Z}^{\prime}$ of $R_{Z}$ consists of a single tuple with values taken from $\{0,1\}$; and (iii) instances of $R_{(0,1)}, R_{\vee}, R_{\wedge}$, and $R_{\neg}$ are subsets of $I_{(0,1)}, I_{\vee}, I_{\wedge}$, and $I_{\neg}$, respectively. Clearly, $\left(\mathcal{I}, D_{m}\right) \models V$.

(c) The query $Q$ is defined as $Q_{1} \cup \cdots \cup Q_{2 n+12} \cup P_{1} \cup P_{2} \cup P_{3}$, where the $Q_{i}$ s guarantee the existence of certain tuples in the query result when the input instance consists of at least $i$ tuples, for $i \in[1,2 n+12]$; query $P_{1}$ generates an additional tuple when the instance of $R_{X}$ contains a proper truth assignment of $X$; query $P_{2}$ is to eliminate the effect on the certain answers of extensions that do not correspond to proper truth assignments of $X$; and, finally, $P_{3}$ generates truth assignments of $Y$ that satisfy certain properties related to $\varphi$. As we will see here, $\mathcal{I}_{0} \in \operatorname{RCQ}^{w}\left(Q, D_{m}, V\right)$, but it is only minimal when there does not exist a proper subset $I_{X}^{-}$of $I_{X}$ such that $\mathcal{I}_{0}^{-}=\left(I_{X}^{-}, I_{(0,1)}, I_{\vee}, I_{\wedge}, I_{\neg}, \emptyset\right) \in \mathrm{RCQ}^{w}\left(Q, D_{m}, V\right)$. Furthermore, $Q$ is defined in such a way that only $I_{X}^{-}$that encode valid truth assignments of $X$ need to be considered. In particular, $\mathcal{I}_{0}^{-} \in \mathrm{RCQ}^{w}\left(Q, D_{m}, V\right)$ if and only if the truth assignment $\mu_{X}$ encoded in $I_{X}^{-}$is such that there does not exist a $\mu_{Y}$ of $Y$ such that, for every $\mu_{Z}$ of $Z, \exists W \psi\left(\mu_{X}, \mu_{Y}, \mu_{Z}, W\right)$ evaluates to true. In other words, $\mathcal{I}_{0}^{-} \in \operatorname{RCQ}^{w}\left(Q, D_{m}, V\right)$ if and only if $\varphi$ is false. Consequently, $\mathcal{I}_{0}$ is minimal if and only if $\varphi$ is true. In the rest of the lower bound proof, we use 
$\mathcal{I}$ and $\mathcal{I}^{\prime}$ to range over the instances of $\mathcal{R}, \mathcal{I}^{-}$, and $\left(\mathcal{I}^{\prime}\right)^{-}$to denote subinstances (proper subsets) of $\mathcal{I}$ and $\mathcal{I}^{\prime}$, and $\mathcal{I}^{+}$and $\left(\mathcal{I}^{\prime}\right)^{+}$to denote extensions of $\mathcal{I}$ and $\mathcal{I}^{\prime}$, respectively.

We next explain the disjuncts in $Q$ in more detail. All queries have output arity $m$, the number of variables in $Y$. Observe that the maximal size of partially closed instances is $2 n+13$, that is, there are at most $2 n$ tuples in instances of $R_{X}, 12$ tuples in the instances corresponding to $R_{(0,1)}, R_{\vee}, R_{\wedge}$, and $R_{\neg}$, and at most one tuple in instances of $R_{Z}$.

For $i \in[1,2 n+12]$, we define $Q_{i}(\vec{u})$ as a UCQ that returns $\vec{a}_{i}=\left(a_{i}, \ldots, a_{i}\right)$ whenever the input instance has size at least $i$. Here, $a_{i}$ is a fresh new constant not used anywhere else. Clearly, such a query can be expressed in UCQ by using $\neq$.

Consider $Q^{\prime}=Q_{1} \cup \cdots \cup Q_{2 n+12}$ and any instance $\mathcal{I}$ of $\mathcal{R}$ of size $i$. Then, $Q^{\prime}(\mathcal{I})=$ $\left\{\vec{a}_{1}, \ldots, \vec{a}_{i}\right\}$. However, for any extension $\mathcal{I}^{+}$of $\mathcal{I}$ (i.e., for $\mathcal{I}^{+} \in \operatorname{Ext}(\mathcal{I})$ ), we have that $Q^{\prime}(\mathcal{I}) \subsetneq Q^{\prime}\left(\mathcal{I}^{+}\right)$since the latter surely contains $\left\{\vec{a}_{1}, \ldots, \vec{a}_{i}, \vec{a}_{i+1}\right\}$. In other words, if we were to use only $Q^{\prime}$ instead of $Q$, no strict subinstance $\mathcal{I}^{-}$of $\mathcal{I}_{0}$ can be in $\mathrm{RCQ}^{w}\left(Q^{\prime}, D_{m}, V\right)$. We will see shortly how the additional query $P_{1}$ in $Q$ provides the opportunity for specific subinstances of $\mathcal{I}$, that is, those that correspond to valid truth assignments of $X$, to be in $\operatorname{RCQ}^{w}\left(Q, D_{m}, V\right)$. Observe that $Q^{\prime}\left(\mathcal{I}_{0}\right)=\left\{\vec{a}_{1}, \ldots, \vec{a}_{2 n+12}\right\}$. Similarly, $Q^{\prime}\left(\mathcal{I}_{0}^{+}\right)=\left\{\vec{a}_{1}, \ldots, \vec{a}_{2 n+12}\right\}$ for any extension $\mathcal{I}_{0}^{+}$of $\mathcal{I}_{0}$. Indeed, $Q^{\prime}$ stops adding fresh tuples to the query result once the instance grows in size beyond $2 n+12$. Hence, $Q^{\prime}$ helps us ensure that $\mathcal{I}_{0} \in \mathrm{RCQ}^{w}\left(Q, D_{m}, V\right)$.

We next define the queries $P_{1}, P_{2}$, and $P_{3}$. First, we let

$$
P_{1}(\vec{u})=\left(\exists \vec{x} \bigwedge_{i \in[1, n]} R_{X}\left(i, x_{i}\right)\right) \wedge Q_{\text {all }} \wedge \vec{u}=\vec{a}_{n+13},
$$

where $Q_{\text {all }}$ is to ensure that all the tuples in $I_{(0,1)}, I_{\neg}, I_{\vee}$, and $I_{\wedge}$ are in place. That is, query $P_{1}$ puts $\vec{a}_{n+13}$ into the query result on instances $\mathcal{I}^{\prime}$ of $\mathcal{R}$ for which $I_{X}^{\prime}$ contains a truth assignment $\mu_{X}$ of $X$, that is, when $I_{X}^{\prime}$ contains tuples of the form $(i, v)$ for each $i \in[1, n]$, and when all instances encoding Boolean domain and operations are present. Observe that $P_{1}$ has an effect only when $\mathcal{I}^{\prime}$ has size $n+12$ (when its $R_{X}$ instance encodes a valid truth assignment for $X$ ). $\vec{a}_{n+13}$ is already in the query result for larger instances because of the $Q_{j}$ s described earlier. On the other hand, it cannot affect instances $\mathcal{I}^{\prime}$ of smaller size. $I_{X}^{\prime}$ must contain at least $n$ tuples and all 12 tuples in $I_{(0,1)}, I_{\vee}, I_{\wedge}$, and $I_{\neg}$ must be present.

It is easily verified that, for $Q^{\prime \prime}=Q^{\prime} \cup P_{1}, \mathcal{I}_{0}^{-} \in \mathrm{RCQ}^{w}\left(Q^{\prime \prime}, D_{m}, V\right)$ if and only if either (a) $\mathcal{I}=\mathcal{I}_{0}$ or (b) $\mathcal{I}_{0}^{-}$consists of precisely $n+12$ tuples and satisfies the condition in $P_{1}$. We refer to such subinstances $\mathcal{I}_{0}^{-}$of $\mathcal{I}_{0}$ as weakly complete candidates. That is, we use $Q^{\prime}$ and $P_{1}$ to distinguish weakly complete candidates. Observe that the certain answers of $Q^{\prime \prime}$ on extensions of $\mathcal{I}_{0}^{-}$is equal to $\left\{\vec{a}_{1}, \ldots, \vec{a}_{n+12}, \vec{a}_{n+13}\right\}$, which equals $Q^{\prime \prime}\left(\mathcal{I}_{0}^{-}\right)$.

What remains to show is that no weakly complete candidates can be weakly complete if and only if $\varphi$ is true. For if this holds, $\mathcal{I}_{0}$ is minimal in $\operatorname{RCQ}^{w}\left(Q, D_{m}, V\right)$ if and only if $\varphi$ is true, as desired. To show this, we need the two additional queries $P_{2}$ and $P_{3}$, which are defined as follows.

Query $P_{2}$ is to ensure that, for any extension $\left(\mathcal{I}_{0}^{-}\right)^{+}$of weakly complete candidates $\mathcal{I}_{0}^{-}$, if its $R_{X}$ instance $\left(I_{X}^{\prime}\right)^{+}$does not encode a truth assignment of $X$, then $\left(\mathcal{I}_{0}^{-}\right)^{+}$does not affect the certain-answer result. More specifically, $P_{2}$ is a disjunction of $n$ queries $P_{2, i}(\vec{y})=R_{X}(i, 0) \wedge R_{X}(i, 1) \wedge R_{(0,1)}\left(y_{1}\right) \wedge \cdots \wedge R_{(0,1)}\left(y_{m}\right)$. That is, whenever $P_{2}$ is applied on an instance $\left(\mathcal{I}_{0}^{-}\right)^{+}$such that $\left(I_{X}^{\prime}\right)^{+}$contains two possible values for a variable $x_{i}$ (encoded by $(i, 0)$ and $(i, 1))$, it puts all truth assignments of $Y$ in the query result. We denote by $F_{Y}$ the set of all possible truth assignments of $Y$.

Consider $Q^{\prime \prime}=Q^{\prime} \cup P_{2}$. Observe that $Q^{\prime \prime}\left(\mathcal{I}_{0}\right)=\left\{\vec{a}_{1}, \ldots, \vec{a}_{2 n+12}\right\} \cup F_{Y}=Q^{\prime \prime}\left(\mathcal{I}_{0}^{+}\right)$for any extension of $\mathcal{I}_{0}^{+}$of $\mathcal{I}_{0}$. Hence, $\mathcal{I}_{0} \in \mathrm{RCQ}^{w}\left(Q^{\prime \prime}, D_{m}, V\right)$. Similarly, for a weakly complete 
candidate $\mathcal{I}_{0}^{-}, Q^{\prime \prime}\left(\mathcal{I}_{0}^{-}\right)=\left\{\vec{a}_{1}, \ldots, \vec{a}_{n+13}\right\}=\bigcap_{\left(\mathcal{I}_{0}^{-}\right)^{+} \in \operatorname{Ext}\left(\mathcal{I}_{0}^{-}\right)} Q^{\prime \prime \prime}\left(\left(\mathcal{I}_{0}^{-}\right)^{+}\right)$. This follows from the fact that $\operatorname{Ext}\left(\mathcal{I}_{0}^{-}\right)$contains at least one instance $\mathcal{I}_{1}$ of size $n+13$ on which $P_{2}$ does not produce $F_{Y}$. For example, $\mathcal{I}_{1}$ could be $\mathcal{I}_{0}^{-}$extended with an additional tuple in its $R_{Z}$ instance $I_{Z}^{\prime}$. Since $Q^{\prime \prime}\left(\mathcal{I}_{1}\right)=Q\left(\mathcal{I}_{0}^{-}\right)=\left\{\vec{a}_{1}, \ldots, \vec{a}_{n+13}\right\} \subseteq \bigcap_{\mathcal{I}^{\prime} \in \operatorname{Ext}\left(\mathcal{I}_{0}^{-}\right)} Q^{\prime \prime \prime}\left(\mathcal{I}^{\prime}\right)$ and $\mathcal{I}_{1} \in \operatorname{Ext}\left(\mathcal{I}_{0}^{-}\right)$, we may conclude that $P_{2}$ alone does not prevent weakly complete candidates to be in $\mathrm{RCQ}^{w}\left(Q^{\prime \prime}, D_{m}, V\right)$. The relevance of $P_{2}$ comes only in play together with query $P_{3}$, which we define next.

More specifically,

$$
P_{3}(\vec{y})=\exists \vec{x}, \vec{z}, \vec{w}\left(Q_{X}(\vec{x}) \wedge Q_{Y}(\vec{y}) \wedge R_{Z}(\vec{z}) \wedge Q_{W}(\vec{w}) \wedge Q_{\psi}(\vec{x}, \vec{y}, \vec{z}, \vec{w}, 1)\right),
$$

where $Q_{X}(\vec{x})=\bigwedge_{i \in[1, n]} R_{X}\left(i, x_{i}\right)$, and $Q_{Y}(\vec{y})$ and $Q_{W}(\vec{w})$ generate all $k$ and $p$ binary tuples by means of Cartesian products of $R_{(0,1)}$. Furthermore, $Q_{\psi}(\vec{x}, \vec{y}, \vec{z}, \vec{w}, v)$ is a CQ query that encodes the truth value of $\psi\left(\mu_{X}, \mu_{Y}, \mu_{Z}, \mu_{W}, v\right)$ for given truth assignment $\mu_{X}$ of $X, \mu_{Y}$ of $Y, \mu_{Z}$ of $Z$, and $\mu_{W}$ of $W$ as encoded by $\vec{x}, \vec{y}, \vec{z}$, and $\vec{w}$, respectively. In other words, $v=1$ if $\psi\left(\mu_{X}, \mu_{Y}, \mu_{Z}, \mu_{W}, v\right)$ holds and $v=0$ otherwise. Query $Q_{\psi}$ is encoded by means of $R_{(0,1)}, R_{\vee}, R_{\wedge}$, and $R_{\neg}$, as before.

We now have that $Q=Q^{\prime \prime} \cup P_{3}$. Observe that $Q\left(\mathcal{I}_{0}\right)=Q^{\prime \prime}\left(\mathcal{I}_{0}\right)$. Furthermore, since $Q^{\prime \prime \prime}\left(\mathcal{I}_{0}\right)$ already contains $F_{Y}$, no further tuples can be added to the query result in any extension of $\mathcal{I}_{0}$. Hence, $\mathcal{I}_{0}$ remains a weakly complete database for $Q, D_{m}$, and $V$.

We next investigate the impact of $P_{3}$ on weakly complete candidates $\mathcal{I}_{0}^{-}$. Since $I_{Z}=\emptyset$ in $\mathcal{I}_{0}, I_{Z}^{\prime}=\emptyset$ in any weakly complete candidate $\mathcal{I}_{0}^{-}$; therefore, $P_{3}$ does not add additional tuples to $Q^{\prime \prime \prime}\left(\mathcal{I}_{0}^{-}\right)$, that is, $Q\left(\mathcal{I}_{0}^{-}\right)=Q^{\prime \prime}\left(\mathcal{I}_{0}^{-}\right)$. On the other hand, consider an extension $\left(\mathcal{I}_{0}^{-}\right)^{+}$of $\mathcal{I}_{0}^{-}$on which $P_{2}$ does not apply (otherwise, $P_{2}$ would already have added $F_{Y}$ to the query result, hence $P_{3}$ does not have an impact). Recall that such extensions exist by simply adding a tuple to $I_{Z}^{\prime}$. Then, $P_{3}\left(\left(\mathcal{I}_{0}^{-}\right)^{+}\right)$is either (a) empty, in which case $\bigcap_{\left(\mathcal{I}_{0}^{-}\right)^{+} \in \operatorname{Ext}\left(\mathcal{I}_{0}^{-}\right)} Q\left(\left(\mathcal{I}_{0}^{-}\right)^{+}\right)=\left\{\vec{a}_{1}, \ldots, \vec{a}_{n+13}\right\}=Q\left(\mathcal{I}_{0}^{-}\right)$, or (b) $P_{3}\left(\left(\mathcal{I}_{0}^{-}\right)^{+}\right)$returns $F_{Y}^{\prime}$, where $F_{Y}^{\prime}$ consists of all truth assignments of $Y$ that satisfy the condition in $P_{3}$. It is easily verified that $\bigcap_{\left(\mathcal{I}_{0}^{-}\right)^{+} \in \operatorname{Ext}\left(\mathcal{I}_{0}^{-}\right)} Q\left(\left(\mathcal{I}_{0}^{-}\right)^{+}\right)=\left\{\vec{a}_{1}, \ldots, \vec{a}_{n+13}\right\} \cup C_{Y}$, where $C_{Y}$ denotes the set of truth assignments returned by $P_{3}$ on all extensions $\left(\mathcal{I}_{0}^{-}\right)^{+}$of $\mathcal{I}_{0}^{-}$. Hence, the weakly complete candidate $\mathcal{I}_{0}^{-}$is weakly complete if and only if $C_{Y}$ is empty. Recall that $Q\left(\mathcal{I}_{0}^{-}\right)=\left\{\vec{a}_{1}, \ldots, \vec{a}_{n+13}\right\}$. That is, for the truth assignment $\mu_{X}$ encoded in $I_{X}^{\prime}$, there is no truth assignment $\mu_{Y}$ of $Y$ such that, for all truth assignments $\mu_{Z}$ of $Z$ (note that all $\mu_{Z}$ are considered as a tuple in $I_{Z}^{\prime \prime}$ in some extension $\left(\mathcal{I}_{0}^{-}\right)^{+}$of $\mathcal{I}_{0}^{-}$), there exists a truth assignment $\mu_{W}$ of $W$ that makes $\psi$ true. That is, a weakly complete candidate is actually in $\operatorname{RCQ}^{w}\left(Q, D_{m}, V\right)$ if and only if $\varphi$ is false, as desired. As a result, $\mathcal{I}$ is a minimal instance in $\operatorname{RCQ}^{w}\left(Q, D_{m}, V\right)$ if and only if $\varphi$ is true.

Upper bound. We show that $\operatorname{MINP}^{w}\left(\exists \mathrm{FO}^{+}\right)$is in $\Pi_{4}^{p}$ by providing an $\Sigma_{4}^{p}$-algorithm that decides the complement problem. That is, the algorithm returns "yes" if, for a given $c$-instance $\mathcal{T}$, master data $D_{m}$, a set $V$ of CCs, and an $\exists \mathrm{FO}^{+}$query $Q, \mathcal{T}$ is not a minimal $c$-instance that is weakly complete for $Q$ relative to $\left(D_{m}, V\right)$, and "no" otherwise. The algorithm does the following:

(1) Check whether $\mathcal{T} \notin \mathrm{RCQ}^{w}\left(Q, D_{m}, V\right)$. If so, then return "yes." Otherwise, continue. By Theorem 5.1(3), this step is in $\Sigma_{3}^{p}$.

(2) We guess $\Delta \subseteq \mathcal{T}$ and make a call to a $\Pi_{3}^{p}$-oracle, to check whether $\mathcal{T} \backslash \Delta \in$ $\mathrm{RCQ}^{w}\left(Q, D_{m}, \bar{V}\right)$. If not, reject the current guess. Otherwise, return "yes."

Hence, the overall complexity of the algorithm is $\mathrm{NP}^{\Sigma_{3}^{p}}$ or $\Sigma_{4}^{p}$; therefore, $\operatorname{MINP}\left(\exists \mathrm{FO}^{+}\right)$is in $\Pi_{4}^{p}$. The correctness of the algorithm is immediate. 
(4) When $\mathcal{L}_{Q}$ is $\mathrm{CQ}$. We show that $\operatorname{MINP}^{w}(\mathrm{CQ})$ is coDP-complete for ground instances. By Lemma 3.2, we assume without loss of generality that $\mathcal{T}$ is defined over a single relation schema. When $\mathcal{L}_{Q}$ is $\mathrm{CQ}$, the minimal weakly complete databases are rather restrictive, as verified by the following lemma:

Lemma 5.7. Given a $C Q$ query $Q$, master data $D_{m}$, a set $V$ of $C C s$, and a $c$ instance $\mathcal{T}, \mathcal{T}$ is a minimal instance in $\operatorname{RCQ}^{w}\left(Q, D_{m}, V\right)$ if and only if either $\mathcal{T}=\varnothing$ is in $\operatorname{RCQ}^{w}\left(Q, D_{m}, V\right)$, or when $\emptyset \notin \operatorname{RCQ}^{w}\left(Q, D_{m}, V\right),|\mathcal{T}|$ is a singleton set, and $\operatorname{Mod}\left(\mathcal{T}, D_{m}, V\right) \neq \emptyset$.

Proof. Let $\left(T_{Q}, u_{Q}\right)$ denote the tableau representation of $Q$. We distinguish between the following two cases: (i) $\emptyset \in \mathrm{RCQ}^{w}\left(Q, D_{m}, V\right)$ and (ii) $\emptyset \notin \mathrm{RCQ}^{w}\left(Q, D_{m}, V\right)$. Clearly, in case (i), $\mathcal{T}$ is a minimal instance in $\operatorname{RCQ}^{w}\left(Q, D_{m}, V\right)$ if and only if $\mathcal{T}=\emptyset$. Suppose that case (ii) holds. We then show that $\{\tau\} \in \operatorname{RCQ}^{w}\left(Q, D_{m}, V\right)$ for every $\tau \in \mathcal{T}$, where $\operatorname{Mod}\left(\tau, D_{m}, V\right) \neq \emptyset$. Hence, one can readily verify that in case (ii), $\mathcal{T}$ is a minimal instance in $\operatorname{RCQ}^{w}\left(Q, D_{m}, V\right)$ if and only if $|\mathcal{T}|=1$ and $\operatorname{Mod}\left(\mathcal{T}, D_{m}, V\right) \neq \emptyset$.

Suppose that $\emptyset \notin \mathrm{RCQ}^{w}\left(Q, D_{m}, V\right)$. Then, $\bigcap_{\left(\{t\}, D_{m}\right)=V} Q(\{t\}) \neq \varnothing$. Once $\bigcap_{\left(\{t\}, D_{m}\right) \models V}$ $Q(\{t\})=\emptyset$, then $\bigcap_{\mathcal{I}^{\prime} \in \operatorname{Ext}(\emptyset)} Q\left(\mathcal{I}^{\prime}\right)=\varnothing=Q(\emptyset)$; thus, $\emptyset \in \mathrm{RCQ}^{w}\left(Q, D_{m}, V\right)$, which contradicts the assumption. Moreover, it is easy to verify that $Q(\{t\})$ consists of a single tuple, and all $Q(\{t\})$ must return the same answer tuple, say, $u$. Let $\tau$ be a tuple in $\mathcal{T}$ such that $\operatorname{Mod}\left(\tau, D_{m}, V\right) \neq \varnothing$. First, note that $\operatorname{Mod}\left(\tau, D_{m}, V\right)$ is a subset of $\left\{t \mid\left(\{t\}, D_{m}\right) \models V\right\}$ and, for each $t \in \operatorname{Mod}\left(\tau, D_{m}, V\right)$, we have that $Q(\{t\})=u$. We show that $\{\tau\}$ is in $\operatorname{RCQ}^{w}\left(Q, D_{m}, V\right)$. We only need to show that the intersection of $Q(\{t, s\})$, where $t \in \operatorname{Mod}\left(\tau, D_{m}, V\right)$ and $\left(\{t, s\}, D_{m}\right) \models V$, is equal to $u$. In fact, we show a stronger result: $Q(\{t, s\})=u$ for each $t$ and $s$ as before. Suppose, by contradiction, that there exists a valuation $\mu^{\prime}$ of $T_{Q}$ with values from $t$ and $s$ such that $\mu^{\prime}\left(u_{Q}\right) \neq u$. However, every variable $x \in u_{Q}$ such that $\mu^{\prime}(x) \neq u[x]$ is witnessed by an attribute either in $t$ or $s$ (or both), as specified by $\mu^{\prime}$. Observe, however, that also $\left(\{s\}, D_{m}\right) \models V$, thus also $Q(\{(s)\})=u$. This implies, in turn, that $\mu^{\prime}(x)$ is already witnessed by a valuation of $Q$ with values in $t$, or by a valuation of $Q$ with values in $s$, both of which result in $u$. Thus, $\mu^{\prime}\left(u_{Q}\right) \neq u$ cannot be true. A contradiction.

From this lemma, it follows that we only need to consider $c$-instances $\mathcal{T}$ such that either $\mathcal{T}=\emptyset$ or $|\mathcal{T}|=1$. Furthermore, for $\mathcal{T}$ with $|\mathcal{T}| \leq 1$, the problem of testing minimality reduces to testing whether $\emptyset \in \operatorname{RCQ}^{w}\left(Q, D_{m}, \bar{V}\right)$.

Lower bound. We show that, for ground instances, MINP ${ }^{w}(\mathrm{CQ})$ is coDP-hard by reduction from the complement of the SAT-UNSAT problem, which is DP-complete (see Papadimitriou [1994]). An instance of SAT-UNSAT is to determine whether, for a pair of 3sat-instances $\left(\phi, \phi^{\prime}\right), \phi$ is satisfiable and $\phi^{\prime}$ is not satisfiable. Here, $\phi=C_{1} \wedge \cdots \wedge C_{r}$ and $\phi^{\prime}=C_{1}^{\prime} \wedge \cdots \wedge C_{s}^{\prime}$, that is, for each $i \in[1, r]$ (resp. $i \in[1, s]$ ), clause $C_{i}$ (resp. $C_{i}^{\prime}$ ) is of the form $\ell_{1}^{i} \vee \ell_{2}^{i} \vee \ell_{3}^{i}$, where, for each $l \in[1,3], \ell_{l}^{i}$ is either a variable or the negation of a variable in $X=\left\{x_{1}, \ldots, x_{n}\right\}$.

Given an instance of the latter problem, we define a database schema $\mathcal{R}$, a ground instance $I$ of $\mathcal{R}$, master data $D_{m}$, a set $V$ of CCs, and a CQ query $Q$ such that $I$ is a minimal weakly complete instance for $Q$ relative to $\left(D_{m}, V\right)$ if and only if $\phi$ is not satisfiable or $\phi^{\prime}$ is satisfiable.

(a) The database schema $\mathcal{R}$ consists of a single relation $R\left(X_{1}, \ldots, X_{n}, X_{1}^{\prime}, \ldots, X_{n}^{\prime}, Y\right)$, and we set the instance $I$ to be the empty set.

(b) The master data $D_{m}$ consists of two relations $\left(I_{(0,1)}=\{(0),(1)\}, I_{\emptyset}=\emptyset\right)$.

(c) The set $V$ of CCs consists of the following: (i) a constraint enforcing that every attribute in $R$ takes values from $I_{(0,1)}$; (ii) for each clause $C_{i}$, for $i \in[1, r]$, and each 
truth assignment $\mu_{X}$ of the variables in $C_{i}$ that makes $C_{i}$ false, we add a selection condition of the form $\sigma_{\mu_{X}}(R) \subseteq I_{\emptyset}$, ensuring that the projection of $R$ on $X_{1}, \ldots, X_{n}$ contains no tuples satisfying the selection condition. For example, for $C=\bar{x}_{1} \vee \bar{x}_{2} \vee x_{3}$ and $\mu_{X}=\left\{x_{1} \mapsto 1, x_{2} \mapsto 1, x_{3} \mapsto 0\right\}$, we have the constraint $\sigma_{X_{1}=1 \wedge X_{2}=1 \wedge X_{3}=0}(R) \subseteq I_{\varnothing}$; and, finally, (iii) for each $C_{i}^{\prime}$, for $i \in[1, s]$, we add similar constraints to ensure that no tuples in $R$ satisfy $C_{i}^{\prime}$. In addition, these constraints always include $Y=1$. For example, for $C_{i}^{\prime}=x_{3} \vee x_{4} \vee \bar{x}_{5}$ and $\mu_{X}=\left\{x_{3} \mapsto 1, x_{4} \mapsto 0, x_{5} \mapsto 1\right\}$, we have the constraint $\sigma_{Y=1 \wedge X_{3}=1 \wedge X_{4}=0 \wedge X_{5}=1}(R) \subseteq I_{\varnothing}$; note that there are $r+s$ such CC's. Observe the following: (i) $\left\{t[Y] \mid\left(\{t\}, D_{m}\right) \models V\right\}$ is empty if and only if $\phi$ is not satisfiable; (ii) it is $\{(0)\}$ if $\phi$ is satisfiable and $\phi^{\prime}$ is unsatisfiable; and (iii) it is $\{(0),(1)\}$ if both $\phi$ and $\phi^{\prime}$ are satisfiable. Moreover, clearly, $I=\emptyset$ is partially closed relative to $\left(D_{m}, V\right)$.

(d) Finally, the CQ query $Q$ simply returns $\pi_{Y}(R)$.

We next show that $I=\emptyset$ is in $\operatorname{RCQ}^{w}\left(Q, D_{m}, V\right)$ if and only if $\phi$ is unsatisfiable or $\phi^{\prime}$ is satisfiable. Note that $I=\emptyset$ is in $\operatorname{RCQ}^{w}\left(Q, D_{m}, V\right)$ if and only if $\bigcap_{I^{\prime} \neq \emptyset,\left(I^{\prime}, D_{m}\right) \models V} Q\left(I^{\prime}\right)=\emptyset$. Since $Q$ is monotonic, the latter is equivalent to $\bigcap_{\left(\{t\}, D_{m}\right) \models V} Q(\{t\})=\emptyset$, which happens only when either $\left\{t[y] \mid\left(\{t\}, D_{m}\right) \models V\right\}=\varnothing$, meaning that $\phi$ is not satisfiable, or when $\left\{t[y] \mid\left(\{t\}, D_{m}\right) \models V\right\} \neq \varnothing$ and there exist two elements in this set. In this case, $Q\left(\left\{t_{1}\right\}\right)=\left\{t_{1}\right\} \neq\left\{t_{2}\right\}=Q\left(\left\{t_{2}\right\}\right)$. Hence, the only case in which $I \notin \mathrm{RCQ}^{w}\left(Q, D_{m}, V\right)$ is when $\phi$ is satisfiable and $\phi^{\prime}$ is not satisfiable.

Upper bound. Based on Lemma 5.7, the following algorithm decides whether a given $c$-instance $\mathcal{T}$ is a minimal instance weakly complete for a $\mathrm{CQ}$ query $Q$ relative to $\left(D_{m}, V\right)$ :

(1) Check whether $|\mathcal{T}|>1$. If so, return "no"; otherwise, continue.

(2) Check whether $\emptyset \in \mathrm{RCQ}^{w}\left(Q, D_{m}, V\right)$ and $\mathcal{T}=\emptyset$. If so, return "yes."

(3) Check whether $\emptyset \notin \operatorname{RCQ}^{w}\left(Q, D_{m}, V\right)$ and $|\mathcal{T}|=1$. If so, return "yes"; otherwise, return "no."

It is in NP to check whether $\emptyset \notin \operatorname{RCQ}^{w}\left(Q, D_{m}, V\right) . \emptyset \notin \operatorname{RCQ}^{w}\left(Q, D_{m}, V\right)$ if and only if $\bigcap_{I^{\prime} \in \operatorname{Ext}(\emptyset)} Q\left(I^{\prime}\right) \neq \varnothing$. One can verify that the latter holds if and only if $\bigcap_{\left(\{t\}, D_{m}\right) \models V} Q(\{t\}) \neq$ $\emptyset$. As discussed before, we have that $\emptyset \notin \mathrm{RCQ}^{w}\left(Q, D_{m}, V\right)$ if and only if, for every pair of tuples $t_{1}$ and $t_{2}$ that possibly refer to the same tuple, $Q\left(t_{1}\right)=Q\left(t_{2}\right)$ if $\left(\left\{t_{1}\right\}, D_{m}\right) \models V$ and $\left(\left\{t_{2}\right\}, D_{m}\right) \models V$. Accordingly, we give a coNP algorithm for its complement problem, that is, checking whether $\emptyset \in \operatorname{RCQ}^{w}\left(Q, D_{m}, V\right)$, which returns "no" if and only if there exist a pair of tuples $t_{1}$ and $t_{2}$ such that $Q\left(t_{1}\right) \neq Q\left(t_{2}\right)$ if $\left(\left\{t_{1}\right\}, D_{m}\right) \models V$ and $\left(\left\{t_{2}\right\}, D_{m}\right) \models V$. More specifically, the algorithm works as follows.

(1) Guess one pair of tuples $\left(t_{1}, t_{2}\right)$ with values in Adom.

(2) Check whether $\left(\left\{t_{1}\right\}, D_{m}\right) \models V$ and $\left(\left\{t_{2}\right\}, D_{m}\right) \models V$. If so, continue; otherwise, reject the guess. This can be done in PTIME for one-tuple instances.

(3) Check whether $Q\left(t_{1}\right) \neq Q\left(t_{2}\right)$. If so, return "no"; otherwise, reject the guess. This can be done again in PTIME for one-tuple instances.

It is in coNP to check whether $\emptyset \in \mathrm{RCQ}^{w}\left(Q, D_{m}, V\right)$; thus, it is in NP to check whether $\emptyset \notin \operatorname{RCQ}^{w}\left(Q, D_{m}, V\right)$. Thus, the algorithm for deciding $\operatorname{MINP}^{w}(C Q)$ is in coDP $=\mathrm{NP}$ UcoNP.

In this proof, we consider only those $\mathcal{T}$ such that $\operatorname{Mod}\left(\mathcal{T}, D_{m}, V\right) \neq \emptyset$. However, without assuming $\operatorname{Mod}\left(\mathcal{T}, D_{m}, V\right) \neq \varnothing, \operatorname{MINP}^{w}(\mathrm{CQ})$ is still in coDP. It is in NP to check whether $\operatorname{Mod}\left(\mathcal{T}, D_{m}, V\right) \neq \varnothing$ since $|\mathcal{T}|=1$. That is, adding an extra step to check whether $\operatorname{Mod}\left(\mathcal{T}, D_{m}, V\right) \neq \emptyset$ to the algorithm will not complicate the analysis. 


\section{VIABLE RELATIVE INFORMATION COMPLETENESS}

We next investigate RCDP, RCQP, and MINP for viably complete $c$-instances, denoted by $\mathrm{RCDP}^{v}, \mathrm{RCQP}^{v}$, and MINP ${ }^{v}$, respectively. That is, we now focus on databases that can be made relatively complete when their missing values are correctly instantiated. In this model, we provide complexity results for these problems for various query languages. The results tell us that missing values complicate the analysis of these problems to an extent. As opposed to their counterparts in the weak model, the complexity bounds are not very diverse (we defer the proofs of the results of this section to the electronic appendix). We use $\mathrm{RCQ}^{v}\left(Q, D_{m}, V\right)$ to represent the set of all viably complete instances.

In contrast to Theorem $4.1, \mathrm{RCDP}^{v}(\mathrm{CQ})$ for $c$-instances is $\Sigma_{3}^{p}$-complete rather than $\Pi_{2}^{p}$-complete. Here, $\mathrm{RCDP}^{v}(\mathrm{FP})$ remains undecidable, as opposed to its counterpart in the weak model (Theorem 5.1).

TheOREM 6.1. For c-instances, $\operatorname{RCDP}^{v}\left(\mathcal{L}_{Q}\right)$ is

-undecidable when $\mathcal{L}_{Q}$ is $F O$ or FP, and

$-\Sigma_{3}^{p}$-complete when $\mathcal{L}_{Q}$ is $C Q, U C Q$, or $\exists F^{+}$.

The complexity is unchanged when $D_{m}$ and $V$ are fixed.

In contrast to Theorem 5.1, $\operatorname{RCQP}^{v}\left(\mathcal{L}_{Q}\right)$ is no longer trivial for viably complete $c$ instances when $\mathcal{L}_{Q}$ is FP. One can verify that Lemma 4.4 still holds in this setting. As a result, $\mathrm{RCQP}^{v}$ for relatively viably complete $c$-instances coincides with RCQP for ground instances. For the latter, the complexity results are already established by Theorem 4.5. From these, this corollary follows.

Corollary 6.2. For c-instances, $\operatorname{RCQP}^{v}\left(\mathcal{L}_{Q}\right)$ is

-undecidable when $\mathcal{L}_{Q}$ is $F O$ or FP, and

- NEXPTIME-complete when $\mathcal{L}_{Q}$ is $C Q, U C Q$, or $\exists \mathrm{FO}^{+}$.

The complexity is unchanged when $D_{m}$ and $V$ are fixed.

For $c$-instances, $\operatorname{MINP}^{v}\left(\mathcal{L}_{Q}\right)$ becomes $\Sigma_{3}^{p}$-complete for $\mathrm{CQ}, \mathrm{UCQ}$, or $\exists \mathrm{FO}^{+}$, rather than $\Pi_{3}^{p}$-complete, as in the strong model. The complexity bound is rather robust: it is the same for $\mathrm{CQ}, \mathrm{UCQ}$, and $\exists \mathrm{FO}^{+}$, as opposed to their counterparts in the weak model.

Corollary 6.3. $\operatorname{MINP}^{v}\left(\mathcal{L}_{Q}\right)$ is

- undecidable for c-instances and for ground instances when $\mathcal{L}_{Q}$ is $F O$ or $F P$, and $-\Sigma_{3}^{p}$-complete for c-instances and $\mathrm{D}_{2}^{p}$-complete for ground instances, when $\mathcal{L}_{Q}$ is $C Q$, $U C Q$ or $\exists \mathrm{FO}^{+}$.

The complexity is unchanged when $D_{m}$ and $V$ are fixed.

\section{TRACTABLE SPECIAL CASES}

The results of Sections 4, 5, and 6 tell us that RCDP, RCQP, and MINP have rather high complexity. For practical use to emerge from the study of relative information completeness, we need to develop effective and efficient heuristic algorithms RCDP, RCQP, and MINP, and to identify their special cases that are practical and tractable.

In practice, we often deal with fixed sets of queries and constraints. That is, the queries and CCs are predefined in advance, and only the underlying databases and master data may vary. We often have a fixed query load, for example, in e-commerce, certain fixed Web forms are used, which are fixed queries in which some designated variables may take various value parameters. Moreover, people typically first design constraints based on schemas, then populate and maintain database instances. This 
highlights the need for studying the data complexity of relative information completeness (see Abiteboul et al. [1995] for details about data complexity).

In this section, we identify tractable cases for RCDP, RCQP, and MINP when queries $Q$ and CCs $V$ are fixed, while the underlying databases $D$ and master data $D_{m}$ vary, for $c$-instances in the strong, weak, and viable completeness models. That is, we study their tractable cases under data complexity (the proofs of the results are given in the electronic appendix). In contrast, Sections 4, 5, and 6 have studied the combined complexity of the problems when data, queries, and CCs may all vary.

One might be tempted to think that the data complexity analyses of these problems would be much simpler. The study of data complexity is nontrivial, however. For ground instances in the strong completeness model, the data complexity of RCDP and MINP has recently been studied [Cao et al. 2014]. It is shown that RCDP and MINP remain undecidable for $\mathrm{FO}$ even in the absence of $\mathrm{CCs}$, and for FP when $V$ is a set of FDs. These undecidability results obviously carry over to $c$-instances in the strong model. While these problems are in PTIME for $\mathrm{CQ}, \mathrm{UCQ}$, and $\exists \mathrm{FO}^{+}$when ground instances are considered, the PTIME algorithms of Cao et al. [2014] no longer work on $c$-instances. To the best of our knowledge, no previous work has studied RCQP for ground instances or $c$-instances, or RCDP and MINP for $c$-instances.

The relatively complete database problem. To get tractable cases for $c$-instances, we consider $c$-instances with a constant number of variables, that is, when our databases have a small number of missing (null) values. Under this condition and data complexity, RCDP becomes tractable for most positive query languages.

CoROLlary 7.1. For c-instances with a constant number of variables, and for fixed query $Q$ and a fixed set $V$ of $C C s$,

$-\mathrm{RCDP}^{s}$ and $\mathrm{RCDP}^{v}$ are in PTIME for $C Q, U C Q$, and $\exists \mathrm{FO}^{+}$; and $-\mathrm{RCDP}^{w}$ is in PTIME for CQ, UCQ, $\exists F O^{+}$, and $F P$.

The relatively complete query problem. When we use INDs as CCs, that is, for $\mathrm{CCs}$ of the form $q \subseteq p$ when $q$ and $p$ are both projection queries, $\mathrm{RCQP}^{s}$ and $\mathrm{RCQP}^{v}$ become much simpler. The positive results hold even when the set $V$ of CCs is not fixed. Moreover, RCDP ${ }^{w}$ is in constant time for FP for general CCs defined in CQ, by Theorem 5.4.

Corollary 7.2. For fixed queries,

$-\mathrm{RCQP}^{s}$ and $\mathrm{RCQP}^{v}$ are in PTIME for CQ, UCQ, and $\exists F O^{+}$when CCs are INDs; and $-\mathrm{RCQP}^{w}$ is in $O(1)$ time for $C Q, U C Q, \exists F O^{+}$, and $F P$.

The minimality problem. Similar to RCDP, we get tractable cases of MINP, when queries and CCs are fixed, for $c$-instances with a constant number of variables.

Corollary 7.3. For c-instances with a constant number of variables, and for fixed query $Q$ and a fixed set $V$ of CCs,

- MINP ${ }^{s}$ and $\mathrm{MINP}^{v}$ are in PTIME for $C Q, U C Q$, and $\exists \mathrm{FO}^{+}$; and

$-\mathrm{MINP}^{w}$ is in PTIME for CQ.

\section{CONCLUSIONS}

We have proposed three models to specify the relative information completeness of databases in the presence of both missing values and missing tuples. We have studied the interaction between the analysis of relative completeness and the analysis of data consistency. We have also identified three problems associated with relative 
completeness: RCQP, RCDP, and MINP. For a variety of query languages, we have established upper and lower bounds on these problems, all matching, in each of the three completeness models, both for $c$-instances and for ground instances. We have also identified tractable cases of these problems under data complexity. We expect that these results will help database users decide whether their queries can find complete answers in a database, and will help developers of MDM or databases identify a minimal amount of information to collect in order to answer queries commonly issued.

The main complexity results are summarized in Table I, annotated with their corresponding theorems. From the table we can see that different combinations of query languages, completeness models, and the presence and the absence of missing values lead to a spectrum of decision problems with different complexity bounds.

The study of relative information completeness is still in its infancy. An open issue concerns the complexity of RCQP for FO in the weak model. We only know that it is undecidable for ground instances; our conjecture is that it is also undecidable for $c$-instances. Another open issue concerns whether the complexity bounds remain intact when master data and CCs are fixed. A third topic is to develop representation systems for relatively complete databases, possibly under the semantics introduced by Libkin [2014]. A fourth topic is to figure out the impact of other constraints on the analysis of relative completeness, such as tuple-generating dependencies. Finally, to make practical use of the study, we need to develop efficient heuristic algorithms for the problems with certain performance guarantees.

\section{ELECTRONIC APPENDIX}

The electronic appendix for this article can be accessed in the ACM Digital Library

\section{REFERENCES}

Serge Abiteboul and Oliver M. Duschka. 1998. Complexity of answering queries using materialized views. In Proceedings of the 17th ACM SIGACT-SIGMOD-SIGART Symposium on Principles of Database Systems (PODS'98). ACM, New York, NY, 254-263. DOI : http://dx.doi.org/10.1145/275487.275516

Serge Abiteboul, Richard Hull, and Victor Vianu. 1995. Foundations of Databases. Addison-Wesley, New York, NY.

Serge Abiteboul, Paris C. Kanellakis, and Gösta Grahne. 1991. On the representation and querying of sets of possible worlds. Theoretical Computer Science 78, 1, 159-187. DOI : http://dx.doi.org/10.1016/0304 $-3975(51) 90007-2$

Marcelo Arenas, Leopoldo Bertossi, and Jan Chomicki. 1999. Consistent query answers in inconsistent databases. In Proceedings of the 18th ACM SIGMOD-SIGACT-SIGART Symposium on Principles of Database Systems (PODS'99). ACM, New York, NY, 68-79. DOI : http://dx.doi.org/10.1145/303976.303983

Marcelo Arenas, Jorge Pérez, Juan L. Reutter, and Cristian Riveros. 2009. Composition and inversion of schema mappings. SIGMOD Record 38, 3, 17-28. DOI : http://dx.doi.org/10.1145/1815933.1815938

Yang Cao, Ting Deng, Wenfei Fan, and Floris Geerts. 2014. On the data complexity of relative information completeness. Information Systems 45, 18-34. DOI : http://dx.doi.org/10.1016/j.is.2014.04.001

Jan Chomicki. 2007. Consistent query answering: Five easy pieces. In Proceedings of the 11th International Conference on Database Theory (ICDT'07). Springer-Verlag, New York, NY, 1-17. DOI:http:// dx.doi.org/10.1007/11965893_1

Charles Elkan. 1990. Independence of logic database queries and update. In Proceedings of the 9th ACM SIGACT-SIGMOD-SIGART Symposium on Principles of Database Systems. ACM, New York, NY, 154 160. DOI : http://dx.doi.org/10.1145/298514.298557

Wenfei Fan. 2008. Dependencies revisited for improving data quality. In Proceedings of the 27th ACM SIGMOD-SIGACT-SIGART Symposium on Principles of Database Systems (PODS'08). ACM, New York, NY, 159-170. DOI : http://dx.doi.org/10.1145/1376916.1376940

Wenfei Fan and Floris Geerts. 2009. Relative information completeness. In Proceedings of the 28th ACM SIGMOD-SIGACT-SIGART Symposium on Principles of Database Systems (PODS'09). ACM, New York, NY, 97-106. DOI : http://dx.doi.org/10.1145/1559795.1559811 
Wenfei Fan and Floris Geerts. 2010a. Capturing missing tuples and missing values. In Proceedings of the 29th ACM SIGMOD-SIGACT-SIGART Symposium on Principles of Database Systems (PODS'10). ACM, New York, NY, 169-178. DOI : http://dx.doi.org/10.1145/1807085.1807109

Wenfei Fan and Floris Geerts. 2010b. Relative information completeness. ACM Transactions on Database Systems 35, 4, Article 27, 44 pages. DOI : http://dx.doi.org/10.1145/1862919.1862924

Wenfei Fan and Floris Geerts. 2012. Foundations of Data Quality Management. Morgan \& Claypool Publishers, San Francisco, CA. DOI : http://dx.doi.org/10.2200/S00439ED1V01Y201207DTM030

Georg Gottlob and Roberto Zicari. 1988. Closed world databases opened through null values. In Proceedings of the 14th International Conference on Very Large Data Bases (VLDB'88). Morgan Kaufmann Publishers Inc., San Francisco, CA, 50-61. http://dl.acm.org/citation.cfm?id=645915.671794

Gösta Grahne. 1991. The Problem of Incomplete Information in Relational Databases. Lecture Notes in Computer Science, Vol. 554. Springer. DOI : http://dx.doi.org/10.1007/3-540-54919-6

Tomasz Imieliński and Witold Lipski, Jr. 1984. Incomplete information in relational databases. Journal of the ACM 31, 4, 761-791. DOI : http://dx.doi.org/10.1145/1634.1886

Phokion G. Kolaitis. 2005. Schema mappings, data exchange, and metadata management. In Proceedings of the 24th ACM SIGMOD-SIGACT-SIGART Symposium on Principles of Database Systems (PODS'05). ACM, New York, NY, 61-75. DOI : http://dx.doi.org/10.1145/1065167.1065176

Willis Lang, Rimma V. Nehme, Eric Robinson, and Jeffrey F. Naughton. 2014. Partial results in database systems. In Proceedings of the 2014 ACM SIGMOD International Conference on Management of Data (SIGMOD'14). ACM, New York, NY, 1275-1286. DOI : http://dx.doi.org/10.1145/2588555.2612176

Maurizio Lenzerini. 2002. Data integration: A theoretical perspective. In Proceedings of the 21st ACM SIGMOD-SIGACT-SIGART Symposium on Principles of Database Systems (PODS'O2). ACM, New York, NY, 233-246. DOI : http://dx.doi.org/10.1145/543613.543644

Alon Levy, Inderpal Singh Mumick, Yehoshua Sagiv, and Oded Shmueli. 1993. Equivalence, queryreachability and satisfiability in datalog extensions. In Proceedings of the 12th ACM SIGACT-SIGMODSIGART Symposium on Principles of Database Systems (PODS'93). ACM, New York, NY, 109-122. DOI : http://dx.doi.org/10.1145/153850.153860

Alon Y. Levy. 1996. Obtaining complete answers from incomplete databases. In Proceedings of the 22nd International Conference on Very Large Data Bases (VLDB'96). Morgan Kaufmann Publishers Inc., San Francisco, CA, 402-412. http://dl.acm.org/citation.cfm?id=645922.673332

Alon Y. Levy and Yehoshua Sagiv. 1993. Queries independent of updates. In Proceedings of the 19th International Conference on Very Large Data Bases (VLDB'93). Morgan Kaufmann Publishers Inc., San Francisco, CA, 171-181. http://dl.acm.org/citation.cfm?id=645919.672674

Leonid Libkin. 2014. Incomplete data: What went wrong, and how to fix it. In Proceedings of the 33rd ACM SIGMOD-SIGACT-SIGART Symposium on Principles of Database Systems (PODS'14). ACM, New York, NY, 1-13. DOI : http://dx.doi.org/10.1145/2594538.2594561

David Loshin. 2008. Master Data Management. Morgan Kaufmann Publishers Inc., San Francisco, CA.

Microsoft. 2008. SQL Server 2008 R2 Master Data Services. Retrieved April 3, 2016 from http://www. microsoft.com/sqlserver/2008/en/us/MDS.aspx.

Donald W. Miller Jr., John D. Yeast, and Robin L. Evans. 2005. Missing prenatal records at a birth center: A communication problem quantified. In AMIA Annual Symposium Proceedings (AMIA'05). 535-539.

Amihai Motro. 1989. Integrity = validity + completeness. ACM Transactions on Database Systems 14, 4, 480-502. DOI : http://dx.doi.org/10.1145/76902.76904

Dan Olteanu, Christoph Koch, and Lyublena Antova. 2008. World-set decompositions: Expressiveness and efficient algorithms. Theoretical Computer Science 403, 2-3, 265-284.

Christos H. Papadimitriou. 1994. Computational Complexity. Addison-Wesley, New York, NY.

John Radcliffe and Andrew White. 2008. Key issues for Master Data Management. Gartner. Retrieved April 3, 2016 from https://www.gartner.com/doc/590507/key-issues-master-data-management.

Luc Segoufin and Victor Vianu. 2005. Views and queries: Determinacy and rewriting. In Proceedings of the 24th ACM SIGMOD-SIGACT-SIGART Symposium on Principles of Database Systems (PODS'05). ACM, New York, NY, 49-60. DOI : http://dx.doi.org/10.1145/1065167.1065174

Mark Spielmann. 2000. Abstract State Machines: Verification Problems and Complexity. Ph.D. Dissertation. RWTH Aachen University, Aachen, Germany.

Boris Trakhtenbrot. 1950. The impossibility of an algorithm for the decidability problem on finite classes. Doklady Akademii Nauk SSSR 70, 4, 569-572.

Ron van der Meyden. 1998. Logical approaches to incomplete information: A survey. In Logics for Databases and Information Systems, J. Chomicki and G. Saake (Eds.). Kluwer, 307-356. 
Moshe Vardi. 1986. On the integrity of databases with incomplete information. In Proceedings of the 5th ACM SIGACT-SIGMOD Symposium on Principles of Database Systems (PODS'86). ACM, New York, NY, 252-266. DOI : http://dx.doi.org/10.1145/6012.15419

Michael Wooldridge and Paul E. Dunne. 2004. On the computational complexity of qualitative coalitional games. Artificial Intelligence 158, 1, 27-73. DOI : http://dx.doi.org/10.1016/j.artint.2004.04.002

Received March 2015; revised October 2015; accepted December 2015 